Supporting Information for

\title{
Is Aromaticity a Driving Force in Catalytic Cycles? The Case from the Cycloisomerization of Enynes Catalyzed by All-Metal Aromatic $\mathrm{Pd}_{3}{ }^{+}$Clusters and Carboxylic Acids
}

\author{
Franca Bigi, ${ }^{a b}$ Gianpiero Cera,${ }^{a}$ Raimondo Maggi, ${ }^{,}$Yanlan Wang, ${ }^{c}$ \\ Max Malacria, ${ }^{,}$Giovanni Maestri ${ }^{*}$ \\ giovanni.maestri@unipr.it
}

a Università di Parma, Department of Chemistry, Life Sciences and Environmental Sustainability, Parco Area delle Scienze 17/A, 43124 Parma, Italy

b IMEM-CNR, Parco Area delle Scienze 37/A, 43124 Parma, Italy

c Liaocheng University, Department of chemistry and chemical engineering, 252059 Liaocheng, China

d Sorbonne Université, Faculty of Science and Engineering, CNRS, Institut Parisien de Chimie Moléculaire (UMR CNRS 8232), Paris 75252 Cedex 05, France 
Table of contents

Comparison of different models

S3

Selected calculated charges on key intermediates

XYZ coordinates

S5

Comprehensive table in A.U. 


\section{Comparison of different models}

The following table compares key interatomic distances from the X-Ray structure of a tripalladium complex used in catalytic reactions and those calculated using different functionals/basis sets. The structure of complex $\mathbf{I}_{\mathbf{A}}, \mathrm{Pd}_{3}(\mathrm{SMe})_{3}(\mathrm{PTol})_{3} \mathrm{SbF}_{6}$, can be freely recovered from the CCDC ( 1017253 ). It is worth noting that to keep the computational cost in check, the study on the mechanism of the catalytic reaction has been carried out with smaller $\mathrm{PMe}_{3}$ phosphines as ligands. On the contrary, the following table compares the results obtained with untruncated cations. While all of the tested models are appropriate to describe the Pd-P and $\mathrm{Pd}-\mathrm{S}$ distances compared to experimental values, the closest match for crucial Pd-Pd ones has been achieved employing the M06 functional. At this level, small differences were observed varying the basis set, and the best correlation is found employing the Def2-SVP one. The XRay structure has $C_{3}$ symmetry, while all calculations were performed without any symmetry constraint, although they nonetheless converged to quasi-symmetric structures.

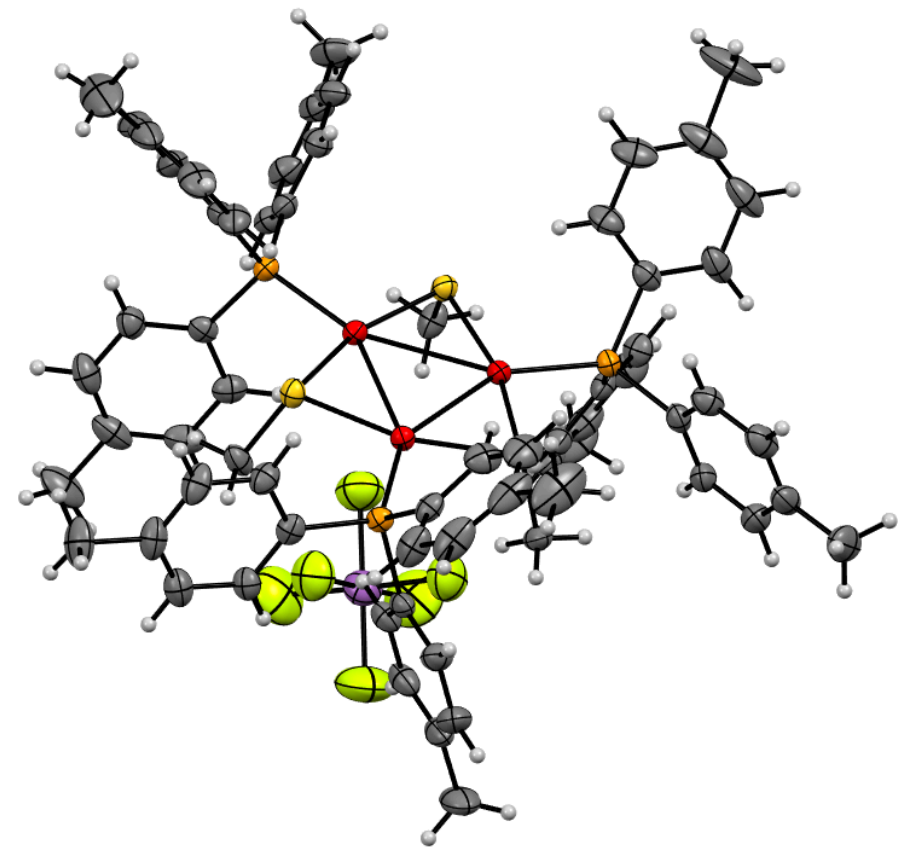

Figure S1. The X-Ray structure of complex $\mathbf{I}_{\mathrm{A}}$

\begin{tabular}{|c|c|c|c|c|c|c|}
\hline & $\begin{array}{c}\text { X- } \\
\text { Ray }\end{array}$ & $\begin{array}{c}\text { M06/Def2- } \\
\text { SVP }\end{array}$ & $\begin{array}{c}\text { M06/lacvp(d } \\
\text { ) }\end{array}$ & $\begin{array}{c}\text { B3LYP } \\
/ \operatorname{lacvp(d)}\end{array}$ & $\begin{array}{c}\text { PBE1PBE } \\
/ \operatorname{lacvp(d)}\end{array}$ & $\begin{array}{c}\text { BP86/lacvp } \\
\text { (d) }\end{array}$ \\
\hline Pd-Pd & 2.84 & $2.93(\mathrm{x} 3)$ & $2.95(\mathrm{x} 3)$ & $3.00(\mathrm{x} 3)$ & $2.97(\mathrm{x} 3)$ & $3.00(\mathrm{x} 3)$ \\
\hline Pd-P & 2.26 & $2.34(\mathrm{x} 3)$ & $2.35(\mathrm{x} 3)$ & $2.37(\mathrm{x} 3)$ & $2.34(\mathrm{x} 3)$ & $2.37(\mathrm{x} 3)$ \\
\hline Pd-S & 2.29 & $2.34(\mathrm{x} 6)$ & $2.35(\mathrm{x} 3) /$ & $2.35(\mathrm{x} 3) /$ & $2.34(\mathrm{x} 3) /$ & $2.35(\mathrm{x} 3) /$ \\
& & & $2.34(\mathrm{x} 3)$ & $2.34(\mathrm{x} 3)$ & $2.33(\mathrm{x} 3)$ & $2.34(\mathrm{x} 3)$ \\
\hline
\end{tabular}

Table T1. Comparison of key distances, values in $\AA$. 


\section{Selected calculated charges on key intermediates}

According to Mulliken charges, the Pd atoms bound to the hydride nucleus are more electronrich among intermediates IV-VI, in agreement with the strong donating influence of the latter. This might correlate with a higher reactivity with respect to the dissociation of a phosphorous ligands and subsequent substrate coordination, which then initiate the catalytic cycloisomerization. This trend is less manifest observing APT charges, in particular for bridged hydride IV.

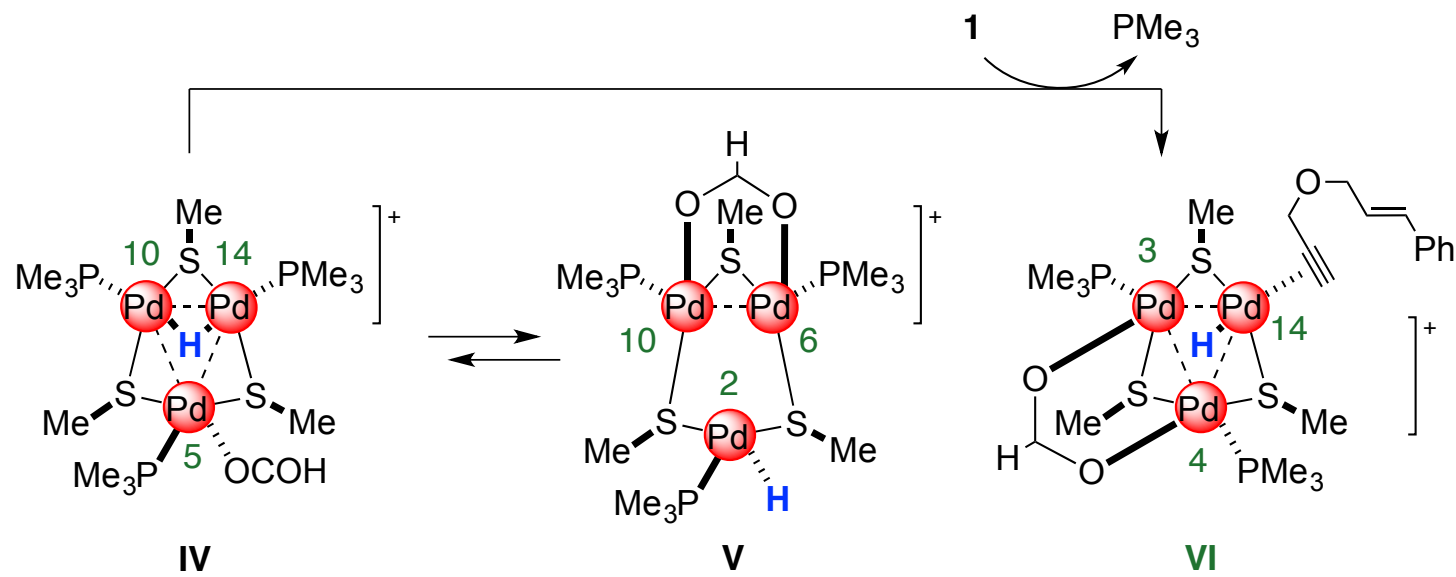

\begin{tabular}{|l|l|l|l|l|l|l|}
\hline & IV & & V & & VI & \\
\hline Mulliken & $\operatorname{Pd}(5)$ & +0.29 & $\operatorname{Pd}(2)$ & -0.03 & $\operatorname{Pd}(3)$ & +0.13 \\
\hline & $\operatorname{Pd}(10)$ & -0.27 & $\operatorname{Pd}(6)$ & +0.01 & $\operatorname{Pd}(4)$ & -0.05 \\
\hline & $\operatorname{Pd}(14)$ & -0.29 & $\operatorname{Pd}(10)$ & +0.12 & $\operatorname{Pd}(14)$ & -0.09 \\
\hline & $\mathrm{H}$ & +0.16 & $\mathrm{H}$ & -0.06 & $\mathrm{H}$ & -0.05 \\
\hline APT & $\mathrm{Pd}(5)$ & +0.16 & $\mathrm{Pd}(2)$ & -0.15 & $\mathrm{Pd}(3)$ & +0.26 \\
\hline & $\mathrm{Pd}(10)$ & +0.14 & $\mathrm{Pd}(6)$ & +0.26 & $\operatorname{Pd}(4)$ & +0.33 \\
\hline & $\mathrm{Pd}(14)$ & +0.12 & $\mathrm{Pd}(10)$ & +0.20 & $\operatorname{Pd}(14)$ & +0.11 \\
\hline & $\mathrm{H}$ & -0.09 & $\mathrm{H}$ & -0.02 & $\mathrm{H}$ & -0.01 \\
\hline
\end{tabular}

Table T2. Comparison of Mulliken (up) and APT (down) charges on Pd atoms and the hydride nucleus of intermediates IV, V and VI. 
XYZ coordinates

\section{Def2-SVP (gas)}

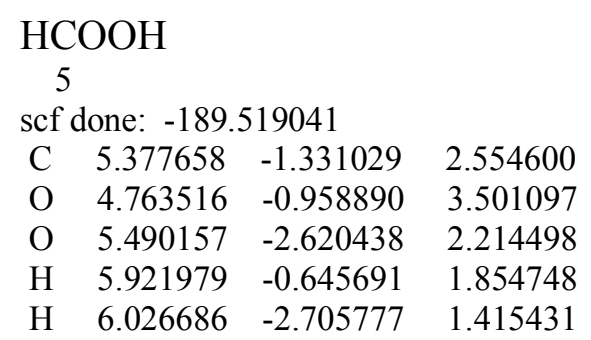

$\mathrm{PMe}_{3}$

13

scf done: -460.776896

$\begin{array}{llll}\text { C } & 2.317406 & 5.823480 & 3.089501\end{array}$

$\begin{array}{llll}\mathrm{P} & 2.590726 & 5.578512 & 1.272480\end{array}$

$\begin{array}{llll}\text { C } & 1.093619 & 6.478294 & 0.652169\end{array}$

$\begin{array}{llll}\text { C } & 3.845232 & 6.916395 & 1.002794\end{array}$

$\begin{array}{llll}\mathrm{H} & 3.520366 & 7.884471 & 1.424365\end{array}$

$\begin{array}{llll}\mathrm{H} & 4.801888 & 6.630970 & 1.469492\end{array}$

$\begin{array}{llll}\mathrm{H} & 4.027727 & 7.044916 & -0.076293\end{array}$

$\begin{array}{llll}\mathrm{H} & 2.113135 & 6.879474 & 3.342103\end{array}$

$\begin{array}{llll}\mathrm{H} & 1.465964 & 5.209128 & 3.424040\end{array}$

H $\quad 3.206335 \quad 5.492125 \quad 3.650141$

$\begin{array}{llll}\mathrm{H} & 0.992571 & 7.482245 & 1.102135\end{array}$

$\begin{array}{llll}\mathrm{H} & 1.148901 & 6.585942 & -0.443007\end{array}$

$\begin{array}{llll}\mathrm{H} & 0.187355 & 5.895166 & 0.882571\end{array}$

\section{Enyne 1}

$$
25
$$

scf done: -538.816233

$\begin{array}{rrrr}\mathrm{C} & 1.506825 & 3.892920 & -2.396851 \\ \mathrm{C} & 2.182382 & 3.087599 & -3.328459 \\ \mathrm{C} & 3.406747 & 3.547631 & -3.834458 \\ \mathrm{C} & 3.934481 & 4.773133 & -3.439455 \\ \mathrm{C} & 3.248168 & 5.563840 & -2.521326 \\ \mathrm{C} & 2.033211 & 5.116009 & -2.000497 \\ \mathrm{C} & 1.669678 & 1.789358 & -3.767935 \\ \mathrm{C} & 0.461447 & 1.271861 & -3.510602 \\ \mathrm{C} & 0.033208 & -0.088457 & -3.943742 \\ \mathrm{O} & -0.402072 & -0.903118 & -2.879299 \\ \mathrm{C} & 0.574919 & -1.167812 & -1.912701 \\ \mathrm{C} & 0.732085 & -0.123357 & -0.898756 \\ \mathrm{C} & 0.870634 & 0.717650 & -0.038595 \\ \mathrm{H} & 0.996465 & 1.467448 & 0.720317 \\ \mathrm{H} & -0.829219 & -0.030104 & -4.630475 \\ \mathrm{H} & 0.861740 & -0.583153 & -4.496931 \\ \mathrm{H} & 1.562062 & -1.359314 & -2.387448 \\ \mathrm{H} & 0.282958 & -2.106952 & -1.410748 \\ \mathrm{H} & -0.277007 & 1.834882 & -2.924793 \\ \mathrm{H} & 2.372350 & 1.186638 & -4.362994 \\ \mathrm{H} & 3.949304 & 2.925357 & -4.554539 \\ \mathrm{H} & 4.890045 & 5.112877 & -3.849781 \\ \mathrm{H} & 3.660232 & 6.526632 & -2.205538\end{array}$



H $\quad 1.492345 \quad 5.727620 \quad-1.272219$
H $\quad 0.561019 \quad 3.546567 \quad-1.968478$

\section{Diene 3}

25

scf done: -538.877005

$\begin{array}{lrrr}\text { C } & -3.508250 & 2.444673 & -1.786972 \\ \mathrm{C} & -3.936309 & 2.953485 & -0.546862 \\ \mathrm{C} & -4.716051 & 4.124664 & -0.554637 \\ \mathrm{C} & -5.028417 & 4.778380 & -1.739322 \\ \mathrm{C} & -4.579669 & 4.269271 & -2.957166 \\ \mathrm{C} & -3.826035 & 3.097136 & -2.973488 \\ \mathrm{C} & -3.630386 & 2.338474 & 0.738578 \\ \mathrm{C} & -2.686102 & 1.427968 & 1.049458 \\ \mathrm{C} & -1.642015 & 0.805014 & 0.159826 \\ \mathrm{O} & -0.668146 & 0.274822 & 1.020971 \\ \mathrm{C} & -1.296446 & -0.090185 & 2.222191 \\ \mathrm{C} & -2.444068 & 0.871404 & 2.389227 \\ \mathrm{C} & -3.083283 & 1.133826 & 3.534354 \\ \mathrm{H} & -4.247188 & 2.702893 & 1.571319 \\ \mathrm{H} & -3.922234 & 1.834787 & 3.584221 \\ \mathrm{H} & -2.785646 & 0.651661 & 4.470554 \\ \mathrm{H} & -0.564295 & -0.045337 & 3.045019 \\ \mathrm{H} & -1.679285 & -1.134557 & 2.173065 \\ \mathrm{H} & -2.091274 & 0.004735 & -0.473546 \\ \mathrm{H} & -1.164754 & 1.530896 & -0.522095 \\ \mathrm{H} & -5.072203 & 4.526497 & 0.400115 \\ \mathrm{H} & -5.630020 & 5.691688 & -1.714556 \\ \mathrm{H} & -4.825324 & 4.779600 & -3.892774 \\ \mathrm{H} & -3.485538 & 2.679335 & -3.925551 \\ \mathrm{H} & -2.946090 & 1.509028 & -1.832743\end{array}$

A

57

scf done: -3079.910710

$\begin{array}{llll}\text { C } & 7.242980 & 2.010922 & -2.016007\end{array}$

$\begin{array}{llll}\text { P } & 7.079758 & 0.868051 & -0.602692\end{array}$

$\begin{array}{llll}\text { C } & 7.175829 & -0.785258 & -1.373631\end{array}$

$\begin{array}{llll}\mathrm{Pd} & 8.668888 & 1.438084 & 0.990999\end{array}$

$\begin{array}{llll}\text { Pd } & 11.434511 & 1.424680 & 1.893398\end{array}$

$\begin{array}{llll}\mathrm{P} & 13.642334 & 0.818286 & 1.506327\end{array}$

$\begin{array}{llll}\text { C } & 14.853939 & 1.057898 & 2.852720\end{array}$

$\begin{array}{llll}\text { C } & 5.316702 & 1.008137 & -0.144817\end{array}$

$\begin{array}{llll}\text { S } & 10.457166 & 0.165019 & 0.175124\end{array}$

$\begin{array}{llll}\text { C } & 10.201908 & -1.480601 & 0.939058\end{array}$

$\begin{array}{llll}\mathrm{Pd} & 9.501688 & 3.200178 & 3.157661\end{array}$

$\begin{array}{llll}S & 11.788446 & 2.984731 & 3.601422\end{array}$

$\begin{array}{llll}\text { C } & 11.849720 & 1.964034 & 5.120258\end{array}$

$\begin{array}{llll}\text { S } & 7.390065 & 3.013239 & 2.159716\end{array}$

$\begin{array}{llll}\text { C } & 6.423228 & 1.994068 & 3.334602\end{array}$

$\begin{array}{llll}\mathrm{P} & 9.042260 & 5.019795 & 4.522927\end{array}$

$\begin{array}{llll}\text { C } & 13.986170 & -0.886769 & 0.948310\end{array}$

$\begin{array}{llll}\text { C } & 14.292405 & 1.850674 & 0.149180\end{array}$

$\mathrm{H} \quad 11.649832 \quad 2.608980 \quad 5.989221$

H $\quad 12.862672 \quad 1.547164 \quad 5.227200$

H $11.115713 \quad 1.147361 \quad 5.090100$

$\begin{array}{llll}\mathrm{H} & 5.587523 & 1.526059 & 2.792638\end{array}$

H $6.003839 \quad 2.646833 \quad 4.114945$ 


$\begin{array}{cccc}\text { H } & 7.043028 & 1.214511 & 3.798315 \\ \mathrm{H} & 11.137731 & -2.055544 & 0.872942 \\ \mathrm{H} & 9.427594 & -2.019239 & 0.372664 \\ \mathrm{H} & 9.895826 & -1.395371 & 1.990657 \\ \mathrm{H} & 15.060686 & -1.010756 & 0.732914 \\ \mathrm{H} & 13.694873 & -1.606238 & 1.729298 \\ \mathrm{H} & 13.408154 & -1.101785 & 0.035919 \\ \mathrm{H} & 15.872761 & 0.822797 & 2.501651 \\ \mathrm{H} & 14.821797 & 2.102381 & 3.200193 \\ \mathrm{H} & 14.609791 & 0.397348 & 3.699216 \\ \mathrm{H} & 15.342969 & 1.599348 & -0.073665 \\ \mathrm{H} & 13.678395 & 1.692900 & -0.751464 \\ \mathrm{H} & 14.223299 & 2.912921 & 0.430624 \\ \mathrm{H} & 4.673212 & 0.749784 & -1.002465 \\ \mathrm{H} & 5.085141 & 0.328232 & 0.690096 \\ \mathrm{H} & 5.100012 & 2.041346 & 0.169274 \\ \mathrm{H} & 7.011326 & -1.567702 & -0.616569 \\ \mathrm{H} & 6.409849 & -0.887508 & -2.160564 \\ \mathrm{H} & 8.172120 & -0.927674 & -1.821146 \\ \mathrm{H} & 7.112225 & 3.045801 & -1.662684 \\ \mathrm{H} & 8.253045 & 1.918884 & -2.445200 \\ \mathrm{H} & 6.492776 & 1.791459 & -2.793988 \\ \mathrm{C} & 10.121299 & 5.315306 & 5.967426 \\ \mathrm{C} & 9.213815 & 6.546044 & 3.537712 \\ \mathrm{C} & 7.364968 & 5.159639 & 5.234363 \\ \mathrm{H} & 7.264251 & 6.104498 & 5.794410 \\ \mathrm{H} & 6.617082 & 5.140087 & 4.426497 \\ \mathrm{H} & 7.170438 & 4.318935 & 5.918386 \\ \mathrm{H} & 8.996769 & 7.438356 & 4.148513 \\ \mathrm{H} & 10.239358 & 6.613522 & 3.142532 \\ \mathrm{H} & 8.517887 & 6.506953 & 2.685024 \\ \mathrm{H} & 9.836653 & 6.252645 & 6.474172 \\ \mathrm{H} & 10.029834 & 4.483688 & 6.683264 \\ \mathrm{H} & 11.170567 & 5.385214 & 5.640757\end{array}$

\section{I}

62

scf done: -3269.449024

$\begin{array}{cccc}\text { C } & 7.945801 & 1.798463 & -2.137074 \\ \mathrm{P} & 7.116391 & 1.401854 & -0.562796 \\ \mathrm{Pd} & 6.805679 & -0.902021 & -0.413027 \\ \mathrm{~S} & 4.799609 & -1.036213 & -1.621425 \\ \mathrm{C} & 3.423888 & -0.559873 & -0.512169 \\ \mathrm{C} & 8.161197 & 2.196925 & 0.706208 \\ \mathrm{C} & 5.622790 & 2.451897 & -0.573097 \\ \mathrm{Pd} & 7.813761 & -3.541430 & 0.281279 \\ \mathrm{~S} & 6.311095 & -5.220181 & -0.338754 \\ \mathrm{C} & 5.423996 & -5.693530 & 1.191526 \\ \mathrm{~S} & 8.879719 & -1.468693 & 0.524437 \\ \mathrm{C} & 8.815305 & -1.100480 & 2.315872 \\ \mathrm{Pd} & 5.257187 & -3.271881 & -1.083888 \\ \mathrm{P} & 3.341086 & -4.237030 & -1.980687 \\ \mathrm{C} & 1.910529 & -4.180082 & -0.845951 \\ \mathrm{P} & 9.353846 & -4.873835 & 1.398215 \\ \mathrm{C} & 2.723367 & -3.459089 & -3.511391 \\ \mathrm{C} & 3.469738 & -6.000520 & -2.433068 \\ \mathrm{O} & 5.388775 & -2.409464 & 2.357370 \\ \mathrm{C} & 5.208172 & -1.141112 & 2.749374 \\ \mathrm{O} & 4.703004 & -0.835747 & 3.780559\end{array}$




\begin{tabular}{cccc}
$\mathrm{H}$ & 6.024984 & -6.445687 & 1.726500 \\
$\mathrm{H}$ & 4.469944 & -6.165166 & 0.909496 \\
$\mathrm{H}$ & 5.231426 & -4.833719 & 1.849136 \\
$\mathrm{H}$ & 8.730508 & -0.012736 & 2.458329 \\
$\mathrm{H}$ & 9.761148 & -1.429342 & 2.773807 \\
$\mathrm{H}$ & 7.972729 & -1.601217 & 2.814073 \\
$\mathrm{H}$ & 2.476408 & -0.686945 & -1.058811 \\
$\mathrm{H}$ & 3.523380 & 0.503227 & -0.246023 \\
$\mathrm{H}$ & 3.398867 & -1.166012 & 0.404747 \\
$\mathrm{H}$ & 1.030503 & -4.661601 & -1.304683 \\
$\mathrm{H}$ & 2.154391 & -4.696436 & 0.095630 \\
$\mathrm{H}$ & 1.663307 & -3.134276 & -0.607020 \\
$\mathrm{H}$ & 2.524428 & -6.354887 & -2.876961 \\
$\mathrm{H}$ & 4.286883 & -6.136508 & -3.157871 \\
$\mathrm{H}$ & 3.701022 & -6.605059 & -1.542890 \\
$\mathrm{H}$ & 1.829912 & -3.988826 & -3.881753 \\
$\mathrm{H}$ & 2.473407 & -2.403681 & -3.324323 \\
$\mathrm{H}$ & 3.509423 & -3.489628 & -4.281403 \\
$\mathrm{H}$ & 8.270980 & 3.273664 & 0.494640 \\
$\mathrm{H}$ & 7.702355 & 2.079829 & 1.701507 \\
$\mathrm{H}$ & 9.158514 & 1.729930 & 0.715350 \\
$\mathrm{H}$ & 5.064943 & 2.325906 & 0.368926 \\
$\mathrm{H}$ & 5.899824 & 3.514232 & -0.678243 \\
$\mathrm{H}$ & 4.972755 & 2.167789 & -1.415536 \\
$\mathrm{H}$ & 8.914241 & 1.276127 & -2.182054 \\
$\mathrm{H}$ & 7.323650 & 1.443686 & -2.973888 \\
$\mathrm{H}$ & 8.108846 & 2.884528 & -2.235819 \\
$\mathrm{H}$ & 5.594158 & -0.403645 & 1.989043 \\
$\mathrm{H}$ & 5.815510 & -2.432789 & 1.469851 \\
$\mathrm{C}$ & 9.422708 & -6.626663 & 0.895878 \\
$\mathrm{C}$ & 11.097693 & -4.344143 & 1.304747 \\
$\mathrm{C}$ & 8.996558 & -4.937596 & 3.188311 \\
$\mathrm{H}$ & 9.698133 & -5.612445 & 3.706614 \\
$\mathrm{H}$ & 9.081286 & -3.929371 & 3.622213 \\
$\mathrm{H}$ & 7.965923 & -5.290111 & 3.350657 \\
$\mathrm{H}$ & 11.745635 & -5.041508 & 1.861404 \\
$\mathrm{H}$ & 11.416093 & -4.313512 & 0.251504 \\
$\mathrm{H}$ & 11.207469 & -3.332030 & 1.722906 \\
$\mathrm{H}$ & 10.190709 & -7.165384 & 1.475376 \\
$\mathrm{H}$ & 8.444227 & -7.104366 & 1.057032 \\
$\mathrm{H}$ & 9.660733 & -6.694087 & -0.176708 \\
& & & \\
\hline
\end{tabular}

\section{TS (I-IV)}

62

scf done: -3269.412742

$\begin{array}{cccc}\mathrm{C} & 0.456752 & 0.885126 & -0.399768 \\ \mathrm{P} & 0.326955 & 0.695711 & 1.409883 \\ \mathrm{C} & 2.021801 & 0.964505 & 2.018001 \\ \mathrm{Pd} & -1.169594 & 2.116523 & 2.403678 \\ \mathrm{~S} & -2.487385 & 0.301900 & 3.147031 \\ \mathrm{C} & -3.486594 & 0.931276 & 4.540999 \\ \mathrm{~S} & -0.332480 & 3.845712 & 1.021994 \\ \mathrm{C} & -0.678353 & 5.408167 & 1.900056 \\ \mathrm{Pd} & -2.438116 & 3.263302 & -0.111168 \\ \mathrm{~S} & -4.348032 & 2.119025 & -0.845194 \\ \mathrm{C} & -5.750640 & 3.180282 & -0.341279 \\ \mathrm{P} & -1.916760 & 4.564469 & -1.995217 \\ \mathrm{C} & -0.737752 & 3.723449 & -3.108915 \\ \mathrm{C} & -3.318983 & 4.989217 & -3.084971\end{array}$




$\begin{array}{crrr}\mathrm{C} & -1.115507 & 6.176886 & -1.686579 \\ \mathrm{Pd} & -3.706585 & 1.042216 & 1.139821 \\ \mathrm{P} & -4.986838 & -0.927586 & 1.111775 \\ \mathrm{C} & -6.292154 & -1.031424 & -0.161212 \\ \mathrm{O} & -3.951156 & 3.788831 & 2.474771 \\ \mathrm{C} & -3.235240 & 4.013051 & 3.478357 \\ \mathrm{O} & -2.115717 & 3.516980 & 3.733880 \\ \mathrm{C} & 0.048888 & -1.094739 & 1.602553 \\ \mathrm{C} & -3.988149 & -2.429783 & 0.819453 \\ \mathrm{C} & -5.871124 & -1.311436 & 2.663997 \\ \mathrm{H} & -3.242188 & 2.836966 & 1.559586 \\ \mathrm{H} & -0.129899 & 6.211961 & 1.386791 \\ \mathrm{H} & -0.327711 & 5.330286 & 2.937967 \\ \mathrm{H} & -1.753925 & 5.633982 & 1.895208 \\ \mathrm{H} & -5.566590 & 3.652838 & 0.633627 \\ \mathrm{H} & -6.657211 & 2.558602 & -0.294337 \\ \mathrm{H} & -5.900102 & 3.949780 & -1.113432 \\ \mathrm{H} & -2.842395 & 1.502967 & 5.222816 \\ \mathrm{H} & -3.900688 & 0.064185 & 5.076149 \\ \mathrm{H} & -4.306533 & 1.573600 & 4.187406 \\ \mathrm{H} & 0.864751 & -1.643674 & 1.103999 \\ \mathrm{H} & 0.014529 & -1.372434 & 2.666209 \\ \mathrm{H} & -0.908474 & -1.375704 & 1.134519 \\ \mathrm{H} & 2.727003 & 0.275501 & 1.524099 \\ \mathrm{H} & 2.315430 & 2.005414 & 1.813478 \\ \mathrm{H} & 2.054090 & 0.804587 & 3.106163 \\ \mathrm{H} & 1.187300 & 0.165974 & -0.805311 \\ \mathrm{H} & -0.530619 & 0.711302 & -0.858784 \\ \mathrm{H} & 0.781098 & 1.908428 & -0.644832 \\ \mathrm{H} & -6.510864 & -2.200540 & 2.536919 \\ \mathrm{H} & -5.140006 & -1.511879 & 3.462319 \\ \mathrm{H} & -6.496152 & -0.457148 & 2.966811 \\ \mathrm{H} & -3.229023 & -2.521907 & 1.612518 \\ \mathrm{H} & -4.623628 & -3.331179 & 0.819918 \\ \mathrm{H} & -3.475650 & -2.353101 & -0.152327 \\ \mathrm{H} & -7.032893 & -0.232846 & -0.000799 \\ \mathrm{H} & -5.846527 & -0.892236 & -1.158196 \\ \mathrm{H} & -6.804004 & -2.007259 & -0.119834 \\ \mathrm{H} & -3.638077 & 4.736176 & 4.229173 \\ \mathrm{H} & -0.948996 & 6.714736 & -2.634873 \\ \mathrm{H} & -1.745782 & 6.794881 & -1.028340 \\ \mathrm{H} & -0.144886 & 6.014209 & -1.192285 \\ \mathrm{H} & -2.975408 & 5.559742 & -3.963723 \\ \mathrm{H} & -3.818782 & 4.067071 & -3.420365 \\ \mathrm{H} & -4.048815 & 5.597149 & -2.527860 \\ \mathrm{H} & -0.513969 & 4.348048 & -3.989883 \\ \mathrm{H} & 0.198921 & 3.524085 & -2.564176 \\ \mathrm{H} & -1.159207 & 2.762742 & -3.444292\end{array}$

IV

62

scf done: -3269.420084
$\begin{array}{llll}\text { C } & -0.015779 & 0.004587 & -0.017597 \\ \text { P } & 0.017115 & -0.014937 & 1.805096\end{array}$
$\begin{array}{llll}\mathrm{P} & 0.017115 & -0.014937 & 1.805096\end{array}$
$\begin{array}{llll}\text { C } & 1.804406 & -0.070435 & 2.171271\end{array}$
$\begin{array}{llll}\text { C } & -0.488852 & 1.666987 & 2.286935\end{array}$
$\begin{array}{llll}\text { Pd } & -1.257100 & -1.605687 & 2.876925\end{array}$
$\begin{array}{llll}\mathrm{O} & -2.792852 & -2.581709 & 3.941792\end{array}$
$\begin{array}{llll}\text { C } & -2.736971 & -3.776245 & 4.385025\end{array}$ 


$\begin{array}{cccc}\mathrm{O} & -1.818515 & -4.584750 & 4.285290 \\ \mathrm{~S} & 0.010511 & -1.169200 & 4.853768 \\ \mathrm{Pd} & 1.081282 & -3.225790 & 4.008643 \\ \mathrm{P} & 2.790703 & -3.116988 & 5.584256 \\ \mathrm{C} & 2.185753 & -3.193226 & 7.303856 \\ \mathrm{~S} & -1.917241 & -2.570388 & 0.798344 \\ \mathrm{Pd} & -0.069960 & -4.042924 & 1.482679 \\ \mathrm{P} & 0.006042 & -5.092270 & -0.595420 \\ \mathrm{C} & -1.574403 & -5.851577 & -1.100391 \\ \mathrm{C} & -1.040894 & -1.715739 & 6.243177 \\ \mathrm{C} & -3.400061 & -3.568179 & 1.174874 \\ \mathrm{~S} & 1.712694 & -5.059860 & 2.655996 \\ \mathrm{C} & 0.833568 & -6.434107 & 3.492158 \\ \mathrm{C} & 0.398436 & -3.962274 & -1.973711 \\ \mathrm{C} & 1.220196 & -6.442422 & -0.772666 \\ \mathrm{C} & 4.054175 & -4.430648 & 5.508121 \\ \mathrm{C} & 3.762596 & -1.573679 & 5.529970 \\ \mathrm{H} & -0.374577 & -3.407559 & 3.075065 \\ \mathrm{H} & -3.844221 & -3.885492 & 0.219502 \\ \mathrm{H} & -4.118270 & -2.945913 & 1.725244 \\ \mathrm{H} & -3.144330 & -4.448363 & 1.781906 \\ \mathrm{H} & -0.147574 & -6.100425 & 3.862071 \\ \mathrm{H} & 1.457911 & -6.787835 & 4.326337 \\ \mathrm{H} & 0.714772 & -7.258655 & 2.773039 \\ \mathrm{H} & -2.069290 & -1.369259 & 6.074200 \\ \mathrm{H} & -0.643753 & -1.265891 & 7.165447 \\ \mathrm{H} & -1.040062 & -2.812437 & 6.322658 \\ \mathrm{H} & 2.327112 & 0.735654 & 1.630472 \\ \mathrm{H} & 1.968677 & 0.054600 & 3.252948 \\ \mathrm{H} & 2.212829 & -1.047627 & 1.864577 \\ \mathrm{H} & 0.121771 & 2.422730 & 1.765514 \\ \mathrm{H} & -1.550257 & 1.818975 & 2.039334 \\ \mathrm{H} & -0.368872 & 1.780707 & 3.375110 \\ \mathrm{H} & 0.605079 & 0.837128 & -0.387312 \\ \mathrm{H} & 0.388638 & -0.943830 & -0.406515 \\ \mathrm{H} & -1.045446 & 0.123439 & -0.386130 \\ \mathrm{H} & 3.030849 & -3.195858 & 8.011984 \\ \mathrm{H} & 1.547432 & -2.320942 & 7.511239 \\ \mathrm{H} & 1.587026 & -4.105252 & 7.452624 \\ \mathrm{H} & 3.094526 & -0.714914 & 5.699884 \\ \mathrm{H} & 4.546184 & -1.581850 & 6.305554 \\ \mathrm{H} & 4.235648 & -1.464179 & 4.541759 \\ \mathrm{H} & 3.577306 & -5.415148 & 5.632951 \\ \mathrm{H} & 4.554861 & -4.411585 & 4.528173 \\ \mathrm{H} & 4.805049 & -4.292564 & 6.303523 \\ \mathrm{H} & -3.672925 & -4.074502 & 4.929216 \\ \mathrm{H} & -1.459730 & -6.378521 & -2.062176 \\ \mathrm{H} & -1.914795 & -6.566251 & -0.335061 \\ \mathrm{H} & -2.340187 & -5.068310 & -1.210604 \\ \mathrm{H} & 1.191677 & -6.861518 & -1.792048 \\ \mathrm{H} & 2.231252 & -6.062807 & -0.559900 \\ \mathrm{H} & 0.995458 & -7.242200 & -0.050175 \\ \mathrm{H} & 0.411879 & -4.509497 & -2.930918 \\ \mathrm{H} & -0.363389 & -3.168165 & -2.020158 \\ \mathrm{H} & 1.383565 & -3.498490 & -1.810370\end{array}$

V

62

scf done: -3269.423182 


\begin{tabular}{|c|c|c|c|}
\hline$P$ & -5.127574 & 1.713437 & 1.192159 \\
\hline $\mathrm{Pd}$ & -3.987967 & 3.167986 & -0.178119 \\
\hline S & -3.438788 & 4.163129 & -2.362482 \\
\hline $\mathrm{C}$ & -4.987901 & 4.934896 & -2.931717 \\
\hline S & -2.329120 & 3.819905 & 1.663044 \\
\hline $\mathrm{Pd}$ & -1.363859 & 5.848225 & 0.892415 \\
\hline $\mathrm{O}$ & -3.327559 & 6.747531 & 0.928220 \\
\hline $\mathrm{C}$ & -3.978221 & 7.203921 & -0.035175 \\
\hline $\mathrm{O}$ & -3.691942 & 7.134569 & -1.253697 \\
\hline $\mathrm{Pd}$ & -2.018978 & 5.988834 & -1.891380 \\
\hline $\mathrm{P}$ & -0.392693 & 4.782164 & -2.915439 \\
\hline $\mathrm{C}$ & -0.312074 & 3.027103 & -2.419818 \\
\hline $\mathrm{C}$ & -3.406496 & 4.460025 & 2.991028 \\
\hline S & -0.478089 & 7.453742 & -0.700109 \\
\hline $\mathrm{C}$ & -1.346169 & 9.039792 & -0.448132 \\
\hline $\mathrm{P}$ & 0.642825 & 5.106512 & 1.624021 \\
\hline $\mathrm{C}$ & 1.258177 & 3.517604 & 0.974797 \\
\hline $\mathrm{C}$ & 1.319183 & 5.382383 & -2.720256 \\
\hline $\mathrm{C}$ & -0.629196 & 4.727969 & -4.719545 \\
\hline $\mathrm{C}$ & 2.036820 & 6.258455 & 1.395046 \\
\hline $\mathrm{C}$ & 0.592098 & 4.845782 & 3.427426 \\
\hline $\mathrm{H}$ & -5.129023 & 2.645105 & -1.111230 \\
\hline $\mathrm{H}$ & -5.694396 & 4.126360 & -3.169375 \\
\hline $\mathrm{H}$ & -4.781041 & 5.519656 & -3.838985 \\
\hline $\mathrm{H}$ & -5.411496 & 5.596859 & -2.167079 \\
\hline $\mathrm{H}$ & -4.141271 & 5.170485 & 2.591153 \\
\hline $\mathrm{H}$ & -2.786993 & 4.976653 & 3.739735 \\
\hline $\mathrm{H}$ & -3.911330 & 3.618332 & 3.489156 \\
\hline $\mathrm{H}$ & -2.096305 & 9.184392 & -1.237328 \\
\hline $\mathrm{H}$ & -0.600020 & 9.844818 & -0.493419 \\
\hline $\mathrm{H}$ & -1.842530 & 9.056421 & 0.531146 \\
\hline $\mathrm{H}$ & 2.009027 & 4.741941 & -3.293482 \\
\hline $\mathrm{H}$ & 1.396369 & 6.418746 & -3.081229 \\
\hline $\mathrm{H}$ & 1.612960 & 5.372908 & -1.659291 \\
\hline $\mathrm{H}$ & 0.169868 & 4.137486 & -5.196746 \\
\hline $\mathrm{H}$ & -1.605792 & 4.270344 & -4.936868 \\
\hline $\mathrm{H}$ & -0.619792 & 5.749334 & -5.128075 \\
\hline $\mathrm{H}$ & 0.607175 & 2.559404 & -2.807953 \\
\hline $\mathrm{H}$ & -0.345205 & 2.935358 & -1.322880 \\
\hline $\mathrm{H}$ & -1.187074 & 2.496901 & -2.825545 \\
\hline $\mathrm{H}$ & 2.954502 & 5.832650 & 1.832487 \\
\hline $\mathrm{H}$ & 2.194830 & 6.469517 & 0.327183 \\
\hline $\mathrm{H}$ & 1.805733 & 7.213286 & 1.890900 \\
\hline $\mathrm{H}$ & 1.495176 & 3.604488 & -0.096999 \\
\hline $\mathrm{H}$ & 2.171637 & 3.219613 & 1.515136 \\
\hline $\mathrm{H}$ & 0.488079 & 2.742820 & 1.111965 \\
\hline $\mathrm{H}$ & 0.261051 & 5.767474 & 3.929589 \\
\hline $\mathrm{H}$ & -0.121258 & 4.039247 & 3.655144 \\
\hline $\mathrm{H}$ & 1.591700 & 4.569935 & 3.801712 \\
\hline $\mathrm{H}$ & -4.917412 & 7.747540 & 0.217115 \\
\hline $\mathrm{C}$ & -4.143842 & 0.858991 & 2.474788 \\
\hline $\mathrm{C}$ & -6.528233 & 2.465686 & 2.092252 \\
\hline $\mathrm{C}$ & -5.916703 & 0.311331 & 0.330819 \\
\hline $\mathrm{H}$ & -7.033145 & 1.714215 & 2.720990 \\
\hline $\mathrm{H}$ & -6.188916 & 3.299185 & 2.724227 \\
\hline $\mathrm{H}$ & -7.247695 & 2.866527 & 1.362382 \\
\hline $\mathrm{H}$ & -4.794443 & 0.206902 & 3.080345 \\
\hline $\mathrm{H}$ & -3.379517 & 0.240498 & 1.979701 \\
\hline $\mathrm{H}$ & -3.627824 & 1.573228 & 3.129055 \\
\hline $\mathrm{H}$ & -6.388059 & -0.373649 & 1.054342 \\
\hline
\end{tabular}




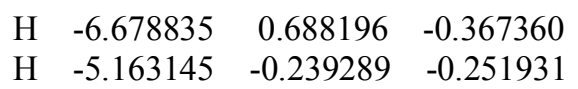




$\begin{array}{rrrr}\mathrm{C} & 1.720949 & 5.662447 & -0.168963 \\ \mathrm{C} & 2.012867 & 4.352575 & -0.823240 \\ \mathrm{C} & 1.063092 & 3.431596 & -1.053667 \\ \mathrm{C} & 1.177224 & 2.221063 & -1.869341 \\ \mathrm{C} & 0.005027 & 1.537138 & -2.231816 \\ \mathrm{H} & 5.308144 & 3.550715 & 3.410825 \\ \mathrm{C} & 0.041656 & 0.428202 & -3.073006 \\ \mathrm{C} & 1.260340 & -0.028199 & -3.569158 \\ \mathrm{C} & 2.436767 & 0.631391 & -3.206070 \\ \mathrm{C} & 2.402982 & 1.734759 & -2.359650 \\ \mathrm{H} & 1.603740 & 6.428375 & -0.958361 \\ \mathrm{H} & 0.752066 & 5.615027 & 0.370216 \\ \mathrm{H} & 1.656223 & 5.488090 & 2.314601 \\ \mathrm{H} & 2.900931 & 6.721996 & 2.616639 \\ \mathrm{H} & 3.021881 & 4.243934 & -1.244925 \\ \mathrm{H} & 0.060148 & 3.617659 & -0.638427 \\ \mathrm{H} & -0.959168 & 1.913298 & -1.870345 \\ \mathrm{H} & -0.889277 & -0.070738 & -3.357852 \\ \mathrm{H} & 1.294744 & -0.884353 & -4.248943 \\ \mathrm{H} & 3.393856 & 0.288757 & -3.614275 \\ \mathrm{H} & 3.337957 & 2.242956 & -2.094627\end{array}$

$\begin{array}{cccc}\text { VII } \\ \text { 74 } \\ \text { scf done: }-3347.445787 \\ \text { C } & 0.372892 & 0.116357 & -0.149138 \\ \text { C } & 0.159222 & 0.018835 & 1.221494 \\ \text { C } & 1.243869 & -0.007611 & 2.113887 \\ \text { C } & 2.541841 & 0.077077 & 1.587677 \\ \text { C } & 2.754955 & 0.175044 & 0.214825 \\ \text { C } & 1.670403 & 0.195308 & -0.659409 \\ \text { C } & 1.079611 & -0.120514 & 3.569427 \\ \text { Pd } & 0.176434 & 2.002520 & 4.189207 \\ \text { S } & -2.238123 & 2.331258 & 3.938149 \\ \text { C } & -2.912249 & 2.498348 & 5.619216 \\ \text { C } & -0.073143 & -0.371794 & 4.256296 \\ \text { C } & -0.070778 & -0.700686 & 5.711806 \\ \text { O } & -1.254294 & -0.230760 & 6.300912 \\ \text { C } & -1.185030 & -0.101980 & 7.696508 \\ \text { C } & -0.409418 & 1.063942 & 8.120102 \\ \text { C } & 0.219578 & 2.038329 & 8.469730 \\ \text { S } & 2.488305 & 2.813784 & 4.304378 \\ \text { Pd } & 1.455247 & 4.858036 & 3.576136 \\ \text { O } & 0.909121 & 5.449624 & 5.592562 \\ \text { C } & -0.258148 & 5.482892 & 6.048422 \\ \text { O } & -1.324872 & 5.207325 & 5.460573 \\ \text { Pd } & -1.572750 & 4.554895 & 3.365472 \\ \text { P } & -2.690149 & 4.257814 & 1.408925 \\ \text { C } & -2.493121 & 5.557149 & 0.146267 \\ \text { C } & 2.798255 & 3.008372 & 6.089763 \\ \text { S } & -0.165580 & 6.368039 & 2.594544 \\ \text { C } & -0.352681 & 7.775735 & 3.740069 \\ \text { P } & 2.416688 & 4.609914 & 1.522069 \\ \text { C } & 3.974681 & 3.675208 & 1.434884 \\ \text { C } & 2.821981 & 6.197528 & 0.726465 \\ \text { C } & 1.329217 & 3.755457 & 0.337749 \\ \text { C } & -2.383196 & 2.705162 & 0.505835 \\ \text { C } & -4.483783 & 4.236261 & 1.728566\end{array}$




$\begin{array}{rrrr}\text { H } & -0.700671 & 8.638704 & 3.154944 \\ \mathrm{H} & 0.618751 & 8.009593 & 4.196971 \\ \mathrm{H} & -1.084372 & 7.541599 & 4.523786 \\ \mathrm{H} & -2.289358 & 3.168098 & 6.226213 \\ \mathrm{H} & -2.932343 & 1.489440 & 6.051934 \\ \mathrm{H} & -3.933151 & 2.900652 & 5.548029 \\ \mathrm{H} & 2.173561 & 3.814901 & 6.496512 \\ \mathrm{H} & 3.861115 & 3.251634 & 6.230707 \\ \mathrm{H} & 2.565514 & 2.058602 & 6.596342 \\ \mathrm{H} & 4.334231 & 3.665865 & 0.392173 \\ \mathrm{H} & 4.729758 & 4.144621 & 2.083318 \\ \mathrm{H} & 3.814383 & 2.642329 & 1.778938 \\ \mathrm{H} & 3.281803 & 6.019566 & -0.259889 \\ \mathrm{H} & 1.908647 & 6.799664 & 0.610119 \\ \mathrm{H} & 3.528622 & 6.756638 & 1.358591 \\ \mathrm{H} & 1.830256 & 3.645921 & -0.638940 \\ \mathrm{H} & 1.081080 & 2.753364 & 0.727693 \\ \mathrm{H} & 0.400263 & 4.333139 & 0.208407 \\ \mathrm{H} & -0.350940 & 5.804284 & 7.113993 \\ \mathrm{H} & -3.138012 & 5.331486 & -0.719164 \\ \mathrm{H} & -2.778945 & 6.531111 & 0.571338 \\ \mathrm{H} & -1.447404 & 5.628984 & -0.188186 \\ \mathrm{H} & -5.041577 & 4.152607 & 0.780868 \\ \mathrm{H} & -4.723931 & 3.378696 & 2.375187 \\ \mathrm{H} & -4.782353 & 5.159733 & 2.247498 \\ \mathrm{H} & -3.020593 & 2.662833 & -0.392969 \\ \mathrm{H} & -1.325376 & 2.638428 & 0.208751 \\ \mathrm{H} & -2.618246 & 1.850396 & 1.159709 \\ \mathrm{H} & 0.771323 & 2.895681 & 8.810985 \\ \mathrm{H} & 0.007776 & -1.801866 & 5.853211 \\ \mathrm{H} & 0.824029 & -0.249117 & 6.192188 \\ \mathrm{H} & -0.769295 & -1.019602 & 8.162302 \\ \mathrm{H} & -2.221086 & -0.008830 & 8.064570 \\ \mathrm{H} & -1.021986 & -0.549489 & 3.733136 \\ \mathrm{H} & 2.019879 & -0.127755 & 4.138882 \\ \mathrm{H} & 3.396410 & 0.045416 & 2.273216 \\ \mathrm{H} & 3.776157 & 0.222511 & -0.175096 \\ \mathrm{H} & 1.832670 & 0.257381 & -1.739047 \\ \mathrm{H} & -0.482757 & 0.116235 & -0.831555 \\ \mathrm{H} & -0.864327 & -0.049386 & 1.606413 \\ \mathrm{H} & 0.001707 & 3.531394 & 4.381466\end{array}$

\section{TS (VII-VIII)}

74

scf done: -3347.430650

$\begin{array}{crrr}\text { C } & -0.035571 & 0.030071 & 0.062640 \\ \text { C } & -0.023351 & 0.032915 & 1.455290 \\ \text { C } & 1.197303 & -0.007344 & 2.131791 \\ \text { C } & 2.392965 & -0.036167 & 1.424175 \\ \text { C } & 2.398388 & -0.023517 & 0.019782 \\ \text { C } & 1.163624 & 0.001802 & -0.644633 \\ C & 3.633511 & -0.037096 & -0.769153 \\ C & 4.856480 & -0.379062 & -0.340025 \\ C & 6.066585 & -0.421067 & -1.210607 \\ \text { O } & 7.189794 & 0.227488 & -0.653249 \\ \text { C } & 7.134466 & 1.615396 & -0.642641 \\ \text { C } & 6.377470 & 2.150278 & 0.521591 \\ \text { Pd } & 4.482196 & 3.195314 & 0.599109\end{array}$




\begin{tabular}{|c|c|c|c|}
\hline $\mathrm{S}$ & 2.105337 & 3.578684 & 1.041426 \\
\hline $\mathrm{C}$ & 1.909012 & 3.886433 & 2.825426 \\
\hline $\mathrm{C}$ & 6.081039 & 2.257030 & 1.734997 \\
\hline $\mathrm{S}$ & 5.170476 & 4.287003 & -1.599211 \\
\hline $\mathrm{C}$ & 6.742079 & 5.053195 & -1.059568 \\
\hline $\mathrm{Pd}$ & 3.805211 & 6.233036 & -1.655887 \\
\hline $\mathrm{O}$ & 4.568567 & 6.782581 & 0.297080 \\
\hline $\mathrm{C}$ & 3.950136 & 6.914992 & 1.373522 \\
\hline $\mathrm{O}$ & 2.752142 & 6.632726 & 1.603495 \\
\hline $\mathrm{Pd}$ & 1.613742 & 5.682634 & 0.083775 \\
\hline $\mathrm{P}$ & 0.207725 & 4.542154 & -1.281970 \\
\hline $\mathrm{C}$ & -0.596232 & 5.518346 & -2.593959 \\
\hline $\mathrm{S}$ & 1.825556 & 7.602526 & -1.412549 \\
\hline $\mathrm{C}$ & 2.266686 & 9.047941 & -0.387296 \\
\hline $\mathrm{P}$ & 3.788470 & 6.141442 & -3.919831 \\
\hline $\mathrm{C}$ & 2.874355 & 7.475661 & -4.759457 \\
\hline $\mathrm{C}$ & 1.016360 & 3.167927 & -2.159426 \\
\hline $\mathrm{C}$ & -1.176240 & 3.762572 & -0.393706 \\
\hline $\mathrm{C}$ & 5.498889 & 6.361279 & -4.510881 \\
\hline $\mathrm{C}$ & 3.245403 & 4.624435 & -4.773460 \\
\hline $\mathrm{H}$ & 4.319923 & 2.588703 & 2.029011 \\
\hline $\mathrm{H}$ & 2.196845 & 2.969692 & 3.362810 \\
\hline $\mathrm{H}$ & 0.850571 & 4.106810 & 3.027626 \\
\hline $\mathrm{H}$ & 2.528228 & 4.732810 & 3.149497 \\
\hline $\mathrm{H}$ & 6.601142 & 5.582999 & -0.107553 \\
\hline $\mathrm{H}$ & 7.076520 & 5.769325 & -1.825091 \\
\hline $\mathrm{H}$ & 7.513107 & 4.276983 & -0.949705 \\
\hline $\mathrm{H}$ & 2.114520 & 8.825936 & 0.677586 \\
\hline $\mathrm{H}$ & 1.617126 & 9.882034 & -0.687694 \\
\hline $\mathrm{H}$ & 3.316906 & 9.322046 & -0.558617 \\
\hline $\mathrm{H}$ & -1.246720 & 4.870063 & -3.204092 \\
\hline $\mathrm{H}$ & -1.199157 & 6.321350 & -2.143244 \\
\hline $\mathrm{H}$ & 0.162595 & 5.990704 & -3.236242 \\
\hline $\mathrm{H}$ & -1.829716 & 3.232957 & -1.107147 \\
\hline $\mathrm{H}$ & -0.774083 & 3.043274 & 0.337141 \\
\hline $\mathrm{H}$ & -1.762648 & 4.527546 & 0.137499 \\
\hline $\mathrm{H}$ & 1.886022 & 3.545108 & -2.717541 \\
\hline $\mathrm{H}$ & 1.368921 & 2.424293 & -1.425960 \\
\hline $\mathrm{H}$ & 0.307567 & 2.681874 & -2.851356 \\
\hline $\mathrm{H}$ & 3.018950 & 7.397380 & -5.849781 \\
\hline $\mathrm{H}$ & 1.800591 & 7.427500 & -4.526035 \\
\hline $\mathrm{H}$ & 3.249843 & 8.450514 & -4.412412 \\
\hline $\mathrm{H}$ & 2.155983 & 4.496355 & -4.681989 \\
\hline $\mathrm{H}$ & 3.500646 & 4.694374 & -5.843925 \\
\hline $\mathrm{H}$ & 3.754292 & 3.750777 & -4.337063 \\
\hline $\mathrm{H}$ & 5.935459 & 7.271948 & -4.072499 \\
\hline $\mathrm{H}$ & 6.101557 & 5.492887 & -4.203938 \\
\hline $\mathrm{H}$ & 5.510816 & 6.445770 & -5.610219 \\
\hline $\mathrm{H}$ & 4.525693 & 7.340328 & 2.228063 \\
\hline $\mathrm{H}$ & 6.233706 & 2.155028 & 2.801519 \\
\hline $\mathrm{H}$ & 6.716513 & 2.013546 & -1.590871 \\
\hline $\mathrm{H}$ & 8.174223 & 1.981177 & -0.560744 \\
\hline $\mathrm{H}$ & 6.388864 & -1.466393 & -1.359282 \\
\hline $\mathrm{H}$ & 5.834034 & -0.002365 & -2.212911 \\
\hline $\mathrm{H}$ & 5.016196 & -0.696347 & 0.699603 \\
\hline $\mathrm{H}$ & 3.518635 & 0.242591 & -1.828781 \\
\hline $\mathrm{H}$ & 1.151969 & -0.018247 & -1.740579 \\
\hline $\mathrm{H}$ & -0.987494 & 0.034285 & -0.478070 \\
\hline $\mathrm{H}$ & -0.962118 & 0.047042 & 2.016213 \\
\hline $\mathrm{H}$ & 1.213987 & -0.022187 & 3.225496 \\
\hline
\end{tabular}


H $\quad 3.341762 \quad-0.065255 \quad 1.970576$

$\begin{array}{cccc}\text { VIII } & & \\ \text { 74 } & & & \\ \text { scf done: }-3347.484708 & \\ \mathrm{C} & -1.758674 & -0.495212 & 0.981993 \\ \mathrm{C} & -0.931270 & -0.062191 & 2.010196 \\ \mathrm{C} & 0.419457 & 0.232935 & 1.762410 \\ \mathrm{C} & 0.912871 & 0.082938 & 0.459213 \\ \mathrm{C} & 0.084307 & -0.356338 & -0.569696 \\ \mathrm{C} & -1.253621 & -0.643312 & -0.311418 \\ \mathrm{C} & 1.328777 & 0.686798 & 2.822740 \\ \mathrm{C} & 1.106722 & 0.518827 & 4.171055 \\ \mathrm{C} & 2.166842 & 0.543494 & 5.232389 \\ \mathrm{O} & 1.639994 & 0.803746 & 6.495903 \\ \mathrm{C} & 0.985836 & 2.040433 & 6.625080 \\ \mathrm{C} & -0.055283 & 2.264450 & 5.571808 \\ \mathrm{Pd} & 0.684775 & 2.753744 & 3.716517 \\ \mathrm{~S} & 1.299024 & 3.340328 & 1.259603 \\ \mathrm{C} & -0.423809 & 3.486952 & 0.694085 \\ \mathrm{C} & -1.367375 & 2.139590 & 5.761342 \\ \mathrm{~S} & 0.317231 & 4.926517 & 4.807093 \\ \mathrm{C} & -1.448501 & 5.314473 & 4.621470 \\ \mathrm{Pd} & 1.451492 & 6.653296 & 3.633099 \\ \mathrm{P} & 3.070475 & 6.546693 & 5.222588 \\ \mathrm{C} & 3.931151 & 4.943504 & 5.350343 \\ \mathrm{O} & -0.310053 & 7.102695 & 2.525279 \\ \mathrm{C} & -0.473569 & 6.881077 & 1.306385 \\ \mathrm{O} & 0.312983 & 6.305111 & 0.524526 \\ \mathrm{Pd} & 2.186329 & 5.525509 & 1.137682 \\ \mathrm{~S} & 2.906146 & 7.701250 & 1.970475 \\ \mathrm{C} & 1.939708 & 8.988557 & 1.109091 \\ \mathrm{P} & 4.273168 & 4.626360 & 1.121270 \\ \mathrm{C} & 4.718064 & 4.337387 & -0.621499 \\ \mathrm{C} & 5.647218 & 5.641411 & 1.760787 \\ \mathrm{C} & 4.526454 & 2.993970 & 1.896596 \\ \mathrm{C} & 4.407748 & 7.774726 & 5.079620 \\ \mathrm{C} & 2.404897 & 6.828160 & 6.893345 \\ \mathrm{H} & -2.102569 & 2.250771 & 4.954254 \\ \mathrm{H} & -0.849754 & 2.470781 & 0.692144 \\ \mathrm{H} & -0.455864 & 3.899906 & -0.323197 \\ \mathrm{H} & -1.010288 & 4.129363 & 1.368222 \\ \mathrm{H} & -1.794539 & 5.186908 & 3.587070 \\ \mathrm{H} & -1.608228 & 6.357777 & 4.926680 \\ \mathrm{H} & -2.002564 & 4.644804 & 5.294677 \\ \mathrm{H} & 1.554207 & 8.601197 & 0.155920 \\ \mathrm{H} & 2.608971 & 9.839092 & 0.918536 \\ \mathrm{H} & 1.099635 & 9.315577 & 1.737019 \\ \mathrm{H} & 6.607594 & 5.138383 & 1.560746 \\ \mathrm{H} & 5.636344 & 6.627513 & 1.272602 \\ \mathrm{H} & 5.546822 & 5.797936 & 2.846201 \\ \mathrm{H} & 5.730486 & 3.906794 & -0.698612 \\ \mathrm{H} & 3.987117 & 3.645253 & -1.067689 \\ \mathrm{H} & 4.681239 & 5.286248 & -1.177952 \\ \mathrm{H} & 5.585330 & 2.698994 & 1.815093 \\ \mathrm{H} & 4.226776 & 3.005998 & 2.956674 \\ \mathrm{H} & 3.903530 & 2.254804 & 1.368777 \\ \mathrm{H} & 5.132238 & 7.641838 & 5.899847\end{array}$




$\begin{array}{rrrr}\mathrm{H} & 4.917004 & 7.689236 & 4.108274 \\ \mathrm{H} & 3.974210 & 8.785132 & 5.137369 \\ \mathrm{H} & 4.524851 & 4.765189 & 4.440434 \\ \mathrm{H} & 4.603709 & 4.933600 & 6.223977 \\ \mathrm{H} & 3.191915 & 4.131816 & 5.450530 \\ \mathrm{H} & 1.892029 & 7.801445 & 6.928190 \\ \mathrm{H} & 1.674687 & 6.040994 & 7.134534 \\ \mathrm{H} & 3.222985 & 6.820626 & 7.632327 \\ \mathrm{H} & -1.429980 & 7.243179 & 0.863648 \\ \mathrm{H} & -1.767345 & 1.913289 & 6.760719 \\ \mathrm{H} & 1.724980 & 2.872775 & 6.615716 \\ \mathrm{H} & 0.514979 & 2.035590 & 7.623866 \\ \mathrm{H} & 2.625735 & -0.459720 & 5.298779 \\ \mathrm{H} & 2.981057 & 1.253267 & 4.957384 \\ \mathrm{H} & 0.185966 & 0.014737 & 4.486914 \\ \mathrm{H} & 2.349024 & 0.933706 & 2.497030 \\ \mathrm{H} & 1.964500 & 0.311755 & 0.256973 \\ \mathrm{H} & 0.486462 & -0.476039 & -1.579306 \\ \mathrm{H} & -1.907012 & -0.986719 & -1.117915 \\ \mathrm{H} & -2.808455 & -0.720621 & 1.188327 \\ \mathrm{H} & -1.342503 & 0.056254 & 3.018792\end{array}$

\section{TS (VIII-IX)}

74

scf done: -3347.478572

$\begin{array}{llll}\text { C } & 1.225998 & -0.038642 & -0.800907\end{array}$

C $\quad 0.003042 \quad-0.072400 \quad-0.113686$

$\begin{array}{llll}\text { C } & -0.031660 & -0.092518 & 1.277290\end{array}$

$\begin{array}{llll}\text { C } & 1.154906 & -0.071227 & 2.007049\end{array}$

$\begin{array}{llll}\text { C } & 2.378912 & -0.037977 & 1.336528\end{array}$

$\begin{array}{llll}\text { C } & 2.414918 & -0.023813 & -0.052347\end{array}$

$\begin{array}{llll}\text { C } & 1.229049 & -0.008045 & -2.269185\end{array}$

C $\quad 2.325582 \quad-0.452452 \quad-3.052564$

C $\quad 2.106805 \quad-1.029120 \quad-4.427111$

$\begin{array}{llll}\text { O } & 3.257812 & -0.916341 & -5.203314\end{array}$

$\begin{array}{llll}\text { C } & 3.762089 & 0.393000 & -5.155033\end{array}$

$\begin{array}{llll}\text { C } & 3.722124 & 0.899748 & -3.745833\end{array}$

$\begin{array}{llll}\text { C } & 4.801065 & 1.150421 & -2.997114\end{array}$

$\begin{array}{llll}\mathrm{Pd} & 1.935767 & 1.855252 & -3.092375\end{array}$

$\begin{array}{llll}\mathrm{S} & -0.079586 & 2.800160 & -1.945929\end{array}$

$\begin{array}{llll}\text { C } & 0.744346 & 3.461412 & -0.464469\end{array}$

$\begin{array}{llll}\text { S } & 2.996939 & 3.733069 & -4.402633\end{array}$

C $4.085619 \quad 4.526503 \quad-3.181858$

$\begin{array}{llll}\mathrm{Pd} & 1.356699 & 5.355180 & -4.943957\end{array}$

$\begin{array}{llll}\text { O } & 1.935268 & 6.581147 & -3.315356\end{array}$

$\begin{array}{llll}\text { C } & 1.322971 & 6.621627 & -2.226774\end{array}$

$\begin{array}{llll}\text { O } & 0.294607 & 5.988378 & -1.905057\end{array}$

$\begin{array}{llll}\mathrm{Pd} & -0.792228 & 4.686522 & -3.187297\end{array}$

$\begin{array}{llll}\mathrm{P} & -2.446663 & 3.402461 & -4.069043\end{array}$

$\begin{array}{llll}\text { C } & -2.164706 & 1.605799 & -4.221869\end{array}$

$\begin{array}{llll}\mathrm{P} & 1.136421 & 4.333567 & -6.957154\end{array}$

$\begin{array}{llll}\text { C } & 2.715489 & 4.220951 & -7.855757\end{array}$

$\begin{array}{llll}\text { S } & -0.877001 & 6.345971 & -4.977258\end{array}$

C $\quad-0.747562 \quad 8.005153 \quad-4.225261$

$\begin{array}{llll}\text { C } & 0.525282 & 2.616496 & -6.888228\end{array}$

$\begin{array}{llll}\text { C } & 0.022011 & 5.157864 & -8.139382\end{array}$

$\begin{array}{llll}\text { C } & -3.872612 & 3.508757 & -2.940174\end{array}$

C $\quad-3.129847 \quad 3.883038 \quad-5.690250$ 


$\begin{array}{rrrr}\text { H } & 4.734080 & 1.432580 & -1.938641 \\ \mathrm{H} & 1.067068 & 2.597103 & 0.137092 \\ \mathrm{H} & 0.032119 & 4.065100 & 0.114411 \\ \mathrm{H} & 1.612925 & 4.080159 & -0.727972 \\ \mathrm{H} & 3.547573 & 4.785583 & -2.259300 \\ \mathrm{H} & 4.519828 & 5.437573 & -3.615918 \\ \mathrm{H} & 4.891097 & 3.812030 & -2.955014 \\ \mathrm{H} & -1.117817 & 7.979464 & -3.191085 \\ \mathrm{H} & -1.363494 & 8.693353 & -4.820796 \\ \mathrm{H} & 0.297809 & 8.342909 & -4.230056 \\ \mathrm{H} & -4.003953 & 3.254927 & -5.928287 \\ \mathrm{H} & -3.426270 & 4.942483 & -5.668226 \\ \mathrm{H} & -2.376214 & 3.760515 & -6.484028 \\ \mathrm{H} & -4.712122 & 2.900470 & -3.316171 \\ \mathrm{H} & -3.568494 & 3.144642 & -1.946495 \\ \mathrm{H} & -4.193731 & 4.557018 & -2.844042 \\ \mathrm{H} & -3.055724 & 1.123560 & -4.656123 \\ \mathrm{H} & -1.289095 & 1.397508 & -4.855816 \\ \mathrm{H} & -1.976359 & 1.186594 & -3.221136 \\ \mathrm{H} & 0.003655 & 4.602714 & -9.091772 \\ \mathrm{H} & -0.995580 & 5.231687 & -7.726454 \\ \mathrm{H} & 0.381642 & 6.182313 & -8.321505 \\ \mathrm{H} & -0.534166 & 2.625919 & -6.588848 \\ \mathrm{H} & 0.617107 & 2.125680 & -7.871221 \\ \mathrm{H} & 1.093249 & 2.046966 & -6.133896 \\ \mathrm{H} & 3.143574 & 5.228144 & -7.974179 \\ \mathrm{H} & 3.425473 & 3.611486 & -7.276589 \\ \mathrm{H} & 2.555413 & 3.771718 & -8.849786 \\ \mathrm{H} & 1.738342 & 7.309618 & -1.454589 \\ \mathrm{H} & 5.810154 & 1.087570 & -3.427371 \\ \mathrm{H} & 3.176764 & 1.066265 & -5.820189 \\ \mathrm{H} & 4.796985 & 0.365891 & -5.535516 \\ \mathrm{H} & 1.874739 & -2.104550 & -4.342485 \\ \mathrm{H} & 1.229784 & -0.534099 & -4.903916 \\ \mathrm{H} & 3.144883 & -0.930046 & -2.505241 \\ \mathrm{H} & 0.234258 & -0.067764 & -2.736757 \\ \mathrm{H} & -0.930074 & -0.088987 & -0.686686 \\ \mathrm{H} & -0.993195 & -0.128692 & 1.796651 \\ \mathrm{H} & 1.128269 & -0.087104 & 3.099671 \\ \mathrm{H} & 3.313148 & -0.027035 & 1.904450 \\ \mathrm{H} & 3.383791 & 0.003346 & -0.563933\end{array}$

IX

74

scf done: -3347.519137

$\begin{array}{cccc}\mathrm{C} & -0.048232 & -0.117447 & 0.331203 \\ \mathrm{C} & -0.047219 & -0.200605 & 1.723336 \\ \mathrm{C} & 1.147020 & -0.110714 & 2.429976 \\ \mathrm{C} & 2.371046 & 0.078461 & 1.764499 \\ \mathrm{C} & 2.351706 & 0.144607 & 0.359313 \\ \mathrm{C} & 1.158348 & 0.044007 & -0.347998 \\ \mathrm{C} & 3.649770 & 0.203282 & 2.483589 \\ \mathrm{Pd} & 3.861445 & 2.127106 & 3.390538 \\ \mathrm{~S} & 4.131155 & 4.144567 & 5.006013 \\ \mathrm{Pd} & 5.251832 & 5.885970 & 3.859438 \\ \mathrm{~S} & 6.572409 & 6.861782 & 2.052558 \\ \mathrm{C} & 5.744492 & 8.380149 & 1.467524 \\ \mathrm{C} & 3.894732 & -0.625586 & 3.726179\end{array}$




\begin{tabular}{|c|c|c|c|}
\hline $\mathrm{C}$ & 5.275751 & -1.298451 & 3.799641 \\
\hline $\mathrm{O}$ & 5.706903 & -1.143197 & 5.126682 \\
\hline $\mathrm{C}$ & 5.353235 & 0.153276 & 5.510577 \\
\hline $\mathrm{C}$ & 4.009542 & 0.376427 & 4.861678 \\
\hline $\mathrm{C}$ & 3.019712 & 1.214168 & 5.289858 \\
\hline S & 4.050865 & 2.970667 & 1.098019 \\
\hline $\mathrm{Pd}$ & 5.344767 & 4.961317 & 1.157449 \\
\hline $\mathrm{P}$ & 7.200492 & 3.686097 & 0.851892 \\
\hline $\mathrm{C}$ & 7.239521 & 2.052724 & 1.664364 \\
\hline $\mathrm{C}$ & 2.328218 & 3.540607 & 0.916098 \\
\hline $\mathrm{C}$ & 2.412783 & 4.722767 & 5.188485 \\
\hline $\mathrm{P}$ & 7.025380 & 5.512129 & 5.214433 \\
\hline $\mathrm{C}$ & 6.597747 & 5.892945 & 6.942067 \\
\hline $\mathrm{O}$ & 3.398226 & 6.638990 & 3.094186 \\
\hline $\mathrm{C}$ & 3.050718 & 6.688731 & 1.896359 \\
\hline $\mathrm{O}$ & 3.625093 & 6.167398 & 0.914580 \\
\hline $\mathrm{C}$ & 7.652461 & 3.800804 & 5.272676 \\
\hline $\mathrm{C}$ & 8.510907 & 6.518542 & 4.901591 \\
\hline $\mathrm{C}$ & 7.350396 & 3.291016 & -0.919420 \\
\hline $\mathrm{C}$ & 8.806893 & 4.428582 & 1.287126 \\
\hline $\mathrm{H}$ & 1.990824 & 1.135569 & 4.918435 \\
\hline $\mathrm{H}$ & 1.691409 & 2.642518 & 0.866780 \\
\hline $\mathrm{H}$ & 2.232412 & 4.118053 & -0.013454 \\
\hline $\mathrm{H}$ & 2.025485 & 4.166405 & 1.768447 \\
\hline $\mathrm{H}$ & 1.956132 & 4.925580 & 4.210577 \\
\hline $\mathrm{H}$ & 2.391773 & 5.642822 & 5.789296 \\
\hline $\mathrm{H}$ & 1.842648 & 3.940422 & 5.712258 \\
\hline $\mathrm{H}$ & 5.112990 & 8.156677 & 0.597122 \\
\hline $\mathrm{H}$ & 6.525204 & 9.100363 & 1.185641 \\
\hline $\mathrm{H}$ & 5.125144 & 8.807877 & 2.268184 \\
\hline $\mathrm{H}$ & 9.624866 & 3.745962 & 1.004230 \\
\hline $\mathrm{H}$ & 8.928026 & 5.390503 & 0.766874 \\
\hline $\mathrm{H}$ & 8.856759 & 4.622188 & 2.370154 \\
\hline $\mathrm{H}$ & 8.249434 & 2.681123 & -1.108096 \\
\hline $\mathrm{H}$ & 6.453902 & 2.736393 & -1.237286 \\
\hline $\mathrm{H}$ & 7.408931 & 4.220422 & -1.505847 \\
\hline $\mathrm{H}$ & 8.200469 & 1.551439 & 1.462257 \\
\hline $\mathrm{H}$ & 7.094885 & 2.157364 & 2.751955 \\
\hline $\mathrm{H}$ & 6.419642 & 1.432891 & 1.268135 \\
\hline $\mathrm{H}$ & 9.287844 & 6.280339 & 5.646762 \\
\hline $\mathrm{H}$ & 8.899148 & 6.340363 & 3.887587 \\
\hline $\mathrm{H}$ & 8.251915 & 7.585712 & 4.976265 \\
\hline $\mathrm{H}$ & 8.132742 & 3.543702 & 4.315322 \\
\hline $\mathrm{H}$ & 8.394763 & 3.692089 & 6.080462 \\
\hline $\mathrm{H}$ & 6.816084 & 3.106386 & 5.453162 \\
\hline $\mathrm{H}$ & 6.241241 & 6.931812 & 7.014114 \\
\hline $\mathrm{H}$ & 5.789524 & 5.222215 & 7.269381 \\
\hline $\mathrm{H}$ & 7.479977 & 5.764415 & 7.590902 \\
\hline $\mathrm{H}$ & 2.123680 & 7.264987 & 1.671378 \\
\hline $\mathrm{H}$ & 3.171752 & 1.815478 & 6.192623 \\
\hline $\mathrm{H}$ & 6.095272 & 0.901852 & 5.147491 \\
\hline $\mathrm{H}$ & 5.315321 & 0.221834 & 6.609590 \\
\hline $\mathrm{H}$ & 5.264291 & -2.369522 & 3.553823 \\
\hline $\mathrm{H}$ & 5.978220 & -0.790292 & 3.101149 \\
\hline $\mathrm{H}$ & 3.093681 & -1.362869 & 3.919140 \\
\hline $\mathrm{H}$ & 4.505560 & 0.179409 & 1.790314 \\
\hline $\mathrm{H}$ & 3.296173 & 0.268420 & -0.180328 \\
\hline $\mathrm{H}$ & 1.170034 & 0.087103 & -1.440587 \\
\hline $\mathrm{H}$ & -0.987238 & -0.192780 & -0.223538 \\
\hline $\mathrm{H}$ & -0.987287 & -0.343931 & 2.263288 \\
\hline
\end{tabular}


X

74

scf done: -3347.522211

$\begin{array}{llll}\text { C } & 1.442985 & -1.263375 & 1.819262\end{array}$

$\begin{array}{llll}\text { C } & 0.552829 & -0.718016 & 2.765753\end{array}$

$\begin{array}{llll}\text { C } & 1.061618 & -0.386569 & 4.034785\end{array}$

$\begin{array}{llll}\text { C } & 2.405052 & -0.584951 & 4.336516\end{array}$

$\begin{array}{llll}\text { C } & 3.268206 & -1.134305 & 3.389162\end{array}$

$\begin{array}{llll}\text { C } & 2.780059 & -1.477052 & 2.127525\end{array}$

$\begin{array}{llll}\text { C } & -0.852483 & -0.504333 & 2.374928\end{array}$

$\begin{array}{llll}\mathrm{Pd} & -1.662207 & -2.411782 & 2.385534\end{array}$

$\begin{array}{llll}\mathrm{S} & -1.192633 & -3.294289 & 4.605462\end{array}$

$\begin{array}{llll}\mathrm{Pd} & -2.376327 & -5.078561 & 3.557163\end{array}$

$\begin{array}{llll}\mathrm{P} & -4.206944 & -4.629404 & 4.828672\end{array}$

$\begin{array}{llll}\text { C } & -5.591501 & -3.910700 & 3.890818\end{array}$

$\begin{array}{llll}\text { C } & -1.711279 & 0.420925 & 3.208086\end{array}$

$\begin{array}{llll}\text { C } & -3.169449 & 0.432758 & 2.807026\end{array}$

$\begin{array}{llll}\text { C } & -3.325620 & 1.537664 & 1.791291\end{array}$

$\begin{array}{llll}\text { O } & -2.057908 & 2.134582 & 1.673707\end{array}$

$\begin{array}{llll}\text { C } & -1.356208 & 1.882223 & 2.862759\end{array}$

$\begin{array}{llll}\text { C } & -4.139387 & -0.317599 & 3.328291\end{array}$

$\begin{array}{llll}\mathrm{S} & -2.484604 & -2.207973 & 0.125610\end{array}$

$\begin{array}{llll}\mathrm{Pd} & -3.107685 & -4.446586 & 0.650447\end{array}$

$\begin{array}{llll}\text { S } & -3.776826 & -6.346930 & 1.998971\end{array}$

$\begin{array}{llll}\mathrm{C} & -2.772055 & -7.778036 & 1.465852\end{array}$

$\begin{array}{llll}\text { C } & -1.051480 & -2.366600 & -0.986732\end{array}$

$\begin{array}{llll}\text { C } & 0.561321 & -3.789429 & 4.549414\end{array}$

$\begin{array}{llll}\mathrm{O} & -0.594743 & -5.763521 & 2.649130\end{array}$

$\begin{array}{llll}\text { C } & -0.328359 & -5.597188 & 1.439162\end{array}$

$\begin{array}{llll}\mathrm{O} & -1.043603 & -5.074145 & 0.558725\end{array}$

$\begin{array}{llll}\mathrm{P} & -5.181196 & -3.908321 & -0.114381\end{array}$

$\begin{array}{llll}\text { C } & -5.054062 & -3.701721 & -1.920940\end{array}$

$\begin{array}{llll}\text { C } & -6.480331 & -5.171543 & 0.075023\end{array}$

$\begin{array}{llll}\text { C } & -5.962643 & -2.363346 & 0.452523\end{array}$

$\begin{array}{llll}\text { C } & -4.876067 & -6.166464 & 5.539006\end{array}$

$\begin{array}{llll}\text { C } & -4.023615 & -3.520932 & 6.258870\end{array}$

$\begin{array}{llll}\mathrm{H} & -3.912439 & -1.059934 & 4.102837\end{array}$

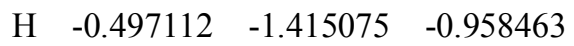

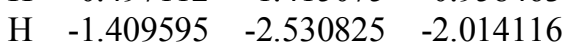

$\begin{array}{llll}\mathrm{H} & -0.405574 & -3.200738 & -0.677479\end{array}$

$\begin{array}{llll}\mathrm{H} & 0.946653 & -3.811461 & 3.521858\end{array}$

$\begin{array}{llll}\mathrm{H} & 0.680213 & -4.778609 & 5.012600\end{array}$

$\begin{array}{llll}\mathrm{H} & 1.117892 & -3.041431 & 5.133020\end{array}$

$\begin{array}{llll}\mathrm{H} & -2.481917 & -7.659526 & 0.412703\end{array}$

$\begin{array}{llll}\mathrm{H} & -3.386927 & -8.682237 & 1.575731\end{array}$

$\begin{array}{llll}\mathrm{H} & -1.870631 & -7.866759 & 2.086409\end{array}$

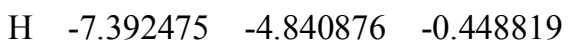

$\begin{array}{llll}\mathrm{H} & -6.135817 & -6.122335 & -0.358874\end{array}$

$\begin{array}{llll}\mathrm{H} & -6.716384 & -5.347702 & 1.134451\end{array}$

$\begin{array}{llll}\mathrm{H} & -6.053063 & -3.530634 & -2.356012\end{array}$

$\mathrm{H} \quad-4.403278 \quad-2.842723 \quad-2.144141$

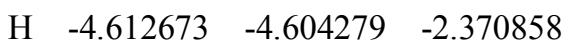

$\begin{array}{llll}\mathrm{H} & -6.859932 & -2.165309 & -0.156664\end{array}$

$\begin{array}{llll}\mathrm{H} & -6.257882 & -2.444510 & 1.508899\end{array}$

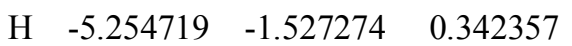

$\begin{array}{llll}\mathrm{H} & -5.773634 & -5.947254 & 6.140715\end{array}$ 


$\begin{array}{rrrr}\text { H } & -5.133317 & -6.862689 & 4.726579 \\ \mathrm{H} & -4.118526 & -6.640786 & 6.181346 \\ \mathrm{H} & -5.882425 & -4.612340 & 3.093502 \\ \mathrm{H} & -6.454545 & -3.727290 & 4.551492 \\ \mathrm{H} & -5.256968 & -2.963169 & 3.438443 \\ \mathrm{H} & -3.253757 & -3.912067 & 6.940326 \\ \mathrm{H} & -3.708026 & -2.517359 & 5.935128 \\ \mathrm{H} & -4.987747 & -3.449317 & 6.788779 \\ \mathrm{H} & 0.668698 & -5.968356 & 1.105687 \\ \mathrm{H} & -5.186920 & -0.197554 & 3.028551 \\ \mathrm{H} & -3.642045 & 1.169156 & 0.798251 \\ \mathrm{H} & -4.089673 & 2.268509 & 2.129856 \\ \mathrm{H} & -1.686018 & 2.553573 & 3.683863 \\ \mathrm{H} & -0.282521 & 2.050870 & 2.684790 \\ \mathrm{H} & -1.609405 & 0.196277 & 4.282970 \\ \mathrm{H} & -0.927552 & -0.236082 & 1.307050 \\ \mathrm{H} & 1.064040 & -1.513849 & 0.822147 \\ \mathrm{H} & 3.452942 & -1.897466 & 1.375157 \\ \mathrm{H} & 4.323916 & -1.285684 & 3.629658 \\ \mathrm{H} & 2.785277 & -0.301573 & 5.322148 \\ \mathrm{H} & 0.404592 & 0.045425 & 4.795697\end{array}$

\section{TS (X-XI)}

74

scf done: -3347.488424

$\begin{array}{cccc}\mathrm{C} & 0.021238 & 0.013755 & 0.002261 \\ \mathrm{C} & 0.020179 & -0.016607 & 1.407700 \\ \mathrm{C} & 1.257344 & 0.016032 & 2.071426 \\ \mathrm{C} & 2.450485 & 0.104866 & 1.362183 \\ \mathrm{C} & 2.433980 & 0.149678 & -0.030013 \\ \mathrm{C} & 1.214913 & 0.097219 & -0.706026 \\ \mathrm{C} & -1.198384 & -0.105095 & 2.228249 \\ \mathrm{Pd} & -2.465050 & -1.784469 & 1.601119 \\ \mathrm{~S} & -0.531367 & -3.210429 & 2.450714 \\ \mathrm{C} & 0.418761 & -3.535784 & 0.933004 \\ \mathrm{C} & -2.455477 & 0.460435 & 1.946473 \\ \mathrm{C} & -2.820241 & 1.392668 & 0.805558 \\ \mathrm{O} & -4.201625 & 1.576149 & 0.928252 \\ \mathrm{C} & -4.504712 & 1.640916 & 2.301234 \\ \mathrm{C} & -3.440176 & 0.831624 & 3.005269 \\ \mathrm{C} & -3.373560 & 0.589169 & 4.315941 \\ \mathrm{~S} & -4.191749 & -3.151182 & 0.483224 \\ \mathrm{C} & -3.337728 & -3.519690 & -1.080642 \\ \mathrm{Pd} & -4.127000 & -5.139779 & 1.754664 \\ \mathrm{P} & -5.733195 & -4.235702 & 3.096060 \\ \mathrm{C} & -5.776396 & -2.421919 & 3.291678 \\ \mathrm{Pd} & -1.564624 & -5.265303 & 3.033198 \\ \mathrm{P} & -1.497150 & -4.569869 & 5.188948 \\ \mathrm{C} & 0.226472 & -4.409847 & 5.754017 \\ \mathrm{~S} & -3.423961 & -6.803613 & 3.385916 \\ \mathrm{C} & -3.071441 & -8.344942 & 2.473308 \\ \mathrm{O} & -1.061712 & -6.113281 & 1.149556 \\ \mathrm{C} & -1.864389 & -6.380759 & 0.231872 \\ \mathrm{O} & -3.091073 & -6.138434 & 0.194325 \\ \mathrm{C} & -7.349932 & -4.631263 & 2.357074 \\ \mathrm{C} & -5.863708 & -4.835775 & 4.812901 \\ \mathrm{C} & -2.225247 & -2.928542 & 5.508457 \\ \mathrm{C} & -2.249369 & -5.670885 & 6.430317\end{array}$




$\begin{array}{rrrr}\text { H } & -3.578660 & -0.764290 & 1.173606 \\ \mathrm{H} & -1.439988 & -6.899482 & -0.658543 \\ \mathrm{H} & -3.352803 & -2.596121 & -1.679197 \\ \mathrm{H} & -3.877058 & -4.314837 & -1.612982 \\ \mathrm{H} & -2.297821 & -3.832689 & -0.912200 \\ \mathrm{H} & -0.231656 & -3.859234 & 0.108738 \\ \mathrm{H} & 1.169357 & -4.314412 & 1.125832 \\ \mathrm{H} & 0.923083 & -2.595909 & 0.661401 \\ \mathrm{H} & -3.453809 & -8.276961 & 1.446065 \\ \mathrm{H} & -3.571022 & -9.170107 & 3.000155 \\ \mathrm{H} & -1.988135 & -8.528019 & 2.450956 \\ \mathrm{H} & -6.762311 & -4.406771 & 5.286148 \\ \mathrm{H} & -5.924599 & -5.933868 & 4.832793 \\ \mathrm{H} & -4.978125 & -4.525552 & 5.389724 \\ \mathrm{H} & -8.168738 & -4.231132 & 2.977824 \\ \mathrm{H} & -7.400529 & -4.189226 & 1.349924 \\ \mathrm{H} & -7.459872 & -5.722257 & 2.263836 \\ \mathrm{H} & -6.634141 & -2.143649 & 3.926205 \\ \mathrm{H} & -4.843615 & -2.051902 & 3.749171 \\ \mathrm{H} & -5.887133 & -1.942279 & 2.306320 \\ \mathrm{H} & -2.123706 & -5.241738 & 7.438175 \\ \mathrm{H} & -3.316660 & -5.837530 & 6.223818 \\ \mathrm{H} & -1.748256 & -6.650228 & 6.386950 \\ \mathrm{H} & -3.301208 & -2.932184 & 5.270364 \\ \mathrm{H} & -2.087296 & -2.637895 & 6.563031 \\ \mathrm{H} & -1.731157 & -2.190019 & 4.856160 \\ \mathrm{H} & 0.738066 & -5.378043 & 5.642257 \\ \mathrm{H} & 0.746190 & -3.667497 & 5.130031 \\ \mathrm{H} & 0.257294 & -4.102760 & 6.812631 \\ \mathrm{H} & -0.995755 & -0.288955 & 3.293028 \\ \mathrm{H} & -2.544298 & 0.031286 & 4.763455 \\ \mathrm{H} & -4.141782 & 0.964557 & 4.998968 \\ \mathrm{H} & -5.524756 & 1.252524 & 2.461347 \\ \mathrm{H} & -4.487178 & 2.689681 & 2.664242 \\ \mathrm{H} & -2.260454 & 2.344259 & 0.957555 \\ \mathrm{H} & -2.600390 & 1.028397 & -0.205617 \\ \mathrm{H} & 1.276777 & -0.022286 & 3.165511 \\ \mathrm{H} & 3.400965 & 0.139780 & 1.901458 \\ \mathrm{H} & 3.370211 & 0.219415 & -0.590089 \\ \mathrm{H} & 1.193923 & 0.117097 & -1.798867 \\ \mathrm{H} & -0.918303 & -0.061768 & -0.553206 \\ & & & \end{array}$

\begin{tabular}{|c|c|c|c|}
\hline \multicolumn{4}{|l|}{ XI } \\
\hline \multicolumn{4}{|c|}{ scf done: -3347.502706} \\
\hline $\mathrm{Pd}$ & -1.294381 & 6.963068 & 2.814363 \\
\hline $\mathrm{Pd}$ & 0.929778 & 5.791247 & 1.126305 \\
\hline $\mathrm{Pd}$ & -1.584540 & 4.022995 & 1.656377 \\
\hline $\mathrm{H}$ & -0.563663 & 5.005506 & 2.351144 \\
\hline $\mathrm{S}$ & 0.908094 & 7.884086 & 2.372690 \\
\hline S & 0.450165 & 3.581455 & 0.349920 \\
\hline $\mathrm{S}$ & -3.052308 & 5.357483 & 3.129219 \\
\hline $\mathrm{P}$ & -1.011303 & 6.928939 & 5.071463 \\
\hline $\mathrm{P}$ & 2.600663 & 4.831517 & 2.350384 \\
\hline $\mathrm{O}$ & -0.284428 & 6.659760 & -0.426118 \\
\hline $\mathrm{C}$ & -1.395939 & 7.206522 & -0.254576 \\
\hline $\mathrm{O}$ & -1.994821 & 7.423200 & 0.820609 \\
\hline $\mathrm{C}$ & 0.025466 & 3.786834 & -1.406729 \\
\hline
\end{tabular}




\begin{tabular}{|c|c|c|c|}
\hline $\mathrm{C}$ & -4.421190 & 5.981286 & 2.104921 \\
\hline $\mathrm{C}$ & -3.410538 & 2.753241 & 0.570700 \\
\hline $\mathrm{C}$ & -3.354663 & 2.983475 & -0.878033 \\
\hline $\mathrm{C}$ & -3.616728 & 4.270936 & -1.375754 \\
\hline $\mathrm{C}$ & -2.704388 & 1.826968 & 1.284664 \\
\hline $\mathrm{C}$ & -1.634530 & 0.879212 & 0.802307 \\
\hline $\mathrm{O}$ & -0.858892 & 0.616729 & 1.941830 \\
\hline C & -1.739729 & 0.545810 & 3.037598 \\
\hline $\mathrm{C}$ & -2.879073 & 1.483385 & 2.709506 \\
\hline $\mathrm{C}$ & -3.862412 & 1.857967 & 3.535025 \\
\hline $\mathrm{C}$ & -3.566853 & 4.532447 & -2.739684 \\
\hline $\mathrm{C}$ & -3.273897 & 3.505537 & -3.637764 \\
\hline $\mathrm{C}$ & -3.041378 & 2.217006 & -3.161024 \\
\hline $\mathrm{C}$ & -3.079877 & 1.956468 & -1.793761 \\
\hline C & 0.701389 & 9.222920 & 1.149615 \\
\hline C & 3.318482 & 5.853645 & 3.678549 \\
\hline $\mathrm{C}$ & 2.155433 & 3.283339 & 3.204592 \\
\hline $\mathrm{C}$ & 4.024628 & 4.387357 & 1.308279 \\
\hline $\mathrm{C}$ & 0.267935 & 8.033245 & 5.751964 \\
\hline $\mathrm{C}$ & -2.514260 & 7.410816 & 5.978960 \\
\hline $\mathrm{C}$ & -0.581106 & 5.282261 & 5.726265 \\
\hline $\mathrm{H}$ & -1.917445 & 7.548171 & -1.181134 \\
\hline $\mathrm{H}$ & -0.556300 & 2.907629 & -1.723523 \\
\hline $\mathrm{H}$ & 0.958575 & 3.835541 & -1.985819 \\
\hline $\mathrm{H}$ & -0.560943 & 4.699763 & -1.569831 \\
\hline $\mathrm{H}$ & -4.033524 & 6.452475 & 1.190949 \\
\hline $\mathrm{H}$ & -4.988997 & 6.722335 & 2.685668 \\
\hline $\mathrm{H}$ & -5.092137 & 5.143773 & 1.856197 \\
\hline $\mathrm{H}$ & 0.768651 & 8.823484 & 0.129218 \\
\hline $\mathrm{H}$ & 1.506500 & 9.952567 & 1.316130 \\
\hline $\mathrm{H}$ & -0.272273 & 9.712452 & 1.291640 \\
\hline $\mathrm{H}$ & 4.123391 & 5.293532 & 4.182463 \\
\hline $\mathrm{H}$ & 3.723768 & 6.789950 & 3.266709 \\
\hline $\mathrm{H}$ & 2.543930 & 6.112300 & 4.418168 \\
\hline $\mathrm{H}$ & 4.830530 & 3.955489 & 1.924720 \\
\hline $\mathrm{H}$ & 3.704356 & 3.653091 & 0.553951 \\
\hline $\mathrm{H}$ & 4.400215 & 5.281941 & 0.789081 \\
\hline $\mathrm{H}$ & 3.035199 & 2.891967 & 3.741307 \\
\hline $\mathrm{H}$ & 1.341127 & 3.465216 & 3.923841 \\
\hline $\mathrm{H}$ & 1.806873 & 2.531699 & 2.479067 \\
\hline $\mathrm{H}$ & 0.300715 & 7.932823 & 6.849398 \\
\hline $\mathrm{H}$ & 1.255739 & 7.800527 & 5.327076 \\
\hline $\mathrm{H}$ & 0.029631 & 9.075366 & 5.489263 \\
\hline $\mathrm{H}$ & 0.431839 & 5.008385 & 5.389530 \\
\hline $\mathrm{H}$ & -0.599760 & 5.287778 & 6.828443 \\
\hline $\mathrm{H}$ & -1.294514 & 4.529938 & 5.351120 \\
\hline $\mathrm{H}$ & -2.843592 & 8.407307 & 5.647367 \\
\hline $\mathrm{H}$ & -3.315496 & 6.687691 & 5.767224 \\
\hline $\mathrm{H}$ & -2.310311 & 7.437035 & 7.062254 \\
\hline $\mathrm{H}$ & -4.187696 & 3.315397 & 1.104964 \\
\hline $\mathrm{H}$ & -4.680770 & 2.505748 & 3.205332 \\
\hline $\mathrm{H}$ & -3.891403 & 1.517222 & 4.574398 \\
\hline $\mathrm{H}$ & -1.196900 & 0.822795 & 3.956799 \\
\hline $\mathrm{H}$ & -2.122209 & -0.488638 & 3.171586 \\
\hline $\mathrm{H}$ & -2.105816 & -0.055447 & 0.419293 \\
\hline $\mathrm{H}$ & -0.993273 & 1.286446 & 0.004360 \\
\hline $\mathrm{H}$ & -3.855603 & 5.075590 & -0.670026 \\
\hline $\mathrm{H}$ & -3.767794 & 5.542212 & -3.109110 \\
\hline $\mathrm{H}$ & -3.244696 & 3.706677 & -4.711912 \\
\hline $\mathrm{H}$ & -2.840614 & 1.402569 & -3.862487 \\
\hline
\end{tabular}


H $\quad-2.934043 \quad 0.932022 \quad-1.438822$

\section{Lacvp* (CPCM=toluene $)$}

\section{$\mathrm{HCOOH}$}

$$
5
$$

scf done: -189.658285

$\begin{array}{llll}\mathrm{C} & 5.382126 & -1.326025 & 2.548667 \\ \mathrm{O} & 4.761186 & -0.959054 & 3.504179 \\ \mathrm{O} & 5.490263 & -2.619377 & 2.215253 \\ \mathrm{H} & 5.919104 & -0.643441 & 1.859550 \\ \mathrm{H} & 6.027316 & -2.713929 & 1.412725\end{array}$

\section{$\mathrm{PMe}_{3}$}

13

scf done: -460.950768

$\begin{array}{lrrr}\mathrm{C} & 2.318248 & 5.822234 & 3.089836 \\ \mathrm{P} & 2.591247 & 5.573756 & 1.271554 \\ \mathrm{C} & 1.093621 & 6.476352 & 0.651096 \\ \mathrm{C} & 3.845095 & 6.914677 & 1.002027 \\ \mathrm{H} & 3.513145 & 7.874066 & 1.425072 \\ \mathrm{H} & 4.797612 & 6.635687 & 1.469149 \\ \mathrm{H} & 4.024931 & 7.048840 & -0.071723 \\ \mathrm{H} & 2.117264 & 6.876339 & 3.331476 \\ \mathrm{H} & 1.467149 & 5.217184 & 3.425744 \\ \mathrm{H} & 3.203363 & 5.496588 & 3.650012 \\ \mathrm{H} & 1.000064 & 7.473235 & 1.106434 \\ \mathrm{H} & 1.150725 & 6.592181 & -0.438169 \\ \mathrm{H} & 0.188762 & 5.899979 & 0.879984\end{array}$

\section{Enyne 1}

$$
25
$$

scf done: -539.198266

$\begin{array}{cccc}\mathrm{C} & 1.525646 & 3.876750 & -2.373576 \\ \mathrm{C} & 2.187422 & 3.079636 & -3.318777 \\ \mathrm{C} & 3.397600 & 3.545667 & -3.847336 \\ \mathrm{C} & 3.924180 & 4.772886 & -3.462439 \\ \mathrm{C} & 3.250976 & 5.557410 & -2.531254 \\ \mathrm{C} & 2.050932 & 5.101829 & -1.987235 \\ \mathrm{C} & 1.675218 & 1.779876 & -3.751347 \\ \mathrm{C} & 0.457471 & 1.279150 & -3.516157 \\ \mathrm{C} & 0.034861 & -0.083304 & -3.949985 \\ \mathrm{O} & -0.400187 & -0.900806 & -2.875981 \\ \mathrm{C} & 0.601065 & -1.150271 & -1.917438 \\ \mathrm{C} & 0.719330 & -0.111480 & -0.891621 \\ \mathrm{C} & 0.816081 & 0.719151 & -0.019634 \\ \mathrm{H} & 0.911991 & 1.464342 & 0.741718 \\ \mathrm{H} & -0.827769 & -0.038047 & -4.628754 \\ \mathrm{H} & 0.863091 & -0.579537 & -4.490982 \\ \mathrm{H} & 1.581091 & -1.295476 & -2.408893 \\ \mathrm{H} & 0.338651 & -2.103831 & -1.438981 \\ \mathrm{H} & -0.288444 & 1.850112 & -2.958865 \\ \mathrm{H} & 2.380357 & 1.168211 & -4.324669\end{array}$




$\begin{array}{llll}\mathrm{H} & 3.927202 & 2.930396 & -4.575742 \\ \mathrm{H} & 4.865113 & 5.117098 & -3.888881 \\ \mathrm{H} & 3.661351 & 6.517548 & -2.223116 \\ \mathrm{H} & 1.523844 & 5.705252 & -1.249670 \\ \mathrm{H} & 0.596935 & 3.523191 & -1.926690\end{array}$

Diene 3

25

scf done: -539.260066

C $\quad-3.581776 \quad 2.392893 \quad-1.771734$

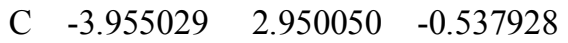

$\begin{array}{llll}\text { C } & -4.659796 & 4.165065 & -0.551578\end{array}$

$\begin{array}{llll}\text { C } & -4.947116 & 4.817099 & -1.741866\end{array}$

$\begin{array}{llll}\text { C } & -4.549611 & 4.261027 & -2.955627\end{array}$

$\begin{array}{llll}\text { C } & -3.873991 & 3.043678 & -2.963890\end{array}$

$\begin{array}{llll}\text { C } & -3.662106 & 2.334535 & 0.750005\end{array}$

$\begin{array}{llll}\text { C } & -2.712829 & 1.430305 & 1.051991\end{array}$

$\begin{array}{llll}\text { C } & -1.653044 & 0.844952 & 0.154740\end{array}$

$\begin{array}{llll}\mathrm{O} & -0.631380 & 0.393251 & 1.019855\end{array}$

$\begin{array}{llll}\text { C } & -1.248393 & -0.022269 & 2.221324\end{array}$

$\begin{array}{llll}\text { C } & -2.459878 & 0.860763 & 2.381634\end{array}$

$\begin{array}{llll}\text { C } & -3.137913 & 1.045747 & 3.516886\end{array}$

$\begin{array}{llll}\mathrm{H} & -4.279567 & 2.696927 & 1.577090\end{array}$

$\mathrm{H} \quad-4.024352 \quad 1.676300 \quad 3.568315$

$\mathrm{H} \quad-2.829436 \quad 0.565199 \quad 4.443929$

$\mathrm{H} \quad-0.522267 \quad 0.073380 \quad 3.037840$

$\mathrm{H} \quad-1.550603 \quad-1.085107 \quad 2.156505$

$\mathrm{H} \quad-2.054873 \quad-0.000070 \quad-0.440140$

$\begin{array}{llll}\mathrm{H} & -1.219322 & 1.571965 & -0.545289\end{array}$

$\begin{array}{llll}\mathrm{H} & -4.973293 & 4.600867 & 0.397593\end{array}$

$\mathrm{H} \quad-5.487427 \quad 5.762243 \quad-1.724384$

$\mathrm{H} \quad-4.776058 \quad 4.768347 \quad-3.891765$

$\mathrm{H} \quad-3.578222 \quad 2.590790 \quad-3.908905$

$\mathrm{H} \quad-3.090746 \quad 1.422222 \quad-1.805415$

\section{A}

57

scf done: -3077.372050

$\begin{array}{llll}\text { C } & 7.183377 & 1.986196 & -2.004257\end{array}$

$\begin{array}{llll}\text { P } & 7.048171 & 0.827065 & -0.598742\end{array}$

$\begin{array}{llll}\text { C } & 7.178369 & -0.816568 & -1.388263\end{array}$

$\begin{array}{llll}\mathrm{Pd} & 8.636328 & 1.407271 & 1.003922\end{array}$

$\begin{array}{llll}\mathrm{Pd} & 11.425800 & 1.400337 & 1.916648\end{array}$

$\begin{array}{llll}\mathrm{P} & 13.648279 & 0.828311 & 1.511107\end{array}$

$\begin{array}{llll}\text { C } & 14.857451 & 0.964765 & 2.876608\end{array}$

$\begin{array}{llll}\text { C } & 5.280200 & 0.921292 & -0.142238\end{array}$

$\begin{array}{llll}\text { S } & 10.441509 & 0.147016 & 0.187744\end{array}$

$\begin{array}{llll}\text { C } & 10.194268 & -1.506819 & 0.944259\end{array}$

$\begin{array}{llll}\mathrm{Pd} & 9.481125 & 3.176964 & 3.191766\end{array}$

$\begin{array}{llll}\text { S } & 11.775728 & 2.953840 & 3.643689\end{array}$

$\begin{array}{llll}\text { C } & 11.815690 & 1.914197 & 5.155475\end{array}$

$\begin{array}{llll}\text { S } & 7.362008 & 2.982117 & 2.194593\end{array}$

$\begin{array}{llll}\text { C } & 6.417719 & 1.955695 & 3.388061\end{array}$

$\begin{array}{llll}\text { P } & 9.052467 & 5.047520 & 4.516470\end{array}$

$\begin{array}{llll}\text { C } & 14.019913 & -0.814835 & 0.800958\end{array}$

$\begin{array}{llll}\text { C } & 14.298431 & 1.987833 & 0.257937\end{array}$ 


$\begin{array}{cccc}\text { H } & 11.624928 & 2.551470 & 6.025833 \\ \mathrm{H} & 12.814457 & 1.475240 & 5.256092 \\ \mathrm{H} & 11.067364 & 1.118436 & 5.110682 \\ \mathrm{H} & 5.498394 & 1.607082 & 2.904894 \\ \mathrm{H} & 6.143624 & 2.574668 & 4.249333 \\ \mathrm{H} & 7.005249 & 1.097670 & 3.725482 \\ \mathrm{H} & 11.139483 & -2.059472 & 0.903492 \\ \mathrm{H} & 9.445407 & -2.053700 & 0.360544 \\ \mathrm{H} & 9.861699 & -1.423946 & 1.982428 \\ \mathrm{H} & 15.089710 & -0.891651 & 0.563808 \\ \mathrm{H} & 13.758335 & -1.604237 & 1.515094 \\ \mathrm{H} & 13.437986 & -0.960891 & -0.117095 \\ \mathrm{H} & 15.874738 & 0.796735 & 2.497240 \\ \mathrm{H} & 14.801327 & 1.965004 & 3.322694 \\ \mathrm{H} & 14.641034 & 0.218638 & 3.650022 \\ \mathrm{H} & 15.348225 & 1.763479 & 0.024390 \\ \mathrm{H} & 13.697385 & 1.912175 & -0.656127 \\ \mathrm{H} & 14.221705 & 3.013010 & 0.639626 \\ \mathrm{H} & 4.649932 & 0.681779 & -1.009570 \\ \mathrm{H} & 5.059624 & 0.210404 & 0.662737 \\ \mathrm{H} & 5.043351 & 1.934440 & 0.204131 \\ \mathrm{H} & 7.021718 & -1.604845 & -0.642601 \\ \mathrm{H} & 6.422767 & -0.919799 & -2.178706 \\ \mathrm{H} & 8.176240 & -0.937595 & -1.827005 \\ \mathrm{H} & 7.025029 & 3.010599 & -1.646199 \\ \mathrm{H} & 8.189087 & 1.921066 & -2.436489 \\ \mathrm{H} & 6.439203 & 1.750980 & -2.777301 \\ \mathrm{C} & 10.084049 & 5.327009 & 6.000298 \\ \mathrm{C} & 9.368341 & 6.533193 & 3.500729 \\ \mathrm{C} & 7.354587 & 5.317473 & 5.141051 \\ \mathrm{H} & 7.283503 & 6.294476 & 5.638231 \\ \mathrm{H} & 6.645078 & 5.289728 & 4.305360 \\ \mathrm{H} & 7.086359 & 4.534910 & 5.860377 \\ \mathrm{H} & 9.175799 & 7.449508 & 4.075374 \\ \mathrm{H} & 10.411758 & 6.528371 & 3.163436 \\ \mathrm{H} & 8.718678 & 6.515107 & 2.617521 \\ \mathrm{H} & 9.838772 & 6.295546 & 6.456940 \\ \mathrm{H} & 9.912043 & 4.535409 & 6.738804 \\ \mathrm{H} & 11.143307 & 5.322680 & 5.716446\end{array}$

I

62

scf done: -3267.051668

$\begin{array}{llll}\mathrm{C} & 7.939144 & 1.861597 & -2.127707 \\ \mathrm{P} & 7.128954 & 1.414659 & -0.555023\end{array}$

$\begin{array}{llll}\mathrm{P} & 7.128954 & 1.414659 & -0.555023\end{array}$

$\begin{array}{llll}\mathrm{Pd} & 6.819605 & -0.901101 & -0.433866\end{array}$

$\begin{array}{llll}\mathrm{S} & 4.810395 & -1.035287 & -1.661994\end{array}$

$\begin{array}{llll}\text { C } & 3.427150 & -0.554020 & -0.556259\end{array}$

$\begin{array}{llll}\text { C } & 8.179195 & 2.189368 & 0.723955\end{array}$

$\begin{array}{llll}\text { C } & 5.627785 & 2.455925 & -0.523380\end{array}$

$\begin{array}{llll}\mathrm{Pd} & 7.829532 & -3.550749 & 0.281277\end{array}$

$\begin{array}{llll}\text { S } & 6.303767 & -5.225891 & -0.326821\end{array}$

$\begin{array}{llll}\text { C } & 5.420351 & -5.673896 & 1.217290\end{array}$

$\begin{array}{llll}\text { S } & 8.895731 & -1.469643 & 0.526349\end{array}$

$\begin{array}{llll}\text { C } & 8.789153 & -1.119846 & 2.324551\end{array}$

$\begin{array}{llll}\mathrm{Pd} & 5.247695 & -3.279077 & -1.099168\end{array}$

$\begin{array}{llll}\mathrm{P} & 3.322805 & -4.258803 & -1.984341\end{array}$

$\begin{array}{llll}\text { C } & 1.892036 & -4.198929 & -0.847154\end{array}$ 


$\begin{array}{cccc}\mathrm{P} & 9.371917 & -4.896278 & 1.398124 \\ \mathrm{C} & 2.692255 & -3.502696 & -3.523384 \\ \mathrm{C} & 3.446293 & -6.030638 & -2.414367 \\ \mathrm{O} & 5.430996 & -2.304249 & 2.317411 \\ \mathrm{C} & 5.205305 & -1.033543 & 2.677968 \\ \mathrm{O} & 4.660441 & -0.732447 & 3.701650 \\ \mathrm{H} & 6.032585 & -6.393238 & 1.774256 \\ \mathrm{H} & 4.478654 & -6.166683 & 0.948218 \\ \mathrm{H} & 5.215010 & -4.799330 & 1.841931 \\ \mathrm{H} & 8.701417 & -0.038458 & 2.477107 \\ \mathrm{H} & 9.716634 & -1.459782 & 2.800277 \\ \mathrm{H} & 7.935160 & -1.625984 & 2.784746 \\ \mathrm{H} & 2.492237 & -0.616598 & -1.125223 \\ \mathrm{H} & 3.561985 & 0.484010 & -0.232974 \\ \mathrm{H} & 3.367116 & -1.203661 & 0.322092 \\ \mathrm{H} & 1.020191 & -4.683677 & -1.307586 \\ \mathrm{H} & 2.136283 & -4.714191 & 0.089321 \\ \mathrm{H} & 1.639166 & -3.159062 & -0.610366 \\ \mathrm{H} & 2.492773 & -6.390427 & -2.824017 \\ \mathrm{H} & 4.238463 & -6.177813 & -3.157201 \\ \mathrm{H} & 3.699454 & -6.616140 & -1.522743 \\ \mathrm{H} & 1.790642 & -4.028499 & -3.865670 \\ \mathrm{H} & 2.454763 & -2.446311 & -3.353373 \\ \mathrm{H} & 3.461525 & -3.558611 & -4.302134 \\ \mathrm{H} & 8.280705 & 3.265897 & 0.531988 \\ \mathrm{H} & 7.724380 & 2.051048 & 1.713231 \\ \mathrm{H} & 9.175029 & 1.730189 & 0.720509 \\ \mathrm{H} & 5.090518 & 2.309646 & 0.422340 \\ \mathrm{H} & 5.898245 & 3.516618 & -0.614318 \\ \mathrm{H} & 4.968962 & 2.183649 & -1.356798 \\ \mathrm{H} & 8.912930 & 1.361952 & -2.192184 \\ \mathrm{H} & 7.318066 & 1.522216 & -2.965327 \\ \mathrm{H} & 8.082081 & 2.948480 & -2.197376 \\ \mathrm{H} & 5.580974 & -0.296131 & 1.929386 \\ \mathrm{H} & 5.876500 & -2.332453 & 1.432985 \\ \mathrm{C} & 9.455354 & -6.647424 & 0.882603 \\ \mathrm{C} & 11.116023 & -4.357488 & 1.319693 \\ \mathrm{C} & 9.012484 & -4.986919 & 3.189235 \\ \mathrm{H} & 9.728265 & -5.649584 & 3.694939 \\ \mathrm{H} & 9.073350 & -3.987819 & 3.636243 \\ \mathrm{H} & 7.996405 & -5.368090 & 3.346590 \\ \mathrm{H} & 11.756016 & -5.043868 & 1.890071 \\ \mathrm{H} & 11.447197 & -4.337937 & 0.275251 \\ \mathrm{H} & 11.216281 & -3.345705 & 1.728771 \\ \mathrm{H} & 10.215633 & -7.182498 & 1.467351 \\ \mathrm{H} & 8.481625 & -7.128758 & 1.030031 \\ \mathrm{H} & 9.708964 & -6.707524 & -0.181786\end{array}$

\section{TS (I-IV)}

62

scf done: -3267.007905

$\begin{array}{cccc}\mathrm{C} & 0.419603 & 0.846622 & -0.408106 \\ \mathrm{P} & 0.351220 & 0.694993 & 1.410134 \\ \mathrm{C} & 2.056971 & 1.021472 & 1.963359 \\ \mathrm{Pd} & -1.171492 & 2.106987 & 2.408958 \\ \mathrm{~S} & -2.482002 & 0.272422 & 3.145545 \\ \mathrm{C} & -3.497509 & 0.902450 & 4.534828 \\ \mathrm{~S} & -0.320161 & 3.849207 & 1.039668\end{array}$




$\begin{array}{cccc}\mathrm{C} & -0.687268 & 5.413945 & 1.916711 \\ \mathrm{Pd} & -2.409423 & 3.261291 & -0.112527 \\ \mathrm{~S} & -4.320051 & 2.110565 & -0.855143 \\ \mathrm{C} & -5.715906 & 3.195219 & -0.368943 \\ \mathrm{P} & -1.910061 & 4.568873 & -1.997668 \\ \mathrm{C} & -0.729422 & 3.732728 & -3.114875 \\ \mathrm{C} & -3.324898 & 4.977241 & -3.080075 \\ \mathrm{C} & -1.117081 & 6.188389 & -1.697628 \\ \mathrm{Pd} & -3.699764 & 1.021374 & 1.140629 \\ \mathrm{P} & -5.015704 & -0.929823 & 1.101463 \\ \mathrm{C} & -6.314492 & -1.030211 & -0.180982 \\ \mathrm{O} & -3.955663 & 3.798943 & 2.521286 \\ \mathrm{C} & -3.237753 & 4.007620 & 3.533922 \\ \mathrm{O} & -2.115022 & 3.513301 & 3.797701 \\ \mathrm{C} & 0.128619 & -1.097891 & 1.658297 \\ \mathrm{C} & -4.035663 & -2.450144 & 0.832595 \\ \mathrm{C} & -5.916640 & -1.284803 & 2.652511 \\ \mathrm{H} & -3.226654 & 2.838518 & 1.597737 \\ \mathrm{H} & -0.168351 & 6.221845 & 1.389026 \\ \mathrm{H} & -0.317620 & 5.348532 & 2.943569 \\ \mathrm{H} & -1.762759 & 5.612480 & 1.926763 \\ \mathrm{H} & -5.540978 & 3.644291 & 0.612545 \\ \mathrm{H} & -6.631579 & 2.593993 & -0.351197 \\ \mathrm{H} & -5.822989 & 3.978119 & -1.128699 \\ \mathrm{H} & -2.861617 & 1.472941 & 5.217312 \\ \mathrm{H} & -3.915337 & 0.037078 & 5.060042 \\ \mathrm{H} & -4.308331 & 1.540441 & 4.169780 \\ \mathrm{H} & 0.946494 & -1.634223 & 1.158785 \\ \mathrm{H} & 0.131890 & -1.342700 & 2.725778 \\ \mathrm{H} & -0.826374 & -1.421974 & 1.225948 \\ \mathrm{H} & 2.761080 & 0.348745 & 1.455421 \\ \mathrm{H} & 2.316753 & 2.062210 & 1.738905 \\ \mathrm{H} & 2.127260 & 0.870740 & 3.046161 \\ \mathrm{H} & 1.149078 & 0.135209 & -0.817860 \\ \mathrm{H} & -0.572165 & 0.638665 & -0.830087 \\ \mathrm{H} & 0.714418 & 1.864171 & -0.690653 \\ \mathrm{H} & -6.567414 & -2.160718 & 2.529276 \\ \mathrm{H} & -5.194413 & -1.488544 & 3.451958 \\ \mathrm{H} & -6.525117 & -0.420581 & 2.942603 \\ \mathrm{H} & -3.295282 & -2.549643 & 1.635842 \\ \mathrm{H} & -4.683578 & -3.337078 & 0.829303 \\ \mathrm{H} & -3.508228 & -2.388413 & -0.126658 \\ \mathrm{H} & -7.048606 & -0.230203 & -0.029873 \\ \mathrm{H} & -5.862888 & -0.904331 & -1.171833 \\ \mathrm{H} & -6.827872 & -2.000159 & -0.135051 \\ \mathrm{H} & -3.645526 & 4.715249 & 4.284479 \\ \mathrm{H} & -0.954990 & 6.716012 & -2.647324 \\ \mathrm{H} & -1.747203 & 6.805313 & -1.046424 \\ \mathrm{H} & -0.148407 & 6.033336 & -1.206943 \\ \mathrm{H} & -2.992695 & 5.548000 & -3.957444 \\ \mathrm{H} & -3.810663 & 4.051726 & -3.412009 \\ \mathrm{H} & -4.056179 & 5.575768 & -2.523908 \\ \mathrm{H} & -0.517836 & 4.353683 & -3.995964 \\ \mathrm{H} & 0.207663 & 3.547053 & -2.575440 \\ \mathrm{H} & -1.142071 & 2.770644 & -3.441555\end{array}$

\section{IV}

62 
scf done: -3267.013703

\begin{tabular}{|c|c|c|c|}
\hline $\mathrm{C}$ & 1.411383 & 1.423222 & -0.045263 \\
\hline 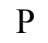 & 0.355022 & 0.762573 & 1.287562 \\
\hline $\mathrm{C}$ & 1.527403 & 0.034471 & 2.478151 \\
\hline $\mathrm{Pd}$ & -1.092186 & 2.236639 & 2.340393 \\
\hline S & -2.307552 & 0.334386 & 3.156272 \\
\hline $\mathrm{C}$ & -3.233102 & 0.929690 & 4.620719 \\
\hline S & -0.372979 & 4.010516 & 0.905384 \\
\hline $\mathrm{C}$ & -0.920845 & 5.549419 & 1.730485 \\
\hline $\mathrm{Pd}$ & -2.422008 & 3.233303 & -0.220536 \\
\hline S & -4.412699 & 2.061277 & -0.774892 \\
\hline $\mathrm{C}$ & -5.673716 & 3.223891 & -0.120620 \\
\hline $\mathrm{P}$ & -1.979218 & 4.436188 & -2.167753 \\
\hline $\mathrm{C}$ & -0.443527 & 3.925889 & -3.013593 \\
\hline $\mathrm{C}$ & -3.255064 & 4.354957 & -3.470745 \\
\hline $\mathrm{C}$ & -1.738394 & 6.225710 & -1.899993 \\
\hline $\mathrm{Pd}$ & -3.668775 & 1.016382 & 1.222244 \\
\hline $\mathrm{P}$ & -4.995423 & -0.907473 & 1.275620 \\
\hline $\mathrm{C}$ & -6.455650 & -0.904719 & 0.179418 \\
\hline $\mathrm{O}$ & -3.855436 & 4.099429 & 2.650743 \\
\hline $\mathrm{C}$ & -3.114146 & 4.104831 & 3.636256 \\
\hline $\mathrm{O}$ & -1.979554 & 3.538002 & 3.795704 \\
\hline $\mathrm{C}$ & -0.436493 & -0.678196 & 0.491843 \\
\hline $\mathrm{C}$ & -4.074560 & -2.407182 & 0.787266 \\
\hline $\mathrm{C}$ & -5.679081 & -1.334717 & 2.913830 \\
\hline $\mathrm{H}$ & -2.848063 & 2.582914 & 1.372922 \\
\hline $\mathrm{H}$ & -0.516222 & 6.396928 & 1.166784 \\
\hline $\mathrm{H}$ & -0.521739 & 5.564197 & 2.748540 \\
\hline $\mathrm{H}$ & -2.012579 & 5.601014 & 1.766014 \\
\hline $\mathrm{H}$ & -5.328067 & 3.677768 & 0.813354 \\
\hline $\mathrm{H}$ & -6.603595 & 2.668915 & 0.046546 \\
\hline $\mathrm{H}$ & -5.855913 & 3.996083 & -0.876026 \\
\hline $\mathrm{H}$ & -2.590932 & 1.597485 & 5.200414 \\
\hline $\mathrm{H}$ & -3.507305 & 0.056615 & 5.222030 \\
\hline $\mathrm{H}$ & -4.132568 & 1.472676 & 4.314851 \\
\hline $\mathrm{H}$ & 0.329526 & -1.322495 & 0.040231 \\
\hline $\mathrm{H}$ & -0.996861 & -1.256265 & 1.235624 \\
\hline $\mathrm{H}$ & -1.132578 & -0.336816 & -0.285541 \\
\hline $\mathrm{H}$ & 2.199899 & -0.672702 & 1.973895 \\
\hline $\mathrm{H}$ & 2.119138 & 0.831737 & 2.941486 \\
\hline $\mathrm{H}$ & 0.969812 & -0.486691 & 3.265024 \\
\hline $\mathrm{H}$ & 2.071977 & 0.627064 & -0.412789 \\
\hline $\mathrm{H}$ & 0.785146 & 1.779266 & -0.872858 \\
\hline $\mathrm{H}$ & 2.016747 & 2.259453 & 0.320614 \\
\hline $\mathrm{H}$ & -6.334984 & -2.212180 & 2.836290 \\
\hline $\mathrm{H}$ & -4.859579 & -1.562138 & 3.605662 \\
\hline $\mathrm{H}$ & -6.252618 & -0.491471 & 3.315439 \\
\hline $\mathrm{H}$ & -3.232772 & -2.555681 & 1.474619 \\
\hline $\mathrm{H}$ & -4.726123 & -3.290647 & 0.821479 \\
\hline $\mathrm{H}$ & -3.681532 & -2.289082 & -0.229361 \\
\hline $\mathrm{H}$ & -7.142128 & -0.104192 & 0.478893 \\
\hline $\mathrm{H}$ & -6.140831 & -0.724105 & -0.854753 \\
\hline $\mathrm{H}$ & -6.981671 & -1.866677 & 0.239049 \\
\hline $\mathrm{H}$ & -3.428771 & 4.674805 & 4.541720 \\
\hline $\mathrm{H}$ & -1.613347 & 6.739712 & -2.862410 \\
\hline $\mathrm{H}$ & -2.598349 & 6.652696 & -1.371381 \\
\hline $\mathrm{H}$ & -0.838797 & 6.384081 & -1.294088 \\
\hline $\mathrm{H}$ & -2.946002 & 4.937582 & -4.348873 \\
\hline $\mathrm{H}$ & -3.419982 & 3.312127 & -3.764668 \\
\hline $\mathrm{H}$ & -4.198776 & 4.760125 & -3.086960 \\
\hline
\end{tabular}




$\begin{array}{rrrr}\mathrm{H} & -0.281552 & 4.535757 & -3.912405 \\ \mathrm{H} & 0.404407 & 4.060223 & -2.331108 \\ \mathrm{H} & -0.504464 & 2.870399 & -3.302779\end{array}$

\section{$\mathrm{V}$}

62

scf done: -3267.015256

$\begin{array}{llll}\mathrm{P} & -5.039948 & 1.662012 & 1.186557\end{array}$

$\begin{array}{llll}\mathrm{Pd} & -4.036394 & 3.261216 & -0.153354\end{array}$

$\begin{array}{llll}\text { S } & -3.568598 & 4.306250 & -2.346325\end{array}$

$\begin{array}{llll}\text { C } & -5.134482 & 5.134668 & -2.794985\end{array}$

$\begin{array}{llll}\mathrm{S} & -2.385511 & 3.920664 & 1.663764\end{array}$

$\begin{array}{llll}\mathrm{Pd} & -1.341880 & 5.899687 & 0.862670\end{array}$

$\begin{array}{llll}\mathrm{O} & -3.300307 & 6.913633 & 0.906416\end{array}$

$\begin{array}{llll}\text { C } & -3.929264 & 7.405967 & -0.058368\end{array}$

$\begin{array}{llll}\mathrm{O} & -3.662489 & 7.331297 & -1.283764\end{array}$

$\begin{array}{llll}\mathrm{Pd} & -2.044212 & 6.066707 & -1.904554\end{array}$

$\begin{array}{llll}\text { P } & -0.467829 & 4.748881 & -2.903686\end{array}$

$\begin{array}{llll}\text { C } & -0.488908 & 2.997157 & -2.391080\end{array}$

$\begin{array}{llll}\text { C } & -3.485277 & 4.631575 & 2.943310\end{array}$

$\begin{array}{llll}\text { S } & -0.402540 & 7.466099 & -0.746675\end{array}$

$\begin{array}{llll}\text { C } & -1.199838 & 9.091144 & -0.463313\end{array}$

$\begin{array}{llll}\mathrm{P} & 0.640593 & 5.080247 & 1.617106\end{array}$

$\begin{array}{llll}\text { C } & 1.160626 & 3.440456 & 1.009512\end{array}$

$\begin{array}{llll}\text { C } & 1.270610 & 5.260217 & -2.687500\end{array}$

$\begin{array}{llll}\text { C } & -0.683696 & 4.686307 & -4.711684\end{array}$

$\begin{array}{llll}\text { C } & 2.101623 & 6.139241 & 1.352479\end{array}$

$\begin{array}{llll}\mathrm{C} & 0.580850 & 4.877264 & 3.428475\end{array}$

$\begin{array}{llll}\mathrm{H} & -5.201309 & 2.706319 & -1.075247\end{array}$

$\begin{array}{llll}\mathrm{H} & -5.868110 & 4.349771 & -3.009116\end{array}$

$\begin{array}{llll}\mathrm{H} & -4.970747 & 5.741921 & -3.690577\end{array}$

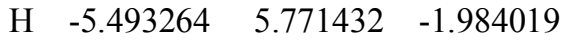

$\begin{array}{llll}\mathrm{H} & -4.056898 & 5.464230 & 2.524615\end{array}$

$\begin{array}{llll}\mathrm{H} & -2.876332 & 4.987172 & 3.782440\end{array}$

$\begin{array}{llll}\mathrm{H} & -4.165863 & 3.853365 & 3.308650\end{array}$

$\begin{array}{llll}\mathrm{H} & -1.988844 & 9.251795 & -1.203143\end{array}$

$\begin{array}{llll}\mathrm{H} & -0.431470 & 9.863391 & -0.565808\end{array}$

$\begin{array}{llll}\mathrm{H} & -1.628968 & 9.129259 & 0.541761\end{array}$

$\begin{array}{llll}\mathrm{H} & 1.930929 & 4.583333 & -3.245170\end{array}$

$\begin{array}{llll}\mathrm{H} & 1.405607 & 6.284173 & -3.053073\end{array}$

$\mathrm{H} \quad 1.547065 \quad 5.238163 \quad-1.626370$

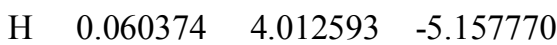

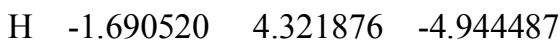

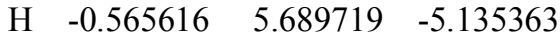

$\begin{array}{llll}\mathrm{H} & 0.363064 & 2.467351 & -2.837697\end{array}$

$\begin{array}{llll}\mathrm{H} & -0.443172 & 2.914026 & -1.298699\end{array}$

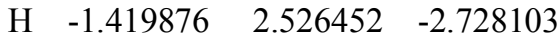

$\begin{array}{llll}\mathrm{H} & 2.980706 & 5.673783 & 1.817581\end{array}$

$\begin{array}{llll}\mathrm{H} & 2.291565 & 6.289172 & 0.283913\end{array}$

$\begin{array}{llll}\mathrm{H} & 1.929118 & 7.121666 & 1.806490\end{array}$

$\begin{array}{llll}\mathrm{H} & 1.407938 & 3.487766 & -0.058155\end{array}$

$\begin{array}{llll}\mathrm{H} & 2.050643 & 3.107700 & 1.560469\end{array}$

$\begin{array}{llll}\mathrm{H} & 0.351825 & 2.715336 & 1.160120\end{array}$

$\begin{array}{llll}\mathrm{H} & 0.323830 & 5.831838 & 3.901726\end{array}$

$\begin{array}{llll}\mathrm{H} & -0.184076 & 4.135633 & 3.685414\end{array}$

$\begin{array}{llll}\mathrm{H} & 1.557152 & 4.539521 & 3.802200\end{array}$

$\begin{array}{llll}\mathrm{H} & -4.833758 & 7.991099 & 0.197644\end{array}$

$\begin{array}{llll}\text { C } & -4.091098 & 0.983091 & 2.596754\end{array}$ 


$\begin{array}{rrrr}\text { C } & -6.632173 & 2.177362 & 1.917543 \\ \mathrm{C} & -5.476287 & 0.145402 & 0.264752 \\ \mathrm{H} & -7.072471 & 1.354935 & 2.497056 \\ \mathrm{H} & -6.494889 & 3.045784 & 2.571384 \\ \mathrm{H} & -7.319076 & 2.460937 & 1.111800 \\ \mathrm{H} & -4.661334 & 0.169203 & 3.064420 \\ \mathrm{H} & -3.132285 & 0.591015 & 2.238697 \\ \mathrm{H} & -3.887659 & 1.751203 & 3.350250 \\ \mathrm{H} & -5.927609 & -0.596661 & 0.936584 \\ \mathrm{H} & -6.183491 & 0.393136 & -0.534548 \\ \mathrm{H} & -4.576465 & -0.282096 & -0.192081\end{array}$

VI

74

scf done: -3345.251010

$\begin{array}{llll}\mathrm{C} & 1.050000 & -1.413862 & -0.165695 \\ \mathrm{P} & 0.566119 & -0.817115 & 1.483316\end{array}$

$\begin{array}{llll}\mathrm{P} & 0.566119 & -0.817115 & 1.483316\end{array}$

$\begin{array}{llll}\text { Pd } & 2.294932 & -0.015024 & 2.761331\end{array}$

$\begin{array}{llll}\text { Pd } & 4.789366 & 0.044692 & 1.060860\end{array}$

$\begin{array}{llll}\text { S } & 5.166665 & 2.176674 & 0.066695\end{array}$

$\begin{array}{llll}\mathrm{C} & 6.650240 & 2.820196 & 0.913367\end{array}$

$\begin{array}{llll}\text { C } & -0.830464 & 0.297115 & 1.129990\end{array}$

$\begin{array}{llll}\text { C } & -0.186585 & -2.258250 & 2.307020\end{array}$

$\begin{array}{llll}\mathrm{O} & 3.628607 & 0.482046 & 4.377575\end{array}$

$\begin{array}{llll}\text { C } & 4.836355 & 0.784959 & 4.194979\end{array}$

$\begin{array}{llll}\text { O } & 5.477476 & 0.825527 & 3.123038\end{array}$

$\begin{array}{llll}\mathrm{S} & 1.264594 & 2.155404 & 2.841786\end{array}$

$\begin{array}{llll}\text { C } & 1.610879 & 2.804454 & 4.513202\end{array}$

$\begin{array}{llll}\mathrm{Pd} & 3.232706 & 2.644004 & 1.481453\end{array}$

$\begin{array}{llll}\text { C } & 3.618159 & 4.844584 & 2.427101\end{array}$

$\begin{array}{llll}\text { S } & 3.685668 & -1.882312 & 2.046040\end{array}$

$\begin{array}{llll}\text { C } & 4.713845 & -2.385902 & 3.473568\end{array}$

$\begin{array}{llll}\mathrm{P} & 5.441987 & -1.129200 & -0.778849\end{array}$

$\begin{array}{llll}\text { C } & 6.160079 & -0.184505 & -2.162093\end{array}$

$\begin{array}{llll}\text { C } & 6.792344 & -2.239309 & -0.255598\end{array}$

$\begin{array}{llll}\text { C } & 4.249157 & -2.249888 & -1.581505\end{array}$

$\begin{array}{llll}\mathrm{H} & 2.678366 & 1.407399 & 0.659571\end{array}$

$\begin{array}{llll}\mathrm{H} & 5.124271 & -3.377751 & 3.257548\end{array}$

$\begin{array}{llll}\mathrm{H} & 4.089296 & -2.433975 & 4.370607\end{array}$

$\begin{array}{llll}\mathrm{H} & 5.528269 & -1.673889 & 3.630725\end{array}$

$\begin{array}{llll}\mathrm{H} & 6.730640 & 2.401565 & 1.920783\end{array}$

$\begin{array}{llll}\mathrm{H} & 6.585520 & 3.913045 & 0.954978\end{array}$

$\begin{array}{llll}\mathrm{H} & 7.530777 & 2.537422 & 0.325228\end{array}$

$\mathrm{H} \quad 2.532928 \quad 2.373155 \quad 4.911300$

$\begin{array}{llll}\mathrm{H} & 0.769461 & 2.535401 & 5.159827\end{array}$

$\begin{array}{llll}\mathrm{H} & 1.693147 & 3.895223 & 4.463575\end{array}$

$\begin{array}{llll}\mathrm{H} & -1.567203 & -0.236581 & 0.513676\end{array}$

$\begin{array}{llll}\mathrm{H} & -1.298208 & 0.634333 & 2.060739\end{array}$

$\begin{array}{llll}\mathrm{H} & -0.471529 & 1.174311 & 0.577416\end{array}$

$\begin{array}{llll}\mathrm{H} & -1.005628 & -2.660269 & 1.695699\end{array}$

$\begin{array}{llll}\mathrm{H} & 0.575408 & -3.032349 & 2.452319\end{array}$

$\mathrm{H} \quad-0.578178 \quad-1.961444 \quad 3.286376$

$\begin{array}{llll}\mathrm{H} & 0.171341 & -1.808899 & -0.693317\end{array}$

$\begin{array}{llll}\mathrm{H} & 1.468767 & -0.579036 & -0.742820\end{array}$

$\begin{array}{llll}\mathrm{H} & 1.799528 & -2.206851 & -0.069654\end{array}$

$\begin{array}{llll}\mathrm{H} & 5.393428 & 1.051086 & 5.117111\end{array}$

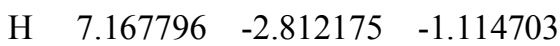

$\begin{array}{llll}\mathrm{H} & 7.611633 & -1.651961 & 0.174163\end{array}$ 


$\begin{array}{rrrr}\mathrm{H} & 6.420318 & -2.933253 & 0.507326 \\ \mathrm{H} & 6.520088 & -0.878818 & -2.933139 \\ \mathrm{H} & 5.413507 & 0.490119 & -2.596226 \\ \mathrm{H} & 6.995866 & 0.423690 & -1.797938 \\ \mathrm{H} & 4.755544 & -2.794116 & -2.390578 \\ \mathrm{H} & 3.870309 & -2.972180 & -0.849057 \\ \mathrm{H} & 3.408803 & -1.683526 & -2.001728 \\ \mathrm{C} & 4.436882 & 4.086610 & 2.924948 \\ \mathrm{C} & 2.687467 & 5.885333 & 1.974687 \\ \mathrm{O} & 2.794717 & 6.195598 & 0.613779 \\ \mathrm{C} & 1.771467 & 5.698482 & -0.230265 \\ \mathrm{C} & 2.065652 & 4.382261 & -0.872417 \\ \mathrm{C} & 1.117456 & 3.450844 & -1.054543 \\ \mathrm{C} & 1.207094 & 2.233150 & -1.862108 \\ \mathrm{C} & 0.023952 & 1.552280 & -2.183077 \\ \mathrm{H} & 5.221986 & 3.549404 & 3.421407 \\ \mathrm{C} & 0.033656 & 0.431131 & -3.005762 \\ \mathrm{C} & 1.235972 & -0.039590 & -3.523673 \\ \mathrm{C} & 2.423541 & 0.619791 & -3.203817 \\ \mathrm{C} & 2.415464 & 1.736421 & -2.376880 \\ \mathrm{H} & 1.660645 & 6.460993 & -1.015722 \\ \mathrm{H} & 0.811673 & 5.654070 & 0.315069 \\ \mathrm{H} & 1.655008 & 5.592687 & 2.235781 \\ \mathrm{H} & 2.917530 & 6.797852 & 2.545016 \\ \mathrm{H} & 3.057038 & 4.280719 & -1.321107 \\ \mathrm{H} & 0.133311 & 3.632746 & -0.606500 \\ \mathrm{H} & -0.923876 & 1.934525 & -1.800540 \\ \mathrm{H} & -0.902003 & -0.069125 & -3.250217 \\ \mathrm{H} & 1.250998 & -0.909039 & -4.178635 \\ \mathrm{H} & 3.364941 & 0.264505 & -3.624636 \\ \mathrm{H} & 3.354045 & 2.241078 & -2.141733\end{array}$

VII
74

scf done: -3345.249407

$\begin{array}{cccc}\mathrm{C} & 0.270433 & 0.228552 & -0.123238 \\ \mathrm{C} & 0.122625 & 0.105657 & 1.252166 \\ \mathrm{C} & 1.248540 & 0.013302 & 2.085385 \\ \mathrm{C} & 2.518727 & 0.064123 & 1.497314 \\ \mathrm{C} & 2.665751 & 0.191597 & 0.119383 \\ \mathrm{C} & 1.541825 & 0.272610 & -0.696311 \\ \mathrm{C} & 1.154064 & -0.128949 & 3.543360 \\ \mathrm{Pd} & 0.230913 & 1.983683 & 4.260803 \\ \mathrm{~S} & -2.163500 & 2.228279 & 3.862634 \\ \mathrm{C} & -2.929692 & 2.415176 & 5.509671 \\ \mathrm{C} & 0.033290 & -0.407419 & 4.267396 \\ \mathrm{C} & 0.074227 & -0.719547 & 5.727639 \\ \mathrm{O} & -1.144339 & -0.307830 & 6.311323 \\ \mathrm{C} & -1.045004 & -0.062738 & 7.696784 \\ \mathrm{C} & -0.408252 & 1.223752 & 7.985504 \\ \mathrm{C} & 0.092700 & 2.300999 & 8.208886 \\ \mathrm{~S} & 2.536768 & 2.827949 & 4.466333 \\ \mathrm{Pd} & 1.486088 & 4.837032 & 3.646856 \\ \mathrm{O} & 0.881380 & 5.518206 & 5.659343 \\ \mathrm{C} & -0.309256 & 5.521698 & 6.070998 \\ \mathrm{O} & -1.351393 & 5.189451 & 5.462425 \\ \mathrm{Pd} & -1.499938 & 4.455113 & 3.296932 \\ \mathrm{P} & -2.704385 & 4.243012 & 1.374466\end{array}$




$\begin{array}{rrrr}\mathrm{C} & -2.577104 & 5.611623 & 0.176357 \\ \mathrm{C} & 2.783726 & 3.104047 & 6.255582 \\ \mathrm{~S} & -0.112696 & 6.293090 & 2.550946 \\ \mathrm{C} & -0.407085 & 7.721712 & 3.656122 \\ \mathrm{P} & 2.465869 & 4.525626 & 1.598007 \\ \mathrm{C} & 3.925115 & 3.441921 & 1.499434 \\ \mathrm{C} & 3.038363 & 6.082332 & 0.842451 \\ \mathrm{C} & 1.296087 & 3.799664 & 0.404599 \\ \mathrm{C} & -2.417478 & 2.749150 & 0.368404 \\ \mathrm{C} & -4.481519 & 4.180329 & 1.775754 \\ \mathrm{H} & -0.809120 & 8.536370 & 3.045768 \\ \mathrm{H} & 0.540505 & 8.031763 & 4.106567 \\ \mathrm{H} & -1.120279 & 7.457314 & 4.439943 \\ \mathrm{H} & -2.439321 & 3.216856 & 6.068464 \\ \mathrm{H} & -2.813526 & 1.456905 & 6.025921 \\ \mathrm{H} & -3.991935 & 2.644169 & 5.371141 \\ \mathrm{H} & 2.175054 & 3.950159 & 6.586177 \\ \mathrm{H} & 3.844090 & 3.317418 & 6.424755 \\ \mathrm{H} & 2.494874 & 2.197906 & 6.799659 \\ \mathrm{H} & 4.294436 & 3.433565 & 0.464694 \\ \mathrm{H} & 4.712512 & 3.808182 & 2.167076 \\ \mathrm{H} & 3.657952 & 2.419530 & 1.790611 \\ \mathrm{H} & 3.467599 & 5.880218 & -0.148258 \\ \mathrm{H} & 2.203379 & 6.783857 & 0.744417 \\ \mathrm{H} & 3.805559 & 6.536123 & 1.479824 \\ \mathrm{H} & 1.768890 & 3.675537 & -0.579277 \\ \mathrm{H} & 0.973474 & 2.816973 & 0.776701 \\ \mathrm{H} & 0.419937 & 4.454171 & 0.310865 \\ \mathrm{H} & -0.443124 & 5.872305 & 7.115481 \\ \mathrm{H} & -3.280966 & 5.437876 & -0.648662 \\ \mathrm{H} & -2.822953 & 6.559406 & 0.667740 \\ \mathrm{H} & -1.561530 & 5.685551 & -0.227952 \\ \mathrm{H} & -5.073816 & 4.111557 & 0.853038 \\ \mathrm{H} & -4.684939 & 3.307237 & 2.406544 \\ \mathrm{H} & -4.767651 & 5.084216 & 2.325186 \\ \mathrm{H} & -3.090223 & 2.759285 & -0.499756 \\ \mathrm{H} & -1.377932 & 2.715113 & 0.020413 \\ \mathrm{H} & -2.618363 & 1.853428 & 0.967812 \\ \mathrm{H} & 0.529116 & 3.255697 & 8.421572 \\ \mathrm{H} & 0.208621 & -1.805465 & 5.890639 \\ \mathrm{H} & 0.933379 & -0.205561 & 6.196444 \\ \mathrm{H} & -0.506615 & -0.882962 & 8.202964 \\ \mathrm{H} & -2.071262 & -0.066556 & 8.086576 \\ \mathrm{H} & -0.925752 & -0.589943 & 3.778031 \\ \mathrm{H} & 2.110422 & -0.108622 & 4.074728 \\ \mathrm{H} & 3.401442 & -0.014269 & 2.134220 \\ \mathrm{H} & 3.663135 & 0.221726 & -0.316521 \\ \mathrm{H} & 1.652209 & 0.363728 & -1.775165 \\ \mathrm{H} & -0.614814 & 0.282697 & -0.756043 \\ \mathrm{H} & -0.877800 & 0.078953 & 1.685939 \\ \mathrm{H} & -0.003314 & 3.496716 & 4.604929 \\ & & & \end{array}$

\section{TS (VII-VIII)}

74

scf done: -3345.226840
C 0.181526
0.197656
$-0.390845$
C 0.154182
0.214100
0.999442
C 1.339685
0.106123
1.720275 


\begin{tabular}{|c|c|c|c|}
\hline $\mathrm{C}$ & 2.550653 & -0.003695 & 1.037384 \\
\hline $\mathrm{C}$ & 2.579133 & -0.017263 & -0.351737 \\
\hline C & 1.389796 & 0.066587 & -1.089571 \\
\hline $\mathrm{C}$ & 1.323216 & -0.085874 & -2.547717 \\
\hline $\mathrm{C}$ & 2.315287 & -0.526184 & -3.383211 \\
\hline $\mathrm{C}$ & 2.013262 & -1.112618 & -4.735059 \\
\hline $\mathrm{H}$ & 2.839894 & -0.928289 & -5.440605 \\
\hline $\mathrm{Pd}$ & 2.263418 & 1.818639 & -3.624999 \\
\hline $\mathrm{C}$ & 1.707056 & 1.510520 & -5.819435 \\
\hline $\mathrm{C}$ & 2.421048 & 2.525725 & -5.867154 \\
\hline $\mathrm{Pd}$ & 1.002439 & 4.981443 & -2.020580 \\
\hline$P$ & 0.109564 & 4.478259 & 0.017711 \\
\hline$C$ & -1.422988 & 3.499101 & 0.092179 \\
\hline $\mathrm{Pd}$ & 3.923686 & 4.469598 & -1.799513 \\
\hline $\mathrm{S}$ & 2.698820 & 6.367582 & -0.936386 \\
\hline $\mathrm{C}$ & 3.038317 & 7.813982 & -2.008242 \\
\hline $\mathrm{S}$ & 4.474596 & 2.254745 & -2.499638 \\
\hline $\mathrm{C}$ & 5.569211 & 2.578432 & -3.932214 \\
\hline $\mathrm{S}$ & -0.082401 & 3.100583 & -2.972788 \\
\hline $\mathrm{C}$ & -0.697872 & 3.731260 & -4.572508 \\
\hline $\mathrm{O}$ & 1.502728 & 5.788457 & -3.967361 \\
\hline $\mathrm{C}$ & 2.659541 & 5.704597 & -4.439367 \\
\hline $\mathrm{O}$ & 3.671284 & 5.153811 & -3.935678 \\
\hline $\mathrm{P}$ & 5.160155 & 4.125109 & 0.077098 \\
\hline $\mathrm{C}$ & 5.080883 & 5.417549 & 1.362221 \\
\hline $\mathrm{C}$ & 4.959459 & 2.572062 & 1.010398 \\
\hline $\mathrm{C}$ & 6.918725 & 4.115396 & -0.407696 \\
\hline $\mathrm{O}$ & 0.784629 & -0.667917 & -5.262292 \\
\hline $\mathrm{C}$ & 0.892214 & 0.350556 & -6.218867 \\
\hline $\mathrm{C}$ & -0.320351 & 5.983084 & 0.957551 \\
\hline $\mathrm{C}$ & 1.280186 & 3.581286 & 1.088077 \\
\hline $\mathrm{H}$ & 3.476095 & 8.597928 & -1.382640 \\
\hline $\mathrm{H}$ & 2.102800 & 8.175201 & -2.445672 \\
\hline $\mathrm{H}$ & 3.739484 & 7.550583 & -2.803962 \\
\hline $\mathrm{H}$ & 5.221440 & 3.455032 & -4.487651 \\
\hline $\mathrm{H}$ & 5.558613 & 1.695211 & -4.581482 \\
\hline $\mathrm{H}$ & 6.591289 & 2.750543 & -3.576585 \\
\hline $\mathrm{H}$ & 0.118202 & 4.105904 & -5.196501 \\
\hline $\mathrm{H}$ & -1.423824 & 4.533105 & -4.405242 \\
\hline $\mathrm{H}$ & -1.201382 & 2.900205 & -5.081252 \\
\hline $\mathrm{H}$ & -1.720040 & 3.378241 & 1.143043 \\
\hline $\mathrm{H}$ & -2.218422 & 4.014007 & -0.457873 \\
\hline $\mathrm{H}$ & -1.268368 & 2.516152 & -0.362494 \\
\hline $\mathrm{H}$ & -0.753820 & 5.706214 & 1.928257 \\
\hline $\mathrm{H}$ & 0.573538 & 6.595929 & 1.115158 \\
\hline $\mathrm{H}$ & -1.052116 & 6.571898 & 0.392935 \\
\hline $\mathrm{H}$ & 0.812203 & 3.328539 & 2.049291 \\
\hline $\mathrm{H}$ & 1.599041 & 2.657221 & 0.587212 \\
\hline $\mathrm{H}$ & 2.154713 & 4.218804 & 1.268745 \\
\hline $\mathrm{H}$ & 2.802933 & 6.165612 & -5.438520 \\
\hline $\mathrm{H}$ & 5.788255 & 5.170187 & 2.165120 \\
\hline $\mathrm{H}$ & 5.346929 & 6.387837 & 0.928351 \\
\hline $\mathrm{H}$ & 4.073111 & 5.496204 & 1.784536 \\
\hline $\mathrm{H}$ & 7.555943 & 4.074135 & 0.486178 \\
\hline $\mathrm{H}$ & 7.121082 & 3.239777 & -1.035137 \\
\hline $\mathrm{H}$ & 7.154732 & 5.020770 & -0.978017 \\
\hline $\mathrm{H}$ & 5.713785 & 2.531376 & 1.807951 \\
\hline $\mathrm{H}$ & 3.959668 & 2.513904 & 1.455909 \\
\hline $\mathrm{H}$ & 5.095742 & 1.718535 & 0.336785 \\
\hline $\mathrm{H}$ & 3.041186 & 3.300900 & -6.285014 \\
\hline
\end{tabular}




$\begin{array}{rrrr}\mathrm{H} & 1.930980 & -2.204859 & -4.623506 \\ \mathrm{H} & 3.313997 & -0.743030 & -2.997928 \\ \mathrm{H} & 0.316109 & -0.047340 & -2.968608 \\ \mathrm{H} & -0.749206 & 0.253170 & -0.958128 \\ \mathrm{H} & -0.799002 & 0.302443 & 1.519347 \\ \mathrm{H} & 1.322210 & 0.103235 & 2.808530 \\ \mathrm{H} & 3.482785 & -0.093809 & 1.594084 \\ \mathrm{H} & 3.534483 & -0.116906 & -0.867456 \\ \mathrm{H} & 2.651501 & 3.214953 & -4.245786 \\ \mathrm{H} & -0.130132 & 0.682888 & -6.438346 \\ \mathrm{H} & 1.317701 & -0.046514 & -7.158692\end{array}$

\section{VIII}

74

scf done: -3345.290961

$\begin{array}{llll}\text { C } & -2.197406 & -0.181635 & 1.032593\end{array}$

$\begin{array}{llll}\text { C } & -1.198011 & 0.100779 & 1.952002\end{array}$

$\begin{array}{llll}\text { C } & 0.136471 & 0.222158 & 1.536677\end{array}$

$\begin{array}{llll}\text { C } & 0.438314 & 0.058265 & 0.179975\end{array}$

$\begin{array}{llll}\text { C } & -0.564196 & -0.231171 & -0.739927\end{array}$

$\begin{array}{llll}\text { C } & -1.883902 & -0.349853 & -0.316680\end{array}$

$\begin{array}{llll}\text { C } & 1.224516 & 0.516700 & 2.475900\end{array}$

$\begin{array}{llll}\text { C } & 1.174134 & 0.279596 & 3.829632\end{array}$

$\begin{array}{llll}\text { C } & 2.376328 & 0.180787 & 4.722796\end{array}$

$\begin{array}{llll}\text { O } & 2.065158 & 0.441234 & 6.066918\end{array}$

$\begin{array}{llll}\text { C } & 1.552411 & 1.738200 & 6.297244\end{array}$

$\begin{array}{llll}\text { C } & 0.348954 & 2.029021 & 5.453996\end{array}$

$\begin{array}{llll}\mathrm{Pd} & 0.835749 & 2.594725 & 3.534476\end{array}$

$\begin{array}{llll}\text { S } & 1.367140 & 3.276362 & 1.077005\end{array}$

$\begin{array}{llll}\text { C } & -0.354688 & 3.477038 & 0.497704\end{array}$

$\begin{array}{llll}\text { C } & -0.910019 & 1.896759 & 5.860617\end{array}$

$\begin{array}{llll}\mathrm{S} & 0.615183 & 4.727347 & 4.769317\end{array}$

$\begin{array}{llll}\text { C } & -1.166591 & 5.109175 & 4.789143\end{array}$

$\begin{array}{llll}\mathrm{Pd} & 1.530861 & 6.544777 & 3.530653\end{array}$

$\begin{array}{llll}\mathrm{P} & 3.004582 & 6.852781 & 5.240932\end{array}$

$\begin{array}{llll}\mathrm{C} & 4.019037 & 5.414884 & 5.723207\end{array}$

$\begin{array}{llll}\mathrm{O} & -0.306209 & 6.744414 & 2.336361\end{array}$

$\begin{array}{llll}\text { C } & -0.405250 & 6.694019 & 1.088942\end{array}$

$\begin{array}{llll}\mathrm{O} & 0.449972 & 6.296306 & 0.257534\end{array}$

$\begin{array}{llll}\mathrm{Pd} & 2.265963 & 5.478558 & 1.012948\end{array}$

$\begin{array}{llll}\text { S } & 2.913149 & 7.682859 & 1.869055\end{array}$

$\begin{array}{llll}\text { C } & 1.882582 & 8.945810 & 1.034320\end{array}$

$\begin{array}{llll}\mathrm{P} & 4.313770 & 4.551540 & 1.415217\end{array}$

$\begin{array}{llll}\text { C } & 4.975299 & 3.830558 & -0.122128\end{array}$

$\begin{array}{llll}\text { C } & 5.635410 & 5.679783 & 1.969745\end{array}$

$\begin{array}{llll}\text { C } & 4.362594 & 3.164320 & 2.603704\end{array}$

$\begin{array}{llll}\text { C } & 4.204650 & 8.209013 & 5.030380\end{array}$

$\begin{array}{llll}\text { C } & 2.112400 & 7.301085 & 6.765913\end{array}$

$\begin{array}{llll}\mathrm{H} & -1.766679 & 2.026782 & 5.197197\end{array}$

$\begin{array}{llll}\mathrm{H} & -0.787989 & 2.472708 & 0.425854\end{array}$

$\begin{array}{llll}\mathrm{H} & -0.364723 & 3.953353 & -0.486122\end{array}$

$\begin{array}{llll}\mathrm{H} & -0.936649 & 4.076250 & 1.206508\end{array}$

$\begin{array}{llll}\mathrm{H} & -1.557307 & 5.198284 & 3.773039\end{array}$

$\begin{array}{llll}\mathrm{H} & -1.305844 & 6.059065 & 5.316965\end{array}$

$\begin{array}{llll}\mathrm{H} & -1.673262 & 4.308073 & 5.337954\end{array}$

$\begin{array}{llll}\mathrm{H} & 1.506966 & 8.552480 & 0.086173\end{array}$

$\begin{array}{llll}\mathrm{H} & 2.511891 & 9.821853 & 0.851291\end{array}$

$\begin{array}{llll}\mathrm{H} & 1.039327 & 9.226396 & 1.672453\end{array}$ 


$\begin{array}{rrrr}\mathrm{H} & 6.581632 & 5.128960 & 2.052343 \\ \mathrm{H} & 5.750248 & 6.495896 & 1.247819 \\ \mathrm{H} & 5.389902 & 6.121153 & 2.942703 \\ \mathrm{H} & 5.945848 & 3.352547 & 0.066388 \\ \mathrm{H} & 4.267434 & 3.087267 & -0.506402 \\ \mathrm{H} & 5.098692 & 4.616253 & -0.876038 \\ \mathrm{H} & 5.402833 & 2.910099 & 2.847969 \\ \mathrm{H} & 3.809937 & 3.412673 & 3.520013 \\ \mathrm{H} & 3.881690 & 2.292563 & 2.142737 \\ \mathrm{H} & 4.818899 & 8.299389 & 5.936050 \\ \mathrm{H} & 4.850743 & 8.033535 & 4.163538 \\ \mathrm{H} & 3.664677 & 9.147703 & 4.862449 \\ \mathrm{H} & 4.698480 & 5.124684 & 4.911896 \\ \mathrm{H} & 4.615655 & 5.661481 & 6.611535 \\ \mathrm{H} & 3.363063 & 4.568104 & 5.960066 \\ \mathrm{H} & 1.515086 & 8.202920 & 6.590020 \\ \mathrm{H} & 1.443376 & 6.483386 & 7.056022 \\ \mathrm{H} & 2.828990 & 7.493644 & 7.576086 \\ \mathrm{H} & -1.363717 & 7.042000 & 0.659169 \\ \mathrm{H} & -1.136983 & 1.640910 & 6.900137 \\ \mathrm{H} & 2.344696 & 2.493374 & 6.123758 \\ \mathrm{H} & 1.287124 & 1.763074 & 7.363991 \\ \mathrm{H} & 2.750773 & -0.852544 & 4.707760 \\ \mathrm{H} & 3.191563 & 0.834389 & 4.357003 \\ \mathrm{H} & 0.269217 & -0.159071 & 4.249794 \\ \mathrm{H} & 2.199421 & 0.709426 & 2.019886 \\ \mathrm{H} & 1.471473 & 0.162338 & -0.151352 \\ \mathrm{H} & -0.313258 & -0.360065 & -1.790862 \\ \mathrm{H} & -2.670614 & -0.569516 & -1.035598 \\ \mathrm{H} & -3.229325 & -0.268431 & 1.367168 \\ \mathrm{H} & -1.456840 & 0.247727 & 3.001409\end{array}$

\section{TS (VIII-IX) \\ 74}

scf done: -3345.284389

$\begin{array}{cccc}\mathrm{C} & 0.074977 & -0.075429 & 0.060563 \\ \mathrm{C} & 0.025924 & -0.047294 & 1.454118 \\ \mathrm{C} & 1.198685 & -0.006382 & 2.193949 \\ \mathrm{C} & 2.448075 & 0.010369 & 1.554113 \\ \mathrm{C} & 2.483370 & -0.017380 & 0.153563 \\ \mathrm{C} & 1.307159 & -0.063147 & -0.586611 \\ \mathrm{C} & 3.705515 & 0.070936 & 2.306449 \\ \mathrm{Pd} & 3.940124 & 1.966286 & 3.351751 \\ \mathrm{~S} & 4.346078 & 3.881233 & 4.958914 \\ \mathrm{Pd} & 5.328923 & 5.721103 & 3.817608 \\ \mathrm{~S} & 6.525419 & 6.846745 & 2.000271 \\ \mathrm{C} & 5.600777 & 8.361784 & 1.548172 \\ \mathrm{C} & 3.830534 & -0.392814 & 3.640799 \\ \mathrm{C} & 5.159088 & -0.906210 & 4.134854 \\ \mathrm{O} & 5.259458 & -0.791698 & 5.528346 \\ \mathrm{C} & 4.938548 & 0.529131 & 5.911497 \\ \mathrm{C} & 3.683217 & 0.950977 & 5.212967 \\ \mathrm{C} & 2.495474 & 1.083690 & 5.807836 \\ \mathrm{~S} & 4.063367 & 2.906175 & 1.018385 \\ \mathrm{Pd} & 5.307905 & 4.932497 & 1.081944 \\ \mathrm{P} & 7.200930 & 3.707091 & 0.716725 \\ \mathrm{C} & 7.294097 & 2.055640 & 1.492397 \\ \mathrm{C} & 2.322158 & 3.444397 & 0.880957\end{array}$




$\begin{array}{rrrr}\mathrm{C} & 2.675029 & 4.469627 & 5.391454 \\ \mathrm{P} & 7.164670 & 5.421551 & 5.127450 \\ \mathrm{C} & 6.745321 & 5.733325 & 6.874182 \\ \mathrm{O} & 3.365804 & 6.446139 & 3.164432 \\ \mathrm{C} & 2.982369 & 6.556780 & 1.977560 \\ \mathrm{O} & 3.537008 & 6.123261 & 0.936630 \\ \mathrm{C} & 7.913793 & 3.757676 & 5.135526 \\ \mathrm{C} & 8.569807 & 6.541425 & 4.816690 \\ \mathrm{C} & 7.353395 & 3.363723 & -1.067165 \\ \mathrm{C} & 8.784811 & 4.493624 & 1.162367 \\ \mathrm{H} & 1.577792 & 1.287715 & 5.253644 \\ \mathrm{H} & 1.714302 & 2.536082 & 0.792468 \\ \mathrm{H} & 2.195053 & 4.063974 & -0.010790 \\ \mathrm{H} & 2.017150 & 4.006023 & 1.770131 \\ \mathrm{H} & 2.142943 & 4.825775 & 4.506290 \\ \mathrm{H} & 2.761412 & 5.283803 & 6.118455 \\ \mathrm{H} & 2.138221 & 3.631224 & 5.850239 \\ \mathrm{H} & 4.928538 & 8.155226 & 0.711111 \\ \mathrm{H} & 6.330715 & 9.124033 & 1.259702 \\ \mathrm{H} & 5.019989 & 8.716692 & 2.404576 \\ \mathrm{H} & 9.619763 & 3.832912 & 0.894980 \\ \mathrm{H} & 8.888323 & 5.447927 & 0.634428 \\ \mathrm{H} & 8.818542 & 4.698088 & 2.239532 \\ \mathrm{H} & 8.262694 & 2.782749 & -1.272872 \\ \mathrm{H} & 6.474605 & 2.800346 & -1.402038 \\ \mathrm{H} & 7.393738 & 4.307597 & -1.622484 \\ \mathrm{H} & 8.253532 & 1.581613 & 1.246467 \\ \mathrm{H} & 7.192026 & 2.129871 & 2.583201 \\ \mathrm{H} & 6.479200 & 1.428305 & 1.110466 \\ \mathrm{H} & 9.353664 & 6.359861 & 5.564258 \\ \mathrm{H} & 8.981308 & 6.387790 & 3.813102 \\ \mathrm{H} & 8.233905 & 7.581929 & 4.889473 \\ \mathrm{H} & 8.382265 & 3.541363 & 4.167594 \\ \mathrm{H} & 8.682804 & 3.697714 & 5.916997 \\ \mathrm{H} & 7.141510 & 3.004414 & 5.334024 \\ \mathrm{H} & 6.327404 & 6.740955 & 6.979627 \\ \mathrm{H} & 5.997637 & 5.005217 & 7.207484 \\ \mathrm{H} & 7.645724 & 5.648433 & 7.497566 \\ \mathrm{H} & 2.034975 & 7.106325 & 1.819330 \\ \mathrm{H} & 2.397881 & 1.002469 & 6.893484 \\ \mathrm{H} & 5.774172 & 1.210572 & 5.659113 \\ \mathrm{H} & 4.810149 & 0.525883 & 7.001895 \\ \mathrm{H} & 5.257694 & -1.973228 & 3.897289 \\ \mathrm{H} & 5.978584 & -0.360227 & 3.626571 \\ \mathrm{H} & 2.964610 & -0.908259 & 4.054256 \\ \mathrm{H} & 4.616729 & 0.073045 & 1.698638 \\ \mathrm{H} & 3.447750 & -0.002861 & -0.354191 \\ \mathrm{H} & 1.354496 & -0.089152 & -1.673427 \\ \mathrm{H} & -0.846159 & -0.108989 & -0.517605 \\ \mathrm{H} & -0.934539 & -0.058913 & 1.965777 \\ \mathrm{H} & 1.143251 & 0.022021 & 3.282890 \\ & & & \end{array}$

\section{IX}

74

scf done: -3345.326985
C $\quad-0.088444 \quad-0.133061$
0.388587
$\begin{array}{llll}\text { C } & -0.048350 & -0.275557 & 1.773948\end{array}$
$\begin{array}{llll}\text { C } & 1.159630 & -0.182195 & 2.453061\end{array}$ 


\begin{tabular}{|c|c|c|c|}
\hline $\mathrm{C}$ & 2.358146 & 0.067120 & 1.765081 \\
\hline $\mathrm{C}$ & 2.300655 & 0.190115 & 0.366084 \\
\hline $\mathrm{C}$ & 1.093491 & 0.089457 & -0.313651 \\
\hline $\mathrm{C}$ & 3.652951 & 0.196756 & 2.448058 \\
\hline $\mathrm{Pd}$ & 3.864366 & 2.129451 & 3.364796 \\
\hline $\mathrm{S}$ & 4.140358 & 4.134741 & 5.002896 \\
\hline $\mathrm{Pd}$ & 5.266706 & 5.868340 & 3.834013 \\
\hline S & 6.589969 & 6.861649 & 2.030430 \\
\hline $\mathrm{C}$ & 5.745024 & 8.387286 & 1.469103 \\
\hline $\mathrm{C}$ & 3.941992 & -0.635518 & 3.679851 \\
\hline $\mathrm{C}$ & 5.359693 & -1.227653 & 3.718128 \\
\hline $\mathrm{O}$ & 5.781057 & -1.122876 & 5.061483 \\
\hline $\mathrm{C}$ & 5.363671 & 0.14 & 5.498384 \\
\hline $\mathrm{C}$ & 4.018370 & 0.341412 & 4.842763 \\
\hline $\mathrm{C}$ & 3.016107 & 1.159442 & 5.272072 \\
\hline S & 4.020989 & 2.982664 & 1.068382 \\
\hline $\mathrm{Pd}$ & 5.338836 & 4.964594 & 1.129519 \\
\hline $\mathrm{P}$ & 7.192926 & 3.65 & 0.859217 \\
\hline $\mathrm{C}$ & 7.166479 & 1.996212 & 1.629742 \\
\hline $\mathrm{C}$ & 2.295 & 3.572356 & 0.913475 \\
\hline $\mathrm{C}$ & 2.417 & 4.72 & 5.17 \\
\hline $\mathrm{P}$ & 7.037061 & 5.519961 & 5.221661 \\
\hline $\mathrm{C}$ & 6.59 & 6.01 & 6.919900 \\
\hline $\mathrm{O}$ & 3.38 & 6.6 & 3.061826 \\
\hline $\mathrm{C}$ & 3.046 & 6.718789 & 1.857434 \\
\hline $\mathrm{O}$ & 3.6 & 15 & 0.8 \\
\hline $\mathrm{C}$ & 7.629436 & 3.801984 & 5.386554 \\
\hline $\mathrm{C}$ & 8.54 & 6.48 & 4.86 \\
\hline $\mathrm{C}$ & 7.40 & 3.3 & -0.917574 \\
\hline $\mathrm{C}$ & 8.796910 & 4.348902 & 1.376478 \\
\hline $\mathrm{H}$ & 1.99 & 1.0 & 4.88 \\
\hline $\mathrm{H}$ & 1.65 & 2.6 & 0.879431 \\
\hline $\mathrm{H}$ & 2.190195 & 4.145082 & -0.012248 \\
\hline $\mathrm{H}$ & 2.0 & 4.1 & 1.766576 \\
\hline $\mathrm{H}$ & 1.979891 & 4.947251 & 4.200372 \\
\hline $\mathrm{H}$ & 2.398101 & 5.6 & 5.794400 \\
\hline $\mathrm{H}$ & 1.84 & 3.9 & 5.67 \\
\hline $\mathrm{H}$ & 5.115496 & 8.169586 & 0.602031 \\
\hline $\mathrm{H}$ & 6.51 & 9.11 & 1.196744 \\
\hline $\mathrm{H}$ & 5.127679 & 8.792046 & 2.276262 \\
\hline $\mathrm{H}$ & 9.610199 & 3.686671 & 1.052617 \\
\hline $\mathrm{H}$ & 8.93 & & 0.936267 \\
\hline $\mathrm{H}$ & 8.834613 & 4.445776 & 2.468184 \\
\hline $\mathrm{H}$ & 8.268447 & 2.641508 & -1.076761 \\
\hline $\mathrm{H}$ & 6.500 & 2.829151 & -1.306525 \\
\hline $\mathrm{H}$ & 7.569250 & 4.244632 & -1.459356 \\
\hline $\mathrm{H}$ & 8.145063 & 1.511857 & 1.512082 \\
\hline $\mathrm{H}$ & 6.912271 & 2.068677 & 2.695649 \\
\hline $\mathrm{H}$ & 6.403526 & 1.379206 & 1.138831 \\
\hline $\mathrm{H}$ & 9.308692 & 6.260103 & 5.622122 \\
\hline $\mathrm{H}$ & 8.931632 & 6.262990 & 3.871017 \\
\hline $\mathrm{H}$ & 8.302319 & 7.555752 & 4.906453 \\
\hline $\mathrm{H}$ & 8.092355 & 3.465255 & 4.450784 \\
\hline $\mathrm{H}$ & 8.375238 & 3.737631 & 6.190189 \\
\hline $\mathrm{H}$ & 6.786676 & 3.141383 & 5.627016 \\
\hline $\mathrm{H}$ & 6.250553 & 7.051912 & 6.922868 \\
\hline $\mathrm{H}$ & 5.785913 & 5.371291 & 7.285054 \\
\hline $\mathrm{H}$ & 7.468723 & 5.919535 & 7.580152 \\
\hline $\mathrm{H}$ & 2.133253 & 7.303290 & 1.636638 \\
\hline $\mathrm{H}$ & 3.149330 & 1.757209 & 6.173437 \\
\hline
\end{tabular}




$\begin{array}{rrrr}\mathrm{H} & 6.079407 & 0.933064 & 5.181027 \\ \mathrm{H} & 5.312143 & 0.153086 & 6.593436 \\ \mathrm{H} & 5.410905 & -2.278990 & 3.421476 \\ \mathrm{H} & 6.029207 & -0.638179 & 3.060721 \\ \mathrm{H} & 3.192879 & -1.421791 & 3.860029 \\ \mathrm{H} & 4.489373 & 0.197623 & 1.739414 \\ \mathrm{H} & 3.223419 & 0.368188 & -0.186013 \\ \mathrm{H} & 1.075268 & 0.182370 & -1.397963 \\ \mathrm{H} & -1.035718 & -0.209194 & -0.141423 \\ \mathrm{H} & -0.964986 & -0.467839 & 2.328643 \\ \mathrm{H} & 1.170636 & -0.311741 & 3.535463\end{array}$

$\mathrm{X}$

74

scf done: -3345.330020

$\begin{array}{llll}\text { C } & 1.394527 & -0.919900 & 1.619120 \\ \text { C } & 0.514432 & -0.641458 & 2.681439\end{array}$

$\begin{array}{llll}\text { C } & 0.514432 & -0.641458 & 2.681439\end{array}$

$\begin{array}{llll}\text { C } & 1.052564 & -0.531769 & 3.975266\end{array}$

$\begin{array}{llll}\text { C } & 2.411956 & -0.703932 & 4.195101\end{array}$

$\begin{array}{llll}\text { C } & 3.265198 & -0.999659 & 3.133163\end{array}$

$\begin{array}{llll}\text { C } & 2.751067 & -1.105329 & 1.842084\end{array}$

$\begin{array}{llll}\text { C } & -0.919929 & -0.486923 & 2.391820\end{array}$

$\begin{array}{llll}\mathrm{Pd} & -1.444157 & -2.498872 & 2.251803\end{array}$

$\begin{array}{llll}\mathrm{S} & -1.067093 & -3.359881 & 4.541614\end{array}$

$\begin{array}{llll}\mathrm{Pd} & -2.427604 & -5.056704 & 3.558377\end{array}$

$\begin{array}{llll}\mathrm{P} & -4.214014 & -4.371379 & 4.826110\end{array}$

$\begin{array}{llll}\text { C } & -5.773111 & -4.083486 & 3.924289\end{array}$

$\begin{array}{llll}\text { C } & -1.809440 & 0.205213 & 3.400706\end{array}$

$\begin{array}{llll}\text { C } & -3.251939 & 0.346192 & 2.946589\end{array}$

$\begin{array}{llll}\mathrm{C} & -3.485585 & 1.815873 & 2.700772\end{array}$

$\begin{array}{llll}\mathrm{O} & -2.195681 & 2.376844 & 2.584049\end{array}$

$\begin{array}{llll}\text { C } & -1.411701 & 1.698227 & 3.536149\end{array}$

$\begin{array}{llll}\text { C } & -4.153510 & -0.619057 & 2.788454\end{array}$

$\begin{array}{llll}\mathrm{S} & -2.320355 & -2.302555 & 0.003198\end{array}$

$\begin{array}{llll}\mathrm{Pd} & -3.094865 & -4.473153 & 0.647011\end{array}$

$\begin{array}{llll}\mathrm{S} & -3.899031 & -6.295153 & 2.048379\end{array}$

$\begin{array}{llll}\text { C } & -2.961102 & -7.797933 & 1.574199\end{array}$

$\begin{array}{llll}\text { C } & -0.938528 & -2.651603 & -1.139773\end{array}$

$\begin{array}{llll}\text { C } & 0.652058 & -3.991719 & 4.472968\end{array}$

$\begin{array}{llll}\mathrm{O} & -0.693884 & -5.995024 & 2.734184\end{array}$

$\begin{array}{llll}\text { C } & -0.376785 & -5.815588 & 1.533325\end{array}$

$\begin{array}{llll}\mathrm{O} & -0.993995 & -5.174886 & 0.650874\end{array}$

$\begin{array}{llll}\mathrm{P} & -5.112158 & -3.955177 & -0.287777\end{array}$

$\begin{array}{llll}\text { C } & -4.873432 & -3.834794 & -2.091629\end{array}$

$\begin{array}{llll}\text { C } & -6.434693 & -5.195574 & -0.105213\end{array}$

$\begin{array}{llll}\text { C } & -5.908432 & -2.381399 & 0.169867\end{array}$

$\begin{array}{llll}\text { C } & -4.614472 & -5.708904 & 5.997596\end{array}$

$\begin{array}{llll}\text { C } & -4.059251 & -2.882684 & 5.867069\end{array}$

$\begin{array}{llll}\mathrm{H} & -3.896551 & -1.666808 & 2.963296\end{array}$

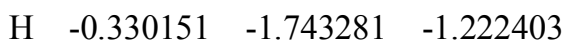

$\begin{array}{llll}\mathrm{H} & -1.345880 & -2.892557 & -2.128276\end{array}$

$\begin{array}{llll}\mathrm{H} & -0.335012 & -3.484341 & -0.769928\end{array}$

$\begin{array}{llll}\mathrm{H} & 1.082069 & -3.875125 & 3.473571\end{array}$

$\begin{array}{llll}\mathrm{H} & 0.672992 & -5.046014 & 4.761466\end{array}$

$\begin{array}{llll}\mathrm{H} & 1.236018 & -3.403161 & 5.188709\end{array}$

$\begin{array}{llll}\mathrm{H} & -2.535014 & -7.678493 & 0.573721\end{array}$

$\begin{array}{llll}\mathrm{H} & -3.657875 & -8.641574 & 1.576889\end{array}$

$\begin{array}{llll}\mathrm{H} & -2.156473 & -7.976907 & 2.292402\end{array}$ 


$\begin{array}{rrrr}\text { H } & -7.312542 & -4.878107 & -0.683878 \\ \mathrm{H} & -6.087147 & -6.165444 & -0.477829 \\ \mathrm{H} & -6.717680 & -5.309841 & 0.946318 \\ \mathrm{H} & -5.840479 & -3.681590 & -2.589965 \\ \mathrm{H} & -4.209762 & -2.992754 & -2.318033 \\ \mathrm{H} & -4.416608 & -4.757221 & -2.467881 \\ \mathrm{H} & -6.758261 & -2.198148 & -0.501425 \\ \mathrm{H} & -6.278835 & -2.426860 & 1.200511 \\ \mathrm{H} & -5.191163 & -1.557393 & 0.081702 \\ \mathrm{H} & -5.496000 & -5.438602 & 6.594552 \\ \mathrm{H} & -4.819276 & -6.632282 & 5.443285 \\ \mathrm{H} & -3.763573 & -5.881789 & 6.666048 \\ \mathrm{H} & -6.045158 & -4.981422 & 3.357350 \\ \mathrm{H} & -6.574757 & -3.845823 & 4.635717 \\ \mathrm{H} & -5.645571 & -3.242888 & 3.231914 \\ \mathrm{H} & -3.195023 & -2.968048 & 6.533819 \\ \mathrm{H} & -3.938375 & -1.987197 & 5.243361 \\ \mathrm{H} & -4.973776 & -2.776720 & 6.465724 \\ \mathrm{H} & 0.573195 & -6.287342 & 1.217103 \\ \mathrm{H} & -5.170292 & -0.398366 & 2.460914 \\ \mathrm{H} & -4.049803 & 2.029204 & 1.785810 \\ \mathrm{H} & -4.025440 & 2.271672 & 3.553473 \\ \mathrm{H} & -1.645542 & 2.051011 & 4.558248 \\ \mathrm{H} & -0.356683 & 1.900924 & 3.324640 \\ \mathrm{H} & -1.754533 & -0.307169 & 4.372128 \\ \mathrm{H} & -1.101590 & -0.109713 & 1.375978 \\ \mathrm{H} & 0.990505 & -0.989740 & 0.608283 \\ \mathrm{H} & 3.413166 & -1.320816 & 1.005663 \\ \mathrm{H} & 4.330375 & -1.135183 & 3.309746 \\ \mathrm{H} & 2.812210 & -0.603292 & 5.202451 \\ \mathrm{H} & 0.400825 & -0.305436 & 4.818636\end{array}$

\section{TS (X-XI)}

74

scf done: -3345.290680

$\begin{array}{cccc}\mathrm{C} & -0.018623 & 0.061655 & 0.009252 \\ \mathrm{C} & -0.024676 & 0.017008 & 1.412376 \\ \mathrm{C} & 1.208410 & 0.021678 & 2.081160 \\ \mathrm{C} & 2.404389 & 0.095494 & 1.378155 \\ \mathrm{C} & 2.393755 & 0.152809 & -0.012156 \\ \mathrm{C} & 1.177783 & 0.129709 & -0.692829 \\ \mathrm{C} & -1.243224 & -0.069918 & 2.228942 \\ \mathrm{Pd} & -2.471912 & -1.792608 & 1.569229 \\ \mathrm{~S} & -0.543236 & -3.189137 & 2.451894 \\ \mathrm{C} & 0.453807 & -3.525755 & 0.960832 \\ \mathrm{C} & -2.498976 & 0.497813 & 1.950853 \\ \mathrm{C} & -2.858601 & 1.444201 & 0.820180 \\ \mathrm{O} & -4.249417 & 1.622123 & 0.934127 \\ \mathrm{C} & -4.554739 & 1.662769 & 2.316465 \\ \mathrm{C} & -3.500700 & 0.825292 & 3.002915 \\ \mathrm{C} & -3.467439 & 0.524752 & 4.300285 \\ \mathrm{~S} & -4.159881 & -3.175028 & 0.410686 \\ \mathrm{C} & -3.222595 & -3.583185 & -1.102702 \\ \mathrm{Pd} & -4.143719 & -5.145223 & 1.728153 \\ \mathrm{P} & -5.735690 & -4.180776 & 3.068468 \\ \mathrm{C} & -5.776046 & -2.359772 & 3.199496 \\ \mathrm{Pd} & -1.581103 & -5.256206 & 3.020342 \\ \mathrm{P} & -1.446870 & -4.604971 & 5.199374\end{array}$




$\begin{array}{rrrr}\mathrm{C} & 0.293852 & -4.551002 & 5.734187 \\ \mathrm{~S} & -3.450051 & -6.785644 & 3.398981 \\ \mathrm{C} & -3.102458 & -8.349160 & 2.511276 \\ \mathrm{O} & -1.091534 & -6.113354 & 1.091758 \\ \mathrm{C} & -1.907049 & -6.435253 & 0.196959 \\ \mathrm{O} & -3.145213 & -6.229406 & 0.159780 \\ \mathrm{C} & -7.363737 & -4.612598 & 2.371358 \\ \mathrm{C} & -5.838716 & -4.725676 & 4.806773 \\ \mathrm{C} & -2.079050 & -2.932810 & 5.566579 \\ \mathrm{C} & -2.245180 & -5.681805 & 6.434742 \\ \mathrm{H} & -3.583814 & -0.729403 & 1.140914 \\ \mathrm{H} & -1.483728 & -6.971282 & -0.673524 \\ \mathrm{H} & -3.206584 & -2.678139 & -1.720660 \\ \mathrm{H} & -3.729846 & -4.389365 & -1.639655 \\ \mathrm{H} & -2.197003 & -3.885527 & -0.868656 \\ \mathrm{H} & -0.127914 & -4.068056 & 0.211582 \\ \mathrm{H} & 1.328930 & -4.122079 & 1.237053 \\ \mathrm{H} & 0.779735 & -2.557865 & 0.561152 \\ \mathrm{H} & -3.448272 & -8.282332 & 1.476749 \\ \mathrm{H} & -3.634718 & -9.153236 & 3.028628 \\ \mathrm{H} & -2.027916 & -8.553586 & 2.527731 \\ \mathrm{H} & -6.736814 & -4.295601 & 5.269450 \\ \mathrm{H} & -5.889170 & -5.818413 & 4.860737 \\ \mathrm{H} & -4.958900 & -4.385889 & 5.367387 \\ \mathrm{H} & -8.168911 & -4.189318 & 2.987039 \\ \mathrm{H} & -7.437315 & -4.216961 & 1.351547 \\ \mathrm{H} & -7.471175 & -5.702355 & 2.332515 \\ \mathrm{H} & -6.591117 & -2.062390 & 3.872943 \\ \mathrm{H} & -4.822395 & -1.975100 & 3.584618 \\ \mathrm{H} & -5.948109 & -1.911552 & 2.213517 \\ \mathrm{H} & -2.066245 & -5.281282 & 7.441478 \\ \mathrm{H} & -3.322929 & -5.756557 & 6.255745 \\ \mathrm{H} & -1.821647 & -6.690050 & 6.362882 \\ \mathrm{H} & -3.149635 & -2.867045 & 5.334910 \\ \mathrm{H} & -1.923032 & -2.690345 & 6.626229 \\ \mathrm{H} & -1.541049 & -2.204768 & 4.946215 \\ \mathrm{H} & 0.743304 & -5.543640 & 5.618734 \\ \mathrm{H} & 0.844788 & -3.840928 & 5.107816 \\ \mathrm{H} & 0.356736 & -4.245101 & 6.787103 \\ \mathrm{H} & -1.047706 & -0.281610 & 3.284326 \\ \mathrm{H} & -2.660650 & -0.056735 & 4.745143 \\ \mathrm{H} & -4.249246 & 0.866357 & 4.976563 \\ \mathrm{H} & -5.573355 & 1.280419 & 2.456057 \\ \mathrm{H} & -4.524164 & 2.702055 & 2.688098 \\ \mathrm{H} & -2.316833 & 2.396618 & 0.988110 \\ \mathrm{H} & -2.641914 & 1.094910 & -0.190700 \\ \mathrm{H} & 1.221396 & -0.028529 & 3.170092 \\ \mathrm{H} & 3.348424 & 0.105210 & 1.919294 \\ \mathrm{H} & 3.328884 & 0.207914 & -0.565601 \\ \mathrm{H} & 1.160533 & 0.159430 & -1.780455 \\ \mathrm{H} & -0.953430 & 0.002848 & -0.545803\end{array}$

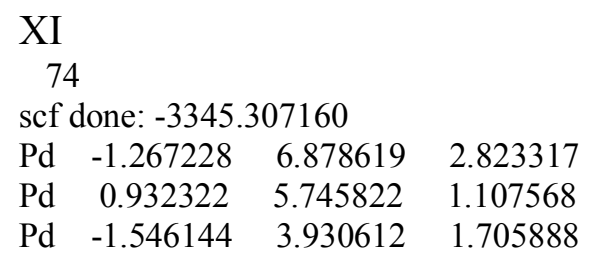




\begin{tabular}{|c|c|c|c|}
\hline H & -0.486750 & 860078 & .465004 \\
\hline$s$ & 0.897969 & 7.859331 & 2.318651 \\
\hline & 0.488558 & 3.507468 & 0.364203 \\
\hline & -2.990730 & 5.250505 & 3.220444 \\
\hline & -0.981972 & 6.993337 & 5.088820 \\
\hline & 2.636580 & 4.840995 & 2.351485 \\
\hline 0 & -0.303439 & 6.590767 & -0.475813 \\
\hline $\mathrm{C}$ & -1.443824 & 7.080405 & -0.283712 \\
\hline $\mathrm{O}$ & -2.045976 & 7.274841 & 0.797920 \\
\hline $\mathrm{C}$ & 0.041736 & 3.702470 & -1.394665 \\
\hline $\mathrm{C}$ & -4.397558 & 5.897165 & 2.251353 \\
\hline $\mathrm{C}$ & -3.417565 & 2.750929 & 0.554188 \\
\hline $\mathrm{C}$ & -3.338314 & 3.036106 & -0.882115 \\
\hline $\mathrm{C}$ & -3.571320 & 4.344211 & -1.333573 \\
\hline $\mathrm{C}$ & -2.755561 & 1.774693 & 1.239328 \\
\hline $\mathrm{C}$ & -1.701090 & 0.817051 & 0.742085 \\
\hline $\mathrm{O}$ & -0.950035 & 0.494995 & 1.892864 \\
\hline $\mathrm{C}$ & -1.866085 & 057 & 2.96 \\
\hline $\mathrm{C}$ & -2.974016 & 1.381960 & 2.642615 \\
\hline $\mathrm{C}$ & -3.972638 & 1.738220 & 3.45 \\
\hline $\mathrm{C}$ & -3.5 & 4.6 & -2.6 \\
\hline $\mathrm{C}$ & -3.220118 & 3.652542 & -3.615807 \\
\hline $\mathrm{C}$ & -3.0 & 2.34 & -3.18 \\
\hline $\mathrm{C}$ & -3.073788 & 2.03 & -1.829276 \\
\hline $\mathrm{C}$ & 0.622069 & 9.174405 & 1.07 \\
\hline $\mathrm{C}$ & 3.2 & 5.8 & 3.6 \\
\hline C & 2.248233 & 3.266618 & 3.18 \\
\hline $\mathrm{C}$ & 4.08 & 4.46 & 1.31 \\
\hline $\mathrm{C}$ & 0.3 & 8.1 & 5.6 \\
\hline $\mathrm{C}$ & -2.491163 & 7.554692 & 5.941957 \\
\hline $\mathrm{C}$ & -0.5 & 5.35 & 5.8 \\
\hline $\mathrm{H}$ & -1.9 & 7.387077 & -1.1 \\
\hline $\mathrm{H}$ & -0.545809 & 2.830325 & -1.702703 \\
\hline $\mathrm{H}$ & 0.9 & 3.7 & -1.97 \\
\hline $\mathrm{H}$ & -0.539468 & 4.614094 & -1.550719 \\
\hline $\mathrm{H}$ & -4.035342 & 6.40 & 975 \\
\hline $\mathrm{H}$ & -4.952069 & 6.60 & 2.8 \\
\hline $\mathrm{H}$ & -5.064122 & 5.068693 & 1.983428 \\
\hline $\mathrm{H}$ & 0.6 & 8.7 & 0.06 \\
\hline $\mathrm{H}$ & 1.400632 & 9.930263 & 1.217274 \\
\hline $\mathrm{H}$ & -0.362222 & 9.628086 & 1.22 \\
\hline $\mathrm{H}$ & 4.11 & 5.3 & 4.21 \\
\hline $\mathrm{H}$ & 3.673537 & 6.832689 & 3.289274 \\
\hline $\mathrm{H}$ & 2.508721 & 6.109557 & 4.420948 \\
\hline $\mathrm{H}$ & 4.881196 & 4.016300 & 1.93 \\
\hline $\mathrm{H}$ & 3.796587 & 3.764694 & 0.526586 \\
\hline $\mathrm{H}$ & 4.461515 & 5.385114 & 0.854936 \\
\hline $\mathrm{H}$ & 3.110484 & 2.952948 & 3.790251 \\
\hline $\mathrm{H}$ & 1.371213 & 3.383830 & 3.835030 \\
\hline $\mathrm{H}$ & 2.023251 & 2.489552 & 2.447827 \\
\hline $\mathrm{H}$ & 0.337170 & 8.102330 & 6.790987 \\
\hline $\mathrm{H}$ & 1.286508 & 7.867913 & 5.289322 \\
\hline $\mathrm{H}$ & 0.072333 & 9.155958 & 5.368103 \\
\hline $\mathrm{H}$ & 0.419695 & 5.065953 & 5.536427 \\
\hline $\mathrm{H}$ & -0.558357 & 5.503469 & 6.963448 \\
\hline $\mathrm{H}$ & -1.309859 & 4.637998 & 5.588520 \\
\hline $\mathrm{H}$ & -2.801999 & 8.527209 & 5.543952 \\
\hline $\mathrm{H}$ & -3.296383 & 6.831242 & 5.774832 \\
\hline$\Pi$ & -2.299144 & 7.649209 & 7.019098 \\
\hline $\mathrm{H}$ & -4.176709 & 3.315386 & 1.101388 \\
\hline
\end{tabular}




$\begin{array}{rrrr}\mathrm{H} & -4.768831 & 2.409311 & 3.134125 \\ \mathrm{H} & -4.037757 & 1.359067 & 4.472433 \\ \mathrm{H} & -1.333441 & 0.644838 & 3.897663 \\ \mathrm{H} & -2.265710 & -0.619702 & 3.053629 \\ \mathrm{H} & -2.176945 & -0.093492 & 0.325566 \\ \mathrm{H} & -1.027671 & 1.235034 & -0.015422 \\ \mathrm{H} & -3.793883 & 5.125011 & -0.602949 \\ \mathrm{H} & -3.683517 & 5.671996 & -3.019930 \\ \mathrm{H} & -3.176532 & 3.891261 & -4.676497 \\ \mathrm{H} & -2.819828 & 1.556297 & -3.908230 \\ \mathrm{H} & -2.951166 & 1.004512 & -1.508608\end{array}$

\section{Def2-SVP (CPCM=toluene $)$}

\section{$\mathrm{HCOOH}$}

5

scf done: -189.524281

$\begin{array}{llll}\text { C } & 5.379909 & -1.330071 & 2.551851\end{array}$

$\begin{array}{llll}\mathrm{O} & 4.762460 & -0.961097 & 3.502307\end{array}$

$\begin{array}{llll}\text { O } & 5.489651 & -2.617073 & 2.215322\end{array}$

H $\quad 5.921465 \quad-0.643674 \quad 1.855553$

H $\quad 6.026511 \quad-2.709910 \quad 1.415341$

$\mathrm{PMe}_{3}$

13

scf done: -460.777944

$\begin{array}{llll}\text { C } & 2.317411 & 5.822961 & 3.090626\end{array}$

$\begin{array}{llll}\mathrm{P} & 2.589725 & 5.583155 & 1.274148\end{array}$

$\begin{array}{llll}\text { C } & 1.092469 & 6.478168 & 0.651298\end{array}$

$\begin{array}{llll}\text { C } & 3.846398 & 6.916714 & 1.002374\end{array}$

$\begin{array}{llll}\mathrm{H} & 3.520897 & 7.884294 & 1.424272\end{array}$

$\mathrm{H} \quad 4.802256 \quad 6.630149 \quad 1.469948$

$\begin{array}{llll}\mathrm{H} & 4.027680 & 7.044627 & -0.076965\end{array}$

$\mathrm{H} \quad 2.113540 \quad 6.879108 \quad 3.342287$

H $\quad \begin{array}{llll}1.465387 & 5.208771 & 3.423893\end{array}$

$\begin{array}{llll}\mathrm{H} & 3.207129 & 5.491519 & 3.649917\end{array}$

$\begin{array}{llll}\mathrm{H} & 0.992078 & 7.481769 & 1.101807\end{array}$

H $\quad 1.149220 \quad 6.585712 \quad-0.443784$

$\begin{array}{llll}\mathrm{H} & 0.187034 & 5.894172 & 0.882669\end{array}$

Enyne 1

25

scf done: -538.819326

C $\quad \begin{array}{llll}1.506750 & 3.894220 & -2.397494\end{array}$

$\begin{array}{llll}\text { C } & 2.182707 & 3.087376 & -3.328070\end{array}$

$\begin{array}{llll}\text { C } & 3.407788 & 3.546209 & -3.834592\end{array}$

C $\quad 3.935678 \quad 4.772491 \quad-3.440946$

$\begin{array}{llll}\text { C } & 3.249070 & 5.564849 & -2.523819\end{array}$

$\begin{array}{llll}\text { C } & 2.033590 & 5.117995 & -2.002413\end{array}$

$\begin{array}{llll}\text { C } & 1.670082 & 1.788313 & -3.766527\end{array}$

$\begin{array}{llll}\text { C } & 0.459632 & 1.274014 & -3.511910\end{array}$

C $\quad 0.031013 \quad-0.086386 \quad-3.944064$

$\begin{array}{llll}\text { O } & -0.403223 & -0.900181 & -2.875618\end{array}$ 


$\begin{array}{rrrr}\mathrm{C} & 0.579932 & -1.168178 & -1.913946 \\ \mathrm{C} & 0.733247 & -0.127724 & -0.894911 \\ \mathrm{C} & 0.865252 & 0.711484 & -0.031238 \\ \mathrm{H} & 0.985056 & 1.459929 & 0.730959 \\ \mathrm{H} & -0.832355 & -0.027982 & -4.629314 \\ \mathrm{H} & 0.858057 & -0.582849 & -4.496385 \\ \mathrm{H} & 1.565451 & -1.351934 & -2.393472 \\ \mathrm{H} & 0.295645 & -2.111832 & -1.416397 \\ \mathrm{H} & -0.280069 & 1.840820 & -2.931104 \\ \mathrm{H} & 2.373716 & 1.183421 & -4.358024 \\ \mathrm{H} & 3.950446 & 2.922702 & -4.553489 \\ \mathrm{H} & 4.891466 & 5.111398 & -3.851461 \\ \mathrm{H} & 3.661414 & 6.527882 & -2.209107 \\ \mathrm{H} & 1.492906 & 5.730493 & -1.274778 \\ \mathrm{H} & 0.560755 & 3.549226 & -1.968184\end{array}$

\section{Diene 3}

$$
25
$$

scf done: -538.879517

C $\quad-3.501701 \quad 2.448926 \quad-1.787561$

C $\quad-3.934215 \quad 2.954660 \quad-0.547383$

$\begin{array}{llll}\text { C } & -4.720627 & 4.121821 & -0.554762\end{array}$

$\begin{array}{llll}\text { C } & -5.035741 & 4.774493 & -1.739746\end{array}$

$\begin{array}{llll}\text { C } & -4.583053 & 4.268406 & -2.957809\end{array}$

$\begin{array}{llll}\text { C } & -3.822315 & 3.100434 & -2.974254\end{array}$

$\begin{array}{llll}\text { C } & -3.627551 & 2.340307 & 0.738561\end{array}$

$\begin{array}{llll}\text { C } & -2.683110 & 1.429695 & 1.049868\end{array}$

$\begin{array}{llll}\text { C } & -1.640118 & 0.804749 & 0.160482\end{array}$

$\begin{array}{llll}\mathrm{O} & -0.666864 & 0.272311 & 1.024264\end{array}$

$\begin{array}{llll}\text { C } & -1.303217 & -0.097763 & 2.222270\end{array}$

$\begin{array}{llll}\text { C } & -2.442784 & 0.872616 & 2.390203\end{array}$

$\begin{array}{llll}\text { C } & -3.077019 & 1.142030 & 3.536790\end{array}$

$\begin{array}{llll}\mathrm{H} & -4.245411 & 2.704231 & 1.570737\end{array}$

$\begin{array}{llll}\mathrm{H} & -3.909788 & 1.850413 & 3.587848\end{array}$

$\begin{array}{llll}\mathrm{H} & -2.780698 & 0.657709 & 4.472346\end{array}$

$\mathrm{H} \quad-0.574397 \quad-0.066081 \quad 3.048266$

$\mathrm{H} \quad-1.694610 \quad-1.137855 \quad 2.161565$

H $\quad-2.089913 \quad 0.004680 \quad-0.471804$

$\mathrm{H} \quad-1.160298 \quad 1.528800 \quad-0.521351$

$\mathrm{H} \quad-5.080323 \quad 4.521166 \quad 0.399706$

$\mathrm{H} \quad-5.642583 \quad 5.684338 \quad-1.714968$

H $\quad-4.830896 \quad 4.777842 \quad-3.893344$

$\mathrm{H} \quad-3.478304 \quad 2.685225 \quad-3.926184$

$\mathrm{H} \quad-2.933494 \quad 1.517007 \quad-1.834553$

\section{A}

57

scf done: -3079.942316

$\begin{array}{cccc}\mathrm{C} & 7.272893 & 1.980204 & -2.048083 \\ \mathrm{P} & 7.083969 & 0.872144 & -0.610862 \\ \mathrm{C} & 7.169251 & -0.798885 & -1.342415 \\ \mathrm{Pd} & 8.667198 & 1.456529 & 0.983303 \\ \mathrm{Pd} & 11.436561 & 1.459481 & 1.880460 \\ \mathrm{P} & 13.640525 & 0.836791 & 1.487769 \\ \mathrm{C} & 14.872623 & 1.121538 & 2.805852 \\ \mathrm{C} & 5.317475 & 1.036610 & -0.176544\end{array}$




$\begin{array}{cccc}\mathrm{S} & 10.456465 & 0.181625 & 0.174810 \\ \mathrm{C} & 10.197538 & -1.453459 & 0.960630 \\ \mathrm{Pd} & 9.497052 & 3.205660 & 3.162538 \\ \mathrm{~S} & 11.786656 & 3.012968 & 3.594539 \\ \mathrm{C} & 11.869792 & 1.978646 & 5.102794 \\ \mathrm{~S} & 7.386217 & 3.019805 & 2.162175 \\ \mathrm{C} & 6.424483 & 1.986318 & 3.328088 \\ \mathrm{P} & 9.029279 & 5.005879 & 4.553173 \\ \mathrm{C} & 13.967201 & -0.891877 & 0.996857 \\ \mathrm{C} & 14.278406 & 1.805662 & 0.078908 \\ \mathrm{H} & 11.668126 & 2.611952 & 5.979624 \\ \mathrm{H} & 12.886948 & 1.569363 & 5.197634 \\ \mathrm{H} & 11.143040 & 1.155620 & 5.067554 \\ \mathrm{H} & 5.587411 & 1.523581 & 2.783988 \\ \mathrm{H} & 6.009700 & 2.629125 & 4.118804 \\ \mathrm{H} & 7.048515 & 1.202506 & 3.778934 \\ \mathrm{H} & 11.130541 & -2.033764 & 0.902529 \\ \mathrm{H} & 9.418621 & -1.996067 & 0.404601 \\ \mathrm{H} & 9.893804 & -1.350030 & 2.011415 \\ \mathrm{H} & 15.041477 & -1.034300 & 0.792459 \\ \mathrm{H} & 13.662619 & -1.576571 & 1.803588 \\ \mathrm{H} & 13.391335 & -1.133582 & 0.089971 \\ \mathrm{H} & 15.884469 & 0.871564 & 2.445013 \\ \mathrm{H} & 14.847316 & 2.176746 & 3.118965 \\ \mathrm{H} & 14.640478 & 0.488484 & 3.676234 \\ \mathrm{H} & 15.325931 & 1.540725 & -0.141601 \\ \mathrm{H} & 13.655357 & 1.605865 & -0.807023 \\ \mathrm{H} & 14.214406 & 2.879667 & 0.311800 \\ \mathrm{H} & 4.685610 & 0.774722 & -1.041794 \\ \mathrm{H} & 5.070130 & 0.366269 & 0.661281 \\ \mathrm{H} & 5.105842 & 2.074622 & 0.123843 \\ \mathrm{H} & 7.004066 & -1.560820 & -0.564913 \\ \mathrm{H} & 6.398283 & -0.913096 & -2.122393 \\ \mathrm{H} & 8.162291 & -0.956298 & -1.791560 \\ \mathrm{H} & 7.152332 & 3.024535 & -1.720104 \\ \mathrm{H} & 8.284772 & 1.863486 & -2.466791 \\ \mathrm{H} & 6.524759 & 1.750291 & -2.824878 \\ \mathrm{C} & 10.077912 & 5.265629 & 6.026038 \\ \mathrm{C} & 9.256979 & 6.544364 & 3.598245 \\ \mathrm{C} & 7.337362 & 5.165261 & 5.223557 \\ \mathrm{H} & 7.236715 & 6.110901 & 5.781925 \\ \mathrm{H} & 6.609463 & 5.155387 & 4.397520 \\ \mathrm{H} & 7.118094 & 4.325922 & 5.901682 \\ \mathrm{H} & 9.043846 & 7.429580 & 4.220624 \\ \mathrm{H} & 10.294704 & 6.593592 & 3.232858 \\ \mathrm{H} & 8.582736 & 6.535866 & 2.727704 \\ \mathrm{H} & 9.784376 & 6.194278 & 6.543363 \\ \mathrm{H} & 9.970078 & 4.420107 & 6.722560 \\ \mathrm{H} & 11.133080 & 5.341228 & 5.720485\end{array}$

\begin{tabular}{|c|c|c|c|}
\hline \\
\hline \multicolumn{4}{|c|}{ 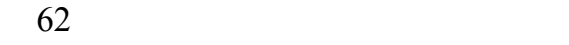 } \\
\hline $\mathrm{C}$ & 7.911356 & 1.818211 & -2.137936 \\
\hline 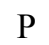 & 7.120544 & 1.393554 & -0.551703 \\
\hline $\mathrm{Pd}$ & 6.812853 & -0.912733 & -0.418239 \\
\hline $\mathrm{S}$ & 4.806177 & -1.039423 & -1.632068 \\
\hline C & 3.426137 & -0.564560 & -0.527989 \\
\hline
\end{tabular}




\begin{tabular}{|c|c|c|c|}
\hline $\mathrm{C}$ & 8.185416 & 2.178343 & 5973 \\
\hline $\mathrm{C}$ & 5.622211 & 2.434912 & -0.511791 \\
\hline Pd & 7.818200 & -3.545120 & 0.286571 \\
\hline $\mathrm{S}$ & 6.310226 & -5.220399 & -0.334506 \\
\hline $\mathrm{C}$ & 5.422288 & -5.689430 & 1.196127 \\
\hline $\mathrm{S}$ & 8.886548 & -1.475184 & 0.522827 \\
\hline $\mathrm{C}$ & 8.820908 & -1.103797 & 2.313603 \\
\hline $\mathrm{Pd}$ & 5.256520 & -3.276100 & -1.089613 \\
\hline$P$ & 3.339863 & -4.240720 & -1.988777 \\
\hline $\mathrm{C}$ & 1.899091 & -4.158902 & -0.869871 \\
\hline $\mathrm{P}$ & 9.359537 & -4.879068 & 1.401501 \\
\hline $\mathrm{C}$ & 2.739923 & -3.486668 & -3.538027 \\
\hline $\mathrm{C}$ & 3.456226 & -6.011660 & -2.413698 \\
\hline $\mathrm{O}$ & 5.393510 & -2.354611 & 2.358345 \\
\hline $\mathrm{C}$ & 5.189631 & -1.087915 & 2.729259 \\
\hline $\mathrm{O}$ & 4.656891 & -0.783909 & 3.751646 \\
\hline $\mathrm{H}$ & 6.017821 & -6.446665 & 1.729428 \\
\hline $\mathrm{H}$ & 4.461494 & -6.148714 & 0.917447 \\
\hline $\mathrm{H}$ & 5.242255 & -4.826599 & 1.853212 \\
\hline $\mathrm{H}$ & 8.732873 & -0.016510 & 2.454441 \\
\hline $\mathrm{H}$ & 9.765669 & -1.431960 & 2.773942 \\
\hline $\mathrm{H}$ & 7.978204 & -1.607090 & 2.808504 \\
\hline $\mathrm{H}$ & 2.481412 & -0.684722 & -1.080370 \\
\hline $\mathrm{H}$ & 3.530094 & 0.494700 & -0.250211 \\
\hline $\mathrm{H}$ & 3.394664 & -1.180808 & 0.381710 \\
\hline $\mathrm{H}$ & 1.027399 & -4.651772 & -1.332161 \\
\hline $\mathrm{H}$ & 2.135130 & -4.658060 & 0.082934 \\
\hline $\mathrm{H}$ & 1.647484 & -3.108465 & -0.657538 \\
\hline $\mathrm{H}$ & 2.507450 & -6.361004 & -2.853908 \\
\hline $\mathrm{H}$ & 4.272990 & -6.163928 & -3.135536 \\
\hline $\mathrm{H}$ & 3.677671 & -6.603702 & -1.512985 \\
\hline $\mathrm{H}$ & 1.841496 & -4.015499 & -3.897255 \\
\hline $\mathrm{H}$ & 2.500780 & -2.425105 & -3.374832 \\
\hline $\mathrm{H}$ & 3.528902 & -3.546632 & -4.303342 \\
\hline $\mathrm{H}$ & 8.289302 & 3.256148 & 0.498493 \\
\hline $\mathrm{H}$ & 7.736027 & 2.054613 & 1.704619 \\
\hline $\mathrm{H}$ & 9.183081 & 1.712520 & 0.699753 \\
\hline $\mathrm{H}$ & 5.077653 & 2.273465 & 0.432706 \\
\hline $\mathrm{H}$ & 5.895710 & 3.500669 & -0.585287 \\
\hline $\mathrm{H}$ & 4.964872 & 2.175416 & -1.356283 \\
\hline $\mathrm{H}$ & 8.887487 & 1.313278 & -2.205498 \\
\hline $\mathrm{H}$ & 7.277405 & 1.461540 & -2.964727 \\
\hline $\mathrm{H}$ & 8.053496 & 2.907908 & -2.227769 \\
\hline $\mathrm{H}$ & 5.577365 & -0.348017 & 1.975653 \\
\hline $\mathrm{H}$ & 5.838242 & -2.390957 & 1.477621 \\
\hline $\mathrm{C}$ & 9.426177 & -6.632761 & 0.902652 \\
\hline $\mathrm{C}$ & 11.102560 & -4.349890 & 1.292504 \\
\hline $\mathrm{C}$ & 9.013362 & -4.940218 & 3.193883 \\
\hline $\mathrm{H}$ & 9.720148 & -5.613360 & 3.707381 \\
\hline $\mathrm{H}$ & 9.097601 & -3.931239 & 3.625716 \\
\hline $\mathrm{H}$ & 7.985102 & -5.297094 & 3.360860 \\
\hline $\mathrm{H}$ & 11.752909 & -5.040822 & 1.853856 \\
\hline $\mathrm{H}$ & 11.412396 & -4.334798 & 0.236373 \\
\hline $\mathrm{H}$ & 11.214507 & -3.332663 & 1.697166 \\
\hline $\mathrm{H}$ & 10.194960 & -7.169061 & 1.483135 \\
\hline $\mathrm{H}$ & 8.447854 & -7.109536 & 1.067057 \\
\hline $\mathrm{H}$ & 9.664487 & -6.699925 & -0.169773 \\
\hline
\end{tabular}




\section{II \\ 74}

scf done: -3347.521202

C $12.864394 \quad 4.036683 \quad 0.931916$

$\begin{array}{llll}\text { C } & 11.681289 & 4.419709 & 0.277265\end{array}$

$\begin{array}{llll}\text { C } & 11.285815 & 3.689416 & -0.854632\end{array}$

C $12.044822 \quad 2.622764 \quad-1.327577$

C $\quad 13.221545 \quad 2.261924 \quad-0.675057$

$\begin{array}{llll}\text { C } & 13.625786 & 2.973928 & 0.455344\end{array}$

$\begin{array}{llll}\text { C } & 10.866117 & 5.561654 & 0.694211\end{array}$

$\begin{array}{llll}\text { C } & 11.026805 & 6.347402 & 1.773160\end{array}$

C $\quad 10.269592 \quad 7.620294 \quad 1.956984$

$\begin{array}{llll}\text { O } & 9.819987 & 7.866497 & 3.270563\end{array}$

$\begin{array}{llll}\text { C } & 8.525738 & 7.458645 & 3.582121\end{array}$

$\begin{array}{llll}\text { C } & 8.416358 & 6.123337 & 4.184630\end{array}$

$\begin{array}{llll}\text { C } & 8.172029 & 5.119870 & 4.857837\end{array}$

$\begin{array}{llll}\mathrm{Pd} & 9.279853 & 4.227707 & 3.117033\end{array}$

$\begin{array}{llll}\text { S } & 11.204975 & 3.701104 & 4.374757\end{array}$

C $\quad 10.640113 \quad 3.020497 \quad 5.972044$

$\begin{array}{llll}\mathrm{Pd} & 8.776207 & 2.208550 & 1.094247\end{array}$

$\begin{array}{llll}\text { Pd } & 11.093197 & 1.964989 & 2.838579\end{array}$

$\begin{array}{llll}\mathrm{P} & 12.892278 & 0.717284 & 3.573426\end{array}$

$\begin{array}{llll}\text { C } & 14.267619 & 1.689205 & 4.276862\end{array}$

$\begin{array}{llll}\mathrm{P} & 7.504052 & 1.308858 & -0.604007\end{array}$

$\begin{array}{llll}\text { C } & 7.311259 & -0.503477 & -0.615873\end{array}$

$\begin{array}{llll}\text { C } & 8.219708 & 1.695158 & -2.235683\end{array}$

$\begin{array}{llll}\text { C } & 5.784504 & 1.901592 & -0.713507\end{array}$

$\begin{array}{llll}\text { S } & 10.398710 & 0.505034 & 1.121568\end{array}$

$\begin{array}{llll}\text { C } & 9.702017 & -0.889430 & 2.078673\end{array}$

$\begin{array}{llll}\mathrm{S} & 7.665731 & 4.237846 & 1.377713\end{array}$

$\begin{array}{llll}\text { C } & 6.038753 & 3.947285 & 2.152573\end{array}$

C $\quad 12.449195 \quad-0.450030 \quad 4.905138$

$\begin{array}{llll}\text { C } & 13.722443 & -0.327880 & 2.328661\end{array}$

$\begin{array}{llll}\mathrm{O} & 7.789522 & 1.365924 & 4.336100\end{array}$

C $\quad 6.871026 \quad 0.567214 \quad 3.814953$

$\begin{array}{llll}\mathrm{O} & 6.553873 & 0.524951 & 2.655950\end{array}$

H $\quad 10.298156 \quad 3.857803 \quad 6.599297$

$\mathrm{H} \quad 11.491240 \quad 2.540738 \quad 6.479699$

$\begin{array}{llll}\mathrm{H} & 9.826725 & 2.292328 & 5.840522\end{array}$

H $\quad 5.254189 \quad 4.066539 \quad 1.390663$

H $\quad 5.886991 \quad 4.709182 \quad 2.932224$

$\begin{array}{llll}\mathrm{H} & 5.970200 & 2.941705 & 2.590497\end{array}$

H $\quad 10.500239 \quad-1.630296 \quad 2.242773$

H $\quad 8.898510 \quad-1.369420 \quad 1.501494$

H $\quad 9.305391 \quad-0.555424 \quad 3.047515$

$\mathrm{H} \quad 7.871033 \quad 4.399856 \quad 5.605686$

$\begin{array}{llll}\mathrm{H} & 10.944200 & 8.462841 & 1.714671\end{array}$

H $\quad 9.426539 \quad 7.672513 \quad 1.238014$

$\begin{array}{llll}\mathrm{H} & 7.860187 & 7.503208 & 2.694767\end{array}$

H $\quad 8.115788 \quad 8.174734 \quad 4.317553$

H $\quad 11.823959 \quad 6.157687 \quad 2.505490$

H $\quad 10.048997 \quad 5.814928 \quad 0.002130$

$\begin{array}{llll}\mathrm{H} & 13.332709 & -1.023123 & 5.232349\end{array}$

H $\quad 12.039571 \quad 0.103739 \quad 5.764102$

H $\quad 11.677891 \quad-1.148823 \quad 4.546561$

H $\quad 15.107742 \quad 1.027585 \quad 4.545478$

$\mathrm{H} \quad 14.610217 \quad 2.430687 \quad 3.538486$

$\mathrm{H} \quad 13.928651 \quad 2.232583 \quad 5.171933$

H $14.586880 \quad-0.841498 \quad 2.781029$

H $13.022324 \quad-1.077748 \quad 1.930559$ 


$\begin{array}{cccc}\mathrm{H} & 14.064757 & 0.301412 & 1.492131 \\ \mathrm{H} & 5.264350 & 1.399146 & -1.545700 \\ \mathrm{H} & 5.259973 & 1.676707 & 0.227935 \\ \mathrm{H} & 5.775537 & 2.989281 & -0.881960 \\ \mathrm{H} & 6.824973 & -0.821560 & 0.319620 \\ \mathrm{H} & 6.683191 & -0.805535 & -1.470399 \\ \mathrm{H} & 8.294893 & -0.990827 & -0.704126 \\ \mathrm{H} & 8.290369 & 2.787978 & -2.354646 \\ \mathrm{H} & 9.234105 & 1.269560 & -2.294374 \\ \mathrm{H} & 7.598670 & 1.279503 & -3.046554 \\ \mathrm{H} & 6.422780 & -0.070432 & 4.607667 \\ \mathrm{H} & 8.210402 & 1.916959 & 3.625386 \\ \mathrm{H} & 10.368529 & 3.984325 & -1.378841 \\ \mathrm{H} & 11.723087 & 2.076134 & -2.219298 \\ \mathrm{H} & 13.830285 & 1.435246 & -1.053309 \\ \mathrm{H} & 14.562601 & 2.712052 & 0.959345 \\ \mathrm{H} & 13.210687 & 4.596160 & 1.807731\end{array}$

\section{TS (II-III)}

74

scf done: -3347.457026

$\begin{array}{llll}\text { C } & 0.136540 & -0.098852 & -0.008031\end{array}$

$\begin{array}{llll}\text { C } & 0.162253 & -0.142935 & 1.397927\end{array}$

$\begin{array}{llll}\text { C } & 1.413020 & -0.147878 & 2.035395\end{array}$

$\begin{array}{llll}\text { C } & 2.597218 & -0.114119 & 1.304095\end{array}$

$\begin{array}{llll}\text { C } & 2.555735 & -0.080119 & -0.087980\end{array}$

C $\quad 1.319714 \quad-0.074331 \quad-0.738573$

$\begin{array}{llll}\text { C } & -1.048223 & -0.202514 & 2.220420\end{array}$

$\begin{array}{llll}\text { C } & -2.319144 & -0.235244 & 1.790593\end{array}$

$\begin{array}{llll}\text { C } & -3.491323 & -0.346977 & 2.710591\end{array}$

$\begin{array}{llll}\text { O } & -4.312849 & 0.800809 & 2.717611\end{array}$

$\begin{array}{llll}\text { C } & -5.086761 & 1.024522 & 1.571013\end{array}$

$\begin{array}{llll}\text { C } & -4.983261 & 2.424211 & 1.088424\end{array}$

$\begin{array}{llll}\text { C } & -5.602582 & 3.495643 & 0.773429\end{array}$

$\begin{array}{llll}\mathrm{Pd} & -2.929552 & 2.757079 & 0.846584\end{array}$

$\begin{array}{llll}\text { S } & -2.397139 & 3.963312 & 2.910792\end{array}$

$\begin{array}{llll}\text { C } & -3.864923 & 4.905448 & 3.440684\end{array}$

$\begin{array}{llll}\mathrm{Pd} & -1.352094 & 5.243612 & 1.234697\end{array}$

$\begin{array}{llll}\mathrm{O} & -3.107687 & 6.480152 & 0.791227\end{array}$

$\begin{array}{llll}\text { C } & -4.042532 & 6.556499 & -0.008019\end{array}$

$\begin{array}{llll}\mathrm{O} & -4.758296 & 5.616614 & -0.485233\end{array}$

$\begin{array}{llll}\mathrm{Pd} & -1.637674 & 3.976347 & -1.416220\end{array}$

$\begin{array}{llll}\mathrm{P} & -0.889396 & 3.838048 & -3.556639\end{array}$

$\begin{array}{llll}\text { C } & -0.220208 & 5.371792 & -4.271329\end{array}$

$\begin{array}{llll}\text { S } & -2.832719 & 1.969221 & -1.423517\end{array}$

C $\quad-4.444909 \quad 2.311439 \quad-2.206293$

$\begin{array}{llll}\text { P } & 0.662325 & 4.353282 & 1.814326\end{array}$

$\begin{array}{llll}\text { C } & 1.839187 & 5.668806 & 2.247394\end{array}$

$\begin{array}{llll}\text { S } & -0.325304 & 5.928497 & -0.844589\end{array}$

C $\quad-1.228244 \quad 7.391091 \quad-1.461357$

$\begin{array}{llll}\text { C } & 0.708469 & 3.195958 & 3.212945\end{array}$

$\begin{array}{llll}\text { C } & 1.452335 & 3.424416 & 0.465273\end{array}$

C $\quad-2.088691 \quad 3.265585 \quad-4.798613$

$\begin{array}{llll}\text { C } & 0.471114 & 2.633307 & -3.641715\end{array}$

$\mathrm{H} \quad-6.659935 \quad 3.744993 \quad 0.934829$

$\begin{array}{llll}\mathrm{H} & -4.942072 & 4.674428 & 0.092920\end{array}$

$\begin{array}{llll}\mathrm{H} & -5.134388 & 1.506497 & -1.908005\end{array}$

$\begin{array}{llll}\mathrm{H} & -4.335464 & 2.286747 & -3.300032\end{array}$ 


$\begin{array}{rrrr}\mathrm{H} & -4.866313 & 3.274645 & -1.883711 \\ \mathrm{H} & -4.765762 & 4.319838 & 3.204064 \\ \mathrm{H} & -3.917226 & 5.877652 & 2.934707 \\ \mathrm{H} & -3.792557 & 5.058059 & 4.526511 \\ \mathrm{H} & -2.131884 & 7.099242 & -2.016872 \\ \mathrm{H} & -0.554022 & 7.943686 & -2.132119 \\ \mathrm{H} & -1.509673 & 8.038607 & -0.619673 \\ \mathrm{H} & 0.124047 & 5.178954 & -5.300627 \\ \mathrm{H} & -1.002745 & 6.146005 & -4.291856 \\ \mathrm{H} & 0.621578 & 5.735020 & -3.662389 \\ \mathrm{H} & -1.620317 & 3.278330 & -5.796781 \\ \mathrm{H} & -2.408316 & 2.239254 & -4.562951 \\ \mathrm{H} & -2.970042 & 3.924987 & -4.806167 \\ \mathrm{H} & 0.867962 & 2.569114 & -4.668482 \\ \mathrm{H} & 1.279427 & 2.931541 & -2.955981 \\ \mathrm{H} & 0.089776 & 1.648293 & -3.330069 \\ \mathrm{H} & 2.819799 & 5.225231 & 2.487470 \\ \mathrm{H} & 1.944263 & 6.360094 & 1.398117 \\ \mathrm{H} & 1.467198 & 6.228737 & 3.118151 \\ \mathrm{H} & 1.650667 & 4.087967 & -0.389995 \\ \mathrm{H} & 2.402611 & 2.996612 & 0.826234 \\ \mathrm{H} & 0.788765 & 2.602727 & 0.144601 \\ \mathrm{H} & 0.331211 & 3.676139 & 4.126660 \\ \mathrm{H} & 0.084055 & 2.315768 & 2.989036 \\ \mathrm{H} & 1.752068 & 2.870887 & 3.360371 \\ \mathrm{H} & -4.322098 & 7.564191 & -0.393762 \\ \mathrm{H} & -6.162274 & 0.857517 & 1.793827 \\ \mathrm{H} & -4.826236 & 0.319508 & 0.754957 \\ \mathrm{H} & -3.144841 & -0.479165 & 3.747934 \\ \mathrm{H} & -4.094259 & -1.242737 & 2.452228 \\ \mathrm{H} & -2.530344 & -0.216396 & 0.712283 \\ \mathrm{H} & -0.873363 & -0.243305 & 3.305982 \\ \mathrm{H} & 1.448720 & -0.189671 & 3.129806 \\ \mathrm{H} & 3.559031 & -0.130501 & 1.824962 \\ \mathrm{H} & 3.482892 & -0.071324 & -0.667929 \\ \mathrm{H} & 1.280968 & -0.066557 & -1.831985 \\ \mathrm{H} & -0.823135 & -0.097195 & -0.537938\end{array}$

III

74

scf done: -3347.524937

$\begin{array}{rrrr}\text { C } & 1.934125 & -0.641203 & -3.632227 \\ \mathrm{C} & 2.561020 & -1.323752 & -2.578134 \\ \mathrm{C} & 2.455289 & -2.706214 & -2.449583 \\ \mathrm{C} & 1.711347 & -3.438187 & -3.373049 \\ \mathrm{C} & 1.072319 & -2.773128 & -4.421536 \\ \mathrm{C} & 1.182584 & -1.392571 & -4.551933 \\ \mathrm{C} & 2.075966 & 0.814564 & -3.722127 \\ \mathrm{C} & 1.677550 & 1.601317 & -4.731727 \\ \mathrm{C} & 1.786685 & 3.090647 & -4.710740 \\ \mathrm{O} & 2.483348 & 3.626235 & -5.810407 \\ \mathrm{C} & 1.827134 & 3.565909 & -7.047963 \\ \mathrm{C} & 2.742817 & 3.081007 & -8.123723 \\ \mathrm{Pd} & 3.485070 & 1.257499 & -7.868436 \\ \mathrm{Pd} & 6.177954 & 0.129737 & -7.364827 \\ \mathrm{P} & 5.998917 & -1.141153 & -5.487669 \\ \mathrm{C} & 5.825733 & -0.268456 & -3.902608 \\ \mathrm{C} & 3.140022 & 3.779196 & -9.183326\end{array}$




\begin{tabular}{|c|c|c|c|}
\hline $\mathrm{Pd}$ & 4.322541 & -0.985449 & -9.577921 \\
\hline & 5.290184 & 0.696517 & -10.528762 \\
\hline $\mathrm{C}$ & 6.223090 & 1.413212 & -10.105501 \\
\hline $\mathrm{O}$ & 6.759014 & 1.398710 & -8.976040 \\
\hline $\mathrm{S}$ & 2.215454 & 0.174786 & -9.602425 \\
\hline $\mathrm{C}$ & 2.251161 & 1.169044 & -11.125269 \\
\hline $\mathrm{S}$ & 5.090937 & 1.937965 & -6.239066 \\
\hline $\mathrm{C}$ & 5.822846 & 3.487878 & -6.838433 \\
\hline $\mathrm{P}$ & 3.129000 & -2.895241 & -9.281968 \\
\hline $\mathrm{C}$ & 2.258718 & -3.243557 & -10.843546 \\
\hline $\mathrm{S}$ & 6.398891 & -1.892198 & -8.727860 \\
\hline $\mathrm{C}$ & 7.685836 & -1.562992 & -9.981814 \\
\hline $\mathrm{C}$ & 1.823214 & -2.888230 & -8.016291 \\
\hline $\mathrm{C}$ & 4.075951 & -4.418106 & -8.966025 \\
\hline $\mathrm{C}$ & 4.609127 & -2.311039 & -5.495061 \\
\hline $\mathrm{C}$ & 7.491101 & -2.163171 & -5.289003 \\
\hline $\mathrm{H}$ & 2.803925 & 4.817677 & -9.317030 \\
\hline $\mathrm{H}$ & 3.805835 & 3.372780 & -9.954578 \\
\hline $\mathrm{H}$ & 1.581052 & 2.030381 & -10.988296 \\
\hline $\mathrm{H}$ & 1.880308 & 0.546268 & -11.952419 \\
\hline $\mathrm{H}$ & 3.271375 & 1.513536 & -11.343210 \\
\hline $\mathrm{H}$ & 5.991298 & 3.467506 & -7.922140 \\
\hline $\mathrm{H}$ & 6.770265 & 3.665263 & -6.310330 \\
\hline $\mathrm{H}$ & 5.100562 & 4.278236 & -6.584230 \\
\hline $\mathrm{H}$ & 7.263837 & -1.697262 & -10.987751 \\
\hline $\mathrm{H}$ & 8.495333 & -2.290747 & -9.829369 \\
\hline $\mathrm{H}$ & 8.086978 & -0.545994 & -9.881730 \\
\hline $\mathrm{H}$ & 3.390528 & -5.280811 & -8.989744 \\
\hline $\mathrm{H}$ & 4.848751 & -4.539044 & -9.739708 \\
\hline $\mathrm{H}$ & 4.576505 & -4.382974 & -7.987159 \\
\hline $\mathrm{H}$ & 1.712702 & -4.198427 & -10.765004 \\
\hline $\mathrm{H}$ & 1.550174 & -2.428670 & -11.054554 \\
\hline $\mathrm{H}$ & 2.984218 & -3.307447 & -11.668722 \\
\hline $\mathrm{H}$ & 1.250603 & -3.828645 & -8.076653 \\
\hline $\mathrm{H}$ & 2.259670 & -2.798512 & -7.009691 \\
\hline $\mathrm{H}$ & 1.145519 & -2.038247 & -8.191209 \\
\hline $\mathrm{H}$ & 7.424215 & -2.748374 & -4.357340 \\
\hline $\mathrm{H}$ & 7.586573 & -2.842373 & -6.149054 \\
\hline $\mathrm{H}$ & 8.380843 & -1.516755 & -5.248897 \\
\hline $\mathrm{H}$ & 4.668124 & -2.956991 & -6.384642 \\
\hline $\mathrm{H}$ & 4.625562 & -2.936894 & -4.586471 \\
\hline $\mathrm{H}$ & 3.662453 & -1.742948 & -5.508577 \\
\hline $\mathrm{H}$ & 6.639588 & 0.462271 & -3.783967 \\
\hline $\mathrm{H}$ & 4.866472 & 0.272814 & -3.876815 \\
\hline $\mathrm{H}$ & 5.859756 & -1.004288 & -3.082152 \\
\hline $\mathrm{H}$ & 6.631565 & 2.158854 & -10.827831 \\
\hline $\mathrm{H}$ & 1.467219 & 4.577030 & -7.338863 \\
\hline $\mathrm{H}$ & 0.928411 & 2.918422 & -7.002366 \\
\hline $\mathrm{H}$ & 2.327684 & 3.415172 & -3.806493 \\
\hline $\mathrm{H}$ & 0.769844 & 3.536491 & -4.652487 \\
\hline $\mathrm{H}$ & 1.216248 & 1.153413 & -5.625017 \\
\hline $\mathrm{H}$ & 2.550745 & 1.292681 & -2.852072 \\
\hline $\mathrm{H}$ & 3.138433 & -0.748554 & -1.845434 \\
\hline $\mathrm{H}$ & 2.947400 & -3.214644 & -1.615239 \\
\hline $\mathrm{H}$ & 1.613388 & -4.522164 & -3.267412 \\
\hline $\mathrm{H}$ & 0.465506 & -3.337958 & -5.136331 \\
\hline $\mathrm{H}$ & 0.658782 & -0.884055 & -5.369227 \\
\hline
\end{tabular}




\begin{tabular}{|c|c|c|c|}
\hline \multicolumn{4}{|c|}{$\begin{array}{l}\text { TS (I-IV) } \\
62\end{array}$} \\
\hline $\mathrm{C}$ & 0.534764 & 0.967038 & -0.397857 \\
\hline $\mathrm{P}$ & 0.333130 & 0.705381 & 1.394737 \\
\hline $\mathrm{C}$ & 2.004151 & 0.905441 & 2.085969 \\
\hline $\mathrm{Pd}$ & -1.163953 & 2.121969 & 2.394395 \\
\hline $\mathrm{S}$ & -2.467333 & 0.299409 & 3.142311 \\
\hline $\mathrm{C}$ & -3.462721 & 0.917783 & 4.543803 \\
\hline $\mathrm{S}$ & -0.338009 & 3.858125 & 1.015191 \\
\hline $\mathrm{C}$ & -0.702562 & 5.419781 & 1.887076 \\
\hline $\mathrm{Pd}$ & -2.441986 & 3.260924 & -0.115025 \\
\hline $\mathrm{S}$ & -4.351154 & 2.113978 & -0.838630 \\
\hline $\mathrm{C}$ & -5.760680 & 3.170454 & -0.346939 \\
\hline $\mathrm{P}$ & -1.921331 & 4.554315 & -2.005093 \\
\hline $\mathrm{C}$ & -0.750523 & 3.706671 & -3.121122 \\
\hline $\mathrm{C}$ & -3.326353 & 4.985572 & -3.087625 \\
\hline $\mathrm{C}$ & -1.109940 & 6.161368 & -1.698958 \\
\hline $\mathrm{Pd}$ & -3.701847 & 1.047020 & 1.146324 \\
\hline $\mathrm{P}$ & -4.982991 & -0.925475 & 1.127346 \\
\hline $\mathrm{C}$ & -6.280719 & -1.048547 & -0.151205 \\
\hline $\mathrm{O}$ & -3.948759 & 3.795276 & 2.488601 \\
\hline $\mathrm{C}$ & -3.230933 & 4.014551 & 3.494268 \\
\hline $\mathrm{O}$ & -2.111622 & 3.519530 & 3.745265 \\
\hline $\mathrm{C}$ & 0.001670 & -1.083036 & 1.497546 \\
\hline $\mathrm{C}$ & -3.981548 & -2.429138 & 0.856398 \\
\hline $\mathrm{C}$ & -5.877260 & -1.287156 & 2.678664 \\
\hline $\mathrm{H}$ & -3.258177 & 2.859633 & 1.576318 \\
\hline $\mathrm{H}$ & -0.167951 & 6.228402 & 1.366948 \\
\hline $\mathrm{H}$ & -0.346491 & 5.354462 & 2.923947 \\
\hline $\mathrm{H}$ & -1.780854 & 5.632306 & 1.883950 \\
\hline $\mathrm{H}$ & -5.581315 & 3.647998 & 0.626122 \\
\hline $\mathrm{H}$ & -6.663960 & 2.544343 & -0.298947 \\
\hline $\mathrm{H}$ & -5.909642 & 3.936724 & -1.122314 \\
\hline $\mathrm{H}$ & -2.820820 & 1.490055 & 5.227006 \\
\hline $\mathrm{H}$ & -3.870246 & 0.045864 & 5.075991 \\
\hline $\mathrm{H}$ & -4.288623 & 1.555561 & 4.196176 \\
\hline $\mathrm{H}$ & 0.816549 & -1.628634 & 0.993883 \\
\hline $\mathrm{H}$ & -0.066303 & -1.407523 & 2.545942 \\
\hline $\mathrm{H}$ & -0.948915 & -1.315561 & 0.991147 \\
\hline $\mathrm{H}$ & 2.714318 & 0.225356 & 1.587217 \\
\hline $\mathrm{H}$ & 2.327498 & 1.948118 & 1.947005 \\
\hline $\mathrm{H}$ & 1.982430 & 0.687980 & 3.164378 \\
\hline $\mathrm{H}$ & 1.278293 & 0.259014 & -0.798534 \\
\hline $\mathrm{H}$ & -0.435356 & 0.809509 & -0.897763 \\
\hline $\mathrm{H}$ & 0.870827 & 1.997016 & -0.593504 \\
\hline $\mathrm{H}$ & -6.525662 & -2.170017 & 2.554232 \\
\hline $\mathrm{H}$ & -5.151601 & -1.488391 & 3.481429 \\
\hline $\mathrm{H}$ & -6.493872 & -0.422657 & 2.969151 \\
\hline $\mathrm{H}$ & -3.227419 & -2.514288 & 1.654834 \\
\hline $\mathrm{H}$ & -4.618599 & -3.329234 & 0.860478 \\
\hline $\mathrm{H}$ & -3.464204 & -2.358948 & -0.113326 \\
\hline $\mathrm{H}$ & -7.024464 & -0.250279 & -0.004998 \\
\hline $\mathrm{H}$ & -5.828681 & -0.924088 & -1.147054 \\
\hline $\mathrm{H}$ & -6.787321 & -2.026484 & -0.096469 \\
\hline $\mathrm{H}$ & -3.637707 & 4.732830 & 4.247507 \\
\hline $\mathrm{H}$ & -0.944702 & 6.696371 & -2.648926 \\
\hline $\mathrm{H}$ & -1.734383 & 6.781766 & -1.037552 \\
\hline $\mathrm{H}$ & -0.138382 & 5.989810 & -1.209647 \\
\hline $\mathrm{H}$ & -2.982869 & 5.555899 & -3.966331 \\
\hline
\end{tabular}




$\begin{array}{crrr}\mathrm{H} & -3.830421 & 4.065349 & -3.421590 \\ \mathrm{H} & -4.050425 & 5.595083 & -2.525035 \\ \mathrm{H} & -0.518100 & 4.336073 & -3.996268 \\ \mathrm{H} & 0.181051 & 3.492542 & -2.573502 \\ \mathrm{H} & -1.183639 & 2.753772 & -3.463369\end{array}$

\section{IV}

62

scf done: -3269.455449

$\begin{array}{llll}\text { C } & -0.027279 & -0.036977 & 0.007940\end{array}$

$\begin{array}{llll}\mathrm{P} & 0.025337 & -0.069982 & 1.828850\end{array}$

$\begin{array}{llll}\text { C } & 1.811939 & -0.201072 & 2.178188\end{array}$

$\begin{array}{llll}\text { C } & -0.413400 & 1.626299 & 2.323321\end{array}$

$\begin{array}{llll}\text { Pd } & -1.302405 & -1.622572 & 2.895791\end{array}$

$\begin{array}{llll}\mathrm{O} & -2.883739 & -2.560293 & 3.945334\end{array}$

$\begin{array}{llll}\text { C } & -2.892062 & -3.765381 & 4.359091\end{array}$

$\begin{array}{llll}\mathrm{O} & -2.024279 & -4.625453 & 4.236350\end{array}$

$\begin{array}{llll}\text { S } & -0.051755 & -1.197324 & 4.887883\end{array}$

$\begin{array}{llll}\mathrm{Pd} & 1.045007 & -3.226203 & 4.031029\end{array}$

$\begin{array}{llll}\mathrm{P} & 2.812136 & -3.060584 & 5.544230\end{array}$

$\begin{array}{llll}\text { C } & 2.277602 & -3.149220 & 7.285249\end{array}$

$\begin{array}{llll}\text { S } & -1.937644 & -2.580473 & 0.806629\end{array}$

$\begin{array}{llll}\mathrm{Pd} & -0.113335 & -4.061802 & 1.522667\end{array}$

$\begin{array}{llll}\mathrm{P} & 0.010444 & -5.078929 & -0.565050\end{array}$

$\begin{array}{llll}\text { C } & -1.564839 & -5.779761 & -1.157173\end{array}$

$\begin{array}{llll}\text { C } & -1.098114 & -1.792362 & 6.259613\end{array}$

$\begin{array}{llll}\text { C } & -3.444587 & -3.556161 & 1.139614\end{array}$

$\begin{array}{llll}\mathrm{S} & 1.690477 & -5.054646 & 2.679016\end{array}$

$\begin{array}{llll}\text { C } & 0.845904 & -6.438812 & 3.532493\end{array}$

$\begin{array}{llll}\text { C } & 0.503249 & -3.934103 & -1.896769\end{array}$

$\begin{array}{llll}\text { C } & 1.192993 & -6.459091 & -0.709100\end{array}$

$\begin{array}{llll}\text { C } & 4.101003 & -4.343777 & 5.414358\end{array}$

$\begin{array}{llll}\text { C } & 3.744326 & -1.494660 & 5.456418\end{array}$

$\begin{array}{llll}\mathrm{H} & -0.431752 & -3.454837 & 3.134712\end{array}$

$\begin{array}{llll}\mathrm{H} & -3.868927 & -3.861111 & 0.171363\end{array}$

$\begin{array}{llll}\mathrm{H} & -4.167012 & -2.925436 & 1.674549\end{array}$

$\begin{array}{llll}\mathrm{H} & -3.220398 & -4.444756 & 1.746659\end{array}$

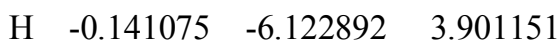

$\begin{array}{llll}\mathrm{H} & 1.479585 & -6.767501 & 4.369841\end{array}$

$\begin{array}{llll}\mathrm{H} & 0.738961 & -7.273487 & 2.823670\end{array}$

$\begin{array}{llll}\mathrm{H} & -2.102616 & -1.359862 & 6.159757\end{array}$

$\begin{array}{llll}\mathrm{H} & -0.645112 & -1.453611 & 7.203182\end{array}$

$\begin{array}{llll}\mathrm{H} & -1.172649 & -2.888989 & 6.247381\end{array}$

$\begin{array}{llll}\mathrm{H} & 2.357331 & 0.588486 & 1.635541\end{array}$

$\begin{array}{llll}\mathrm{H} & 1.996092 & -0.088984 & 3.257751\end{array}$

$\begin{array}{llll}\mathrm{H} & 2.179518 & -1.190074 & 1.857449\end{array}$

$\begin{array}{llll}\mathrm{H} & 0.222741 & 2.358811 & 1.799632\end{array}$

$\begin{array}{llll}\mathrm{H} & -1.469498 & 1.816152 & 2.079186\end{array}$

$\begin{array}{llll}\mathrm{H} & -0.283097 & 1.733348 & 3.410673\end{array}$

$\begin{array}{llll}\mathrm{H} & 0.635953 & 0.762109 & -0.361444\end{array}$

$\mathrm{H} \quad 0.315075 \quad-1.003875 \quad-0.394019$

$\begin{array}{llll}\mathrm{H} & -1.053675 & 0.144957 & -0.343106\end{array}$

$\begin{array}{llll}\mathrm{H} & 3.151478 & -3.123266 & 7.956824\end{array}$

$\mathrm{H} \quad 1.624244 \quad-2.293333 \quad 7.513061$

$\mathrm{H} \quad 1.713903 \quad-4.078714 \quad 7.459852$

$\mathrm{H} \quad 3.068091 \quad-0.651832 \quad 5.668753$

$\mathrm{H} \quad 4.561346 \quad-1.497096 \quad 6.196600$

H $\quad 4.171109 \quad-1.366031 \quad 4.449613$ 


$\begin{array}{crrr}\mathrm{H} & 3.649712 & -5.337184 & 5.561855 \\ \mathrm{H} & 4.557062 & -4.314451 & 4.413110 \\ \mathrm{H} & 4.880778 & -4.184543 & 6.177118 \\ \mathrm{H} & -3.841833 & -4.023714 & 4.902658 \\ \mathrm{H} & -1.418376 & -6.279329 & -2.129136 \\ \mathrm{H} & -1.953354 & -6.508558 & -0.429220 \\ \mathrm{H} & -2.304773 & -4.973710 & -1.276687 \\ \mathrm{H} & 1.189489 & -6.865591 & -1.733708 \\ \mathrm{H} & 2.204163 & -6.105863 & -0.456332 \\ \mathrm{H} & 0.919753 & -7.258850 & -0.003759 \\ \mathrm{H} & 0.562314 & -4.468016 & -2.859565 \\ \mathrm{H} & -0.240559 & -3.125786 & -1.975845 \\ \mathrm{H} & 1.485703 & -3.493306 & -1.667738\end{array}$

V

62

scf done: -3269.456871

$\begin{array}{llll}\mathrm{P} & -5.101386 & 1.711163 & 1.191699\end{array}$

$\begin{array}{cccc}\mathrm{Pd} & -4.015985 & 3.198511 & -0.184116\end{array}$

$\begin{array}{llll}\text { S } & -3.460697 & 4.187761 & -2.374918\end{array}$

$\begin{array}{llll}\text { C } & -5.013873 & 4.962078 & -2.929666\end{array}$

$\begin{array}{llll}\text { S } & -2.353379 & 3.845015 & 1.646447\end{array}$

$\begin{array}{llll}\mathrm{Pd} & -1.372768 & 5.861225 & 0.880242\end{array}$

$\begin{array}{llll}\mathrm{O} & -3.339457 & 6.793204 & 0.902344\end{array}$

$\begin{array}{llll}\text { C } & -3.976109 & 7.255356 & -0.067368\end{array}$

$\begin{array}{llll}\mathrm{O} & -3.685575 & 7.176063 & -1.281872\end{array}$

$\begin{array}{llll}\mathrm{Pd} & -2.020211 & 6.001831 & -1.905167\end{array}$

$\begin{array}{llll}\mathrm{P} & -0.396220 & 4.770929 & -2.904014\end{array}$

$\begin{array}{llll}\text { C } & -0.342028 & 3.018002 & -2.401561\end{array}$

$\begin{array}{llll}\text { C } & -3.421975 & 4.492194 & 2.976991\end{array}$

$\begin{array}{llll}\text { S } & -0.462420 & 7.453283 & -0.716653\end{array}$

$\begin{array}{llll}\text { C } & -1.316675 & 9.048460 & -0.470835\end{array}$

$\begin{array}{llll}\mathrm{P} & 0.619587 & 5.098511 & 1.627095\end{array}$

$\begin{array}{llll}\text { C } & 1.225609 & 3.510349 & 0.974846\end{array}$

$\begin{array}{llll}\text { C } & 1.318043 & 5.355806 & -2.699592\end{array}$

$\begin{array}{llll}\text { C } & -0.623030 & 4.711419 & -4.708237\end{array}$

$\begin{array}{llll}\text { C } & 2.019523 & 6.243828 & 1.414496\end{array}$

$\begin{array}{llll}\text { C } & 0.549219 & 4.833041 & 3.427822\end{array}$

$\begin{array}{llll}\mathrm{H} & -5.156700 & 2.661137 & -1.106320\end{array}$

$\begin{array}{llll}\mathrm{H} & -5.721989 & 4.153107 & -3.162770\end{array}$

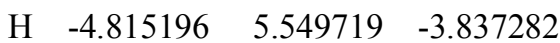

$\begin{array}{llll}\mathrm{H} & -5.433351 & 5.619212 & -2.157482\end{array}$

$\begin{array}{llll}\mathrm{H} & -4.140681 & 5.220447 & 2.576450\end{array}$

$\begin{array}{llll}\mathrm{H} & -2.795012 & 4.985882 & 3.735141\end{array}$

$\begin{array}{llll}\mathrm{H} & -3.953818 & 3.656978 & 3.459145\end{array}$

$\begin{array}{llll}\mathrm{H} & -2.055873 & 9.203558 & -1.268431\end{array}$

$\begin{array}{llll}\mathrm{H} & -0.561158 & 9.845808 & -0.504777\end{array}$

$\begin{array}{llll}\mathrm{H} & -1.826001 & 9.067219 & 0.502330\end{array}$

$\begin{array}{llll}\mathrm{H} & 2.001243 & 4.701868 & -3.265474\end{array}$

$\begin{array}{llll}\mathrm{H} & 1.405561 & 6.387637 & -3.071173\end{array}$

$\begin{array}{llll}\mathrm{H} & 1.607690 & 5.349529 & -1.637702\end{array}$

$\begin{array}{llll}\mathrm{H} & 0.165086 & 4.095247 & -5.171459\end{array}$

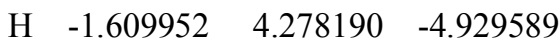

$\mathrm{H} \quad-0.581796 \quad 5.730292 \quad-5.121457$

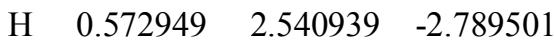

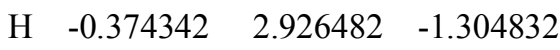

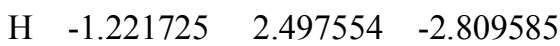

$\begin{array}{llll}\mathrm{H} & 2.930028 & 5.804458 & 1.853862\end{array}$ 


$\begin{array}{rrrr}\mathrm{H} & 2.185235 & 6.462133 & 0.349079 \\ \mathrm{H} & 1.790609 & 7.193545 & 1.921611 \\ \mathrm{H} & 1.477434 & 3.602990 & -0.092818 \\ \mathrm{H} & 2.129001 & 3.203826 & 1.527243 \\ \mathrm{H} & 0.446911 & 2.742097 & 1.099034 \\ \mathrm{H} & 0.206663 & 5.752875 & 3.926145 \\ \mathrm{H} & -0.160730 & 4.021336 & 3.647671 \\ \mathrm{H} & 1.549029 & 4.564053 & 3.807484 \\ \mathrm{H} & -4.911904 & 7.812447 & 0.174066 \\ \mathrm{C} & -4.070241 & 0.853276 & 2.432742 \\ \mathrm{C} & -6.485145 & 2.421849 & 2.146517 \\ \mathrm{C} & -5.888502 & 0.312359 & 0.322567 \\ \mathrm{H} & -6.944954 & 1.650206 & 2.786054 \\ \mathrm{H} & -6.139002 & 3.257799 & 2.772031 \\ \mathrm{H} & -7.240165 & 2.807966 & 1.444885 \\ \mathrm{H} & -4.688407 & 0.146167 & 3.009795 \\ \mathrm{H} & -3.271827 & 0.298584 & 1.916137 \\ \mathrm{H} & -3.597646 & 1.571074 & 3.118000 \\ \mathrm{H} & -6.347374 & -0.380733 & 1.046745 \\ \mathrm{H} & -6.660101 & 0.693400 & -0.363170 \\ \mathrm{H} & -5.134751 & -0.226678 & -0.271060\end{array}$

\section{VI}

74

scf done: -3347.480047

$\begin{array}{cccc}\mathrm{C} & 1.146784 & -1.492204 & -0.129481 \\ \mathrm{P} & 0.620130 & -0.898108 & 1.503193 \\ \mathrm{Pd} & 2.312192 & -0.083117 & 2.792779 \\ \mathrm{Pd} & 4.844070 & 0.001808 & 1.103197 \\ \mathrm{~S} & 5.138247 & 2.135833 & 0.080089 \\ \mathrm{C} & 6.595993 & 2.858026 & 0.895724 \\ \mathrm{C} & -0.775513 & 0.202571 & 1.121479 \\ \mathrm{C} & -0.128618 & -2.345593 & 2.313410 \\ \mathrm{O} & 3.638224 & 0.456949 & 4.373509 \\ \mathrm{C} & 4.833816 & 0.776710 & 4.181281 \\ \mathrm{O} & 5.452117 & 0.817769 & 3.099161 \\ \mathrm{~S} & 1.251587 & 2.069172 & 2.837964 \\ \mathrm{C} & 1.563577 & 2.751926 & 4.497170 \\ \mathrm{Pd} & 3.208801 & 2.576475 & 1.488711 \\ \mathrm{C} & 3.530665 & 4.746938 & 2.417827 \\ \mathrm{~S} & 3.745865 & -1.922483 & 2.093075 \\ \mathrm{C} & 4.755141 & -2.416206 & 3.531060 \\ \mathrm{P} & 5.425833 & -1.144126 & -0.764084 \\ \mathrm{C} & 6.093874 & -0.182426 & -2.157705 \\ \mathrm{C} & 6.802868 & -2.238311 & -0.283123 \\ \mathrm{C} & 4.238775 & -2.277970 & -1.551389 \\ \mathrm{H} & 2.640349 & 1.394314 & 0.633269 \\ \mathrm{H} & 5.170135 & -3.413828 & 3.326525 \\ \mathrm{H} & 4.119428 & -2.460799 & 4.426381 \\ \mathrm{H} & 5.576095 & -1.706428 & 3.698965 \\ \mathrm{H} & 6.706377 & 2.469859 & 1.918131 \\ \mathrm{H} & 6.485713 & 3.952924 & 0.910348 \\ \mathrm{H} & 7.486974 & 2.597563 & 0.304644 \\ \mathrm{H} & 2.504541 & 2.366869 & 4.912980 \\ \mathrm{H} & 0.728207 & 2.456189 & 5.147908 \\ \mathrm{H} & 1.596700 & 3.849716 & 4.434059 \\ \mathrm{H} & -1.495144 & -0.341003 & 0.486533 \\ \mathrm{H} & -1.266713 & 0.536819 & 2.046577\end{array}$




\begin{tabular}{rrrr}
$\mathrm{H}$ & -0.407739 & 1.087010 & 0.576025 \\
$\mathrm{H}$ & -0.938503 & -2.755578 & 1.687854 \\
$\mathrm{H}$ & 0.644457 & -3.113599 & 2.467007 \\
$\mathrm{H}$ & -0.536084 & -2.053544 & 3.292882 \\
$\mathrm{H}$ & 0.280976 & -1.884635 & -0.687997 \\
$\mathrm{H}$ & 1.595559 & -0.655334 & -0.690853 \\
$\mathrm{H}$ & 1.892468 & -2.289906 & -0.002564 \\
$\mathrm{H}$ & 5.406593 & 1.063045 & 5.097167 \\
$\mathrm{H}$ & 7.164071 & -2.801313 & -1.160163 \\
$\mathrm{H}$ & 7.627714 & -1.639153 & 0.131338 \\
$\mathrm{H}$ & 6.459093 & -2.945221 & 0.487508 \\
$\mathrm{H}$ & 6.440927 & -0.873890 & -2.943052 \\
$\mathrm{H}$ & 5.322371 & 0.487669 & -2.567353 \\
$\mathrm{H}$ & 6.936288 & 0.435891 & -1.813107 \\
$\mathrm{H}$ & 4.752461 & -2.821661 & -2.361808 \\
$\mathrm{H}$ & 3.869813 & -3.002054 & -0.808881 \\
$\mathrm{H}$ & 3.388908 & -1.719175 & -1.974022 \\
$\mathrm{C}$ & 4.379630 & 4.026777 & 2.928269 \\
$\mathrm{C}$ & 2.589372 & 5.765228 & 1.938818 \\
$\mathrm{O}$ & 2.884541 & 6.228727 & 0.656059 \\
$\mathrm{C}$ & 1.982250 & 5.875619 & -0.369208 \\
$\mathrm{C}$ & 2.171620 & 4.506236 & -0.929286 \\
$\mathrm{C}$ & 1.144191 & 3.681101 & -1.187255 \\
$\mathrm{C}$ & 1.179143 & 2.402572 & -1.900457 \\
$\mathrm{C}$ & -0.029490 & 1.726295 & -2.131129 \\
$\mathrm{H}$ & 5.186036 & 3.531783 & 3.445585 \\
$\mathrm{C}$ & -0.068749 & 0.536544 & -2.853875 \\
$\mathrm{C}$ & 1.107354 & -0.004359 & -3.367386 \\
$\mathrm{C}$ & 2.319412 & 0.653204 & -3.143081 \\
$\mathrm{C}$ & 2.360080 & 1.837845 & -2.415318 \\
$\mathrm{H}$ & 2.155459 & 6.617940 & -1.169162 \\
$\mathrm{H}$ & 0.933422 & 6.011517 & -0.034187 \\
$\mathrm{H}$ & 1.555470 & 5.368408 & 2.007245 \\
$\mathrm{H}$ & 2.648454 & 6.613985 & 2.646858 \\
$\mathrm{H}$ & 3.199036 & 4.244321 & -1.219692 \\
$\mathrm{H}$ & 0.140212 & 4.004668 & -0.872602 \\
$\mathrm{H}$ & -0.960082 & 2.161971 & -1.749852 \\
$\mathrm{H}$ & -1.026353 & 0.037067 & -3.029163 \\
$\mathrm{H}$ & 1.082702 & -0.928938 & -3.951863 \\
$\mathrm{H}$ & 3.243137 & 0.244801 & -3.567563 \\
$\mathrm{H}$ & 3.317698 & 2.348990 & -2.265457 \\
& & & \\
\hline
\end{tabular}

\begin{tabular}{|c|c|c|c|}
\hline \multirow{2}{*}{\multicolumn{4}{|c|}{ VII }} \\
\hline & & \\
\hline \multicolumn{4}{|c|}{$\begin{array}{l}74 \\
\text { scf done: }-3347.479326\end{array}$} \\
\hline $\mathrm{C}$ & 0.348702 & 0.125805 & -0.142383 \\
\hline $\mathrm{C}$ & 0.147132 & 0.015197 & 1.229134 \\
\hline $\mathrm{C}$ & 1.239498 & -0.005291 & 2.112479 \\
\hline $\mathrm{C}$ & 2.532489 & 0.093703 & 1.576816 \\
\hline $\mathrm{C}$ & 2.733370 & 0.204538 & 0.203082 \\
\hline $\mathrm{C}$ & 1.641473 & 0.222068 & -0.662120 \\
\hline $\mathrm{C}$ & 1.086234 & -0.120379 & 3.568935 \\
\hline $\mathrm{Pd}$ & 0.183811 & 1.998535 & 4.197766 \\
\hline $\mathrm{S}$ & -2.229553 & 2.319509 & 3.939931 \\
\hline $\mathrm{C}$ & -2.904085 & 2.492305 & 5.620622 \\
\hline $\mathrm{C}$ & -0.064260 & -0.372947 & 4.259040 \\
\hline $\mathrm{C}$ & -0.057198 & -0.705411 & 5.712898 \\
\hline $\mathrm{O}$ & -1.248822 & -0.256034 & 6.305350 \\
\hline
\end{tabular}




\begin{tabular}{|c|c|c|c|}
\hline $\mathrm{C}$ & -1.178492 & -0.132930 & .700877 \\
\hline $\mathrm{C}$ & -0.425952 & 1.047569 & 8.128063 \\
\hline $\mathrm{C}$ & 0.184293 & 2.035449 & 474519 \\
\hline $\mathrm{S}$ & 2.495260 & 2.816380 & .317588 \\
\hline $\mathrm{Pd}$ & 1.457532 & 4.854630 & 3.573295 \\
\hline $\mathrm{O}$ & 0.908401 & 5.470215 & 5.593495 \\
\hline $\mathrm{C}$ & -0.259048 & 5.504425 & 6.048790 \\
\hline $\mathrm{O}$ & -1.324380 & 5.217785 & 5.463645 \\
\hline $\mathrm{Pd}$ & -1.567799 & 4.542799 & 3.360887 \\
\hline$P$ & -2.690136 & 4.247413 & 1.408133 \\
\hline $\mathrm{C}$ & -2.489119 & 5.543409 & 0.145142 \\
\hline $\mathrm{C}$ & 2.785972 & 3.017143 & 6.105580 \\
\hline $\mathrm{S}$ & -0.164917 & 8317 & 2.572255 \\
\hline $\mathrm{C}$ & -0.353500 & 7.771610 & 3.696981 \\
\hline $\mathrm{P}$ & 2.422201 & 4.606953 & 1.520471 \\
\hline $\mathrm{C}$ & 3.966022 & 441 & 1.440599 \\
\hline $\mathrm{C}$ & 2.861491 & 49 & 0.749017 \\
\hline $\mathrm{C}$ & 1.32 & & 0.318757 \\
\hline $\mathrm{C}$ & -2.385380 & 226 & 0.506544 \\
\hline $\mathrm{C}$ & -4.479660 & 980 & 1.743300 \\
\hline $\mathrm{H}$ & -0.7 & 707 & 4702 \\
\hline $\mathrm{H}$ & 0.624052 & 348 & 4.124748 \\
\hline $\mathrm{H}$ & -1.061877 & 472 & 4.502773 \\
\hline $\mathrm{H}$ & -2.287093 & 557 & 6.221018 \\
\hline $\mathrm{H}$ & -2.913132 & 443 & 6.0 \\
\hline $\mathrm{H}$ & -3.9 & 99 & 328 \\
\hline $\mathrm{H}$ & 2.147791 & 89 & 6.505083 \\
\hline $\mathrm{H}$ & 3.844509 & 105 & 6.258422 \\
\hline $\mathrm{H}$ & 2.5 & 93 & 6.611902 \\
\hline $\mathrm{H}$ & 4.322186 & 296 & 0.397103 \\
\hline $\mathrm{H}$ & 4.72 & 64 & 2.08 \\
\hline $\mathrm{H}$ & 3.794487 & 500 & 1.795956 \\
\hline $\mathrm{H}$ & 3.320216 & 391 & -0.237372 \\
\hline $\mathrm{H}$ & 1.9 & 79 & 0.63 \\
\hline $\mathrm{H}$ & 3.578860 & 040 & 1.391015 \\
\hline $\mathrm{H}$ & 1.845178 & 78 & -0.650034 \\
\hline $\mathrm{H}$ & 1.0 & 48 & 0.696850 \\
\hline $\mathrm{H}$ & 0.418662 & 4.3 & 0.174975 \\
\hline $\mathrm{H}$ & -0.3 & 332 & 7.110587 \\
\hline $\mathrm{H}$ & -3.137245 & 5.316178 & -0.717230 \\
\hline $\mathrm{H}$ & -2.769437 & 71 & 0.568161 \\
\hline $\mathrm{H}$ & -1.443502 & & -0.191289 \\
\hline $\mathrm{H}$ & -5.042260 & 219 & 0.799904 \\
\hline $\mathrm{H}$ & -4.716209 & 123 & 2.403586 \\
\hline $\mathrm{H}$ & -4.769816 & 5.165678 & 2.250015 \\
\hline $\mathrm{H}$ & -3.041338 & 2.646499 & -0.378313 \\
\hline $\mathrm{H}$ & -1.333746 & 142 & 0.186346 \\
\hline $\mathrm{H}$ & -2.597606 & 1.838485 & 1.164963 \\
\hline $\mathrm{H}$ & 0.721134 & 2.906238 & 8.806811 \\
\hline $\mathrm{H}$ & 0.038032 & -1.805130 & 5.853160 \\
\hline $\mathrm{H}$ & 0.830358 & -0.241679 & 6.194123 \\
\hline $\mathrm{H}$ & -0.741541 & -1.043342 & 8.160963 \\
\hline $\mathrm{H}$ & -2.214536 & -0.062427 & 8.073331 \\
\hline $\mathrm{H}$ & -1.013229 & -0.551246 & 3.736634 \\
\hline $\mathrm{H}$ & 2.029007 & -0.125216 & 4.134128 \\
\hline $\mathrm{H}$ & 3.392747 & 0.069979 & 2.255392 \\
\hline $\mathrm{H}$ & 3.750793 & 0.270551 & -0.193990 \\
\hline $\mathrm{H}$ & 1.794185 & 0.299647 & -1.742173 \\
\hline $\mathrm{H}$ & -0.513103 & 0.126895 & -0.816945 \\
\hline- & -0.872560 & -0.064946 & 1.62201 \\
\hline
\end{tabular}


H $\quad 0.005366 \quad 3.526284 \quad 4.399373$

TS (VII-VIII)

74

scf done: -3347.465513

$\begin{array}{llll}\text { C } & -0.006761 & 0.037842 & 0.070670\end{array}$

$\begin{array}{llll}\text { C } & 0.011257 & 0.032229 & 1.463399\end{array}$

$\begin{array}{llll}\text { C } & 1.234524 & -0.021415 & 2.134641\end{array}$

$\begin{array}{llll}\text { C } & 2.426894 & -0.059334 & 1.421770\end{array}$

$\begin{array}{llll}\text { C } & 2.426255 & -0.040885 & 0.017199\end{array}$

$\begin{array}{llll}\text { C } & 1.189106 & 0.001483 & -0.642085\end{array}$

$\begin{array}{llll}\text { C } & 3.658845 & -0.061084 & -0.775916\end{array}$

$\begin{array}{llll}\text { C } & 4.881201 & -0.403110 & -0.345348\end{array}$

$\begin{array}{llll}\text { C } & 6.095151 & -0.435379 & -1.210539\end{array}$

$\begin{array}{llll}\text { O } & 7.208247 & 0.229495 & -0.648265\end{array}$

$\begin{array}{llll}\text { C } & 7.122824 & 1.619058 & -0.632364\end{array}$

$\begin{array}{llll}\text { C } & 6.344451 & 2.133593 & 0.525674\end{array}$

$\begin{array}{llll}\mathrm{Pd} & 4.456474 & 3.187759 & 0.592991\end{array}$

$\begin{array}{llll}\mathrm{S} & 2.081020 & 3.588198 & 1.048927\end{array}$

$\begin{array}{llll}\text { C } & 1.905327 & 3.901470 & 2.833795\end{array}$

$\begin{array}{llll}\text { C } & 6.029122 & 2.219301 & 1.736059\end{array}$

$\begin{array}{llll}\mathrm{S} & 5.158838 & 4.276932 & -1.605714\end{array}$

C $\quad 6.735530 \quad 5.033531 \quad-1.067992$

$\begin{array}{llll}\mathrm{Pd} & 3.799077 & 6.227309 & -1.660078\end{array}$

$\begin{array}{llll}\text { O } & 4.560128 & 6.789122 & 0.309692\end{array}$

$\begin{array}{llll}\text { C } & 3.935443 & 6.927825 & 1.380769\end{array}$

$\begin{array}{llll}\text { O } & 2.734339 & 6.650519 & 1.605837\end{array}$

$\begin{array}{llll}\mathrm{Pd} & 1.600192 & 5.690442 & 0.081021\end{array}$

$\begin{array}{llll}\mathrm{P} & 0.195465 & 4.550013 & -1.288942\end{array}$

C $\quad-0.613791 \quad 5.530320 \quad-2.592995$

S $\quad 1.820498 \quad 7.598656 \quad-1.425871$

$\begin{array}{llll}\text { C } & 2.267531 & 9.051128 & -0.413312\end{array}$

$\begin{array}{llll}\text { P } & 3.796624 & 6.142508 & -3.923272\end{array}$

$\begin{array}{llll}\text { C } & 2.890074 & 7.484601 & -4.754734\end{array}$

C $\quad 0.995175 \quad 3.169500 \quad-2.164091$

$\begin{array}{llll}\text { C } & -1.186929 & 3.783721 & -0.386760\end{array}$

$\begin{array}{llll}\text { C } & 5.509292 & 6.358953 & -4.504372\end{array}$

C $\quad 3.241391 \quad 4.632940 \quad-4.777893$

$\begin{array}{llll}\mathrm{H} & 4.262689 & 2.587423 & 2.019301\end{array}$

$\begin{array}{llll}\mathrm{H} & 2.175310 & 2.978479 & 3.370189\end{array}$

$\mathrm{H} \quad 0.854055 \quad 4.147816 \quad 3.043648$

H $\quad 2.548074 \quad 4.731912 \quad 3.153159$

$\begin{array}{llll}\mathrm{H} & 6.617430 & 5.507417 & -0.084037\end{array}$

$\mathrm{H} \quad 7.037488 \quad 5.795007 \quad-1.802585$

$\mathrm{H} \quad 7.519875 \quad 4.264061 \quad-1.024722$

$\begin{array}{llll}\mathrm{H} & 2.127839 & 8.836330 & 0.654582\end{array}$

$\begin{array}{llll}\mathrm{H} & 1.613294 & 9.882595 & -0.711043\end{array}$

$\mathrm{H} \quad 3.315008 \quad 9.327297 \quad-0.596885$

H $\quad-1.256785 \quad 4.878625 \quad-3.206787$

$\mathrm{H} \quad-1.226610 \quad 6.320353 \quad-2.132835$

$\mathrm{H} \quad 0.138267 \quad 6.015367 \quad-3.232987$

H $\quad-1.859799 \quad 3.271034 \quad-1.094139$

$\mathrm{H} \quad-0.787108 \quad 3.054273 \quad 0.334573$

$\begin{array}{llll}\mathrm{H} & -1.751239 & 4.556584 & 0.156661\end{array}$

$\mathrm{H} \quad \begin{array}{llll}1.852500 & 3.542407 & -2.743587\end{array}$

$\mathrm{H} \quad 1.362226 \quad 2.435145 \quad-1.428789$

H $\quad 0.275322 \quad 2.674799 \quad-2.838112$

H $3.048643 \quad 7.414562 \quad-5.843447$ 


$\begin{array}{rrrr}\mathrm{H} & 1.814326 & 7.425717 & -4.534319 \\ \mathrm{H} & 3.261807 & 8.455484 & -4.393370 \\ \mathrm{H} & 2.149917 & 4.520915 & -4.690901 \\ \mathrm{H} & 3.502355 & 4.704837 & -5.846714 \\ \mathrm{H} & 3.738218 & 3.752502 & -4.342103 \\ \mathrm{H} & 5.942828 & 7.268891 & -4.061906 \\ \mathrm{H} & 6.109605 & 5.488574 & -4.199458 \\ \mathrm{H} & 5.520896 & 6.446668 & -5.603285 \\ \mathrm{H} & 4.505784 & 7.355531 & 2.238290 \\ \mathrm{H} & 6.154565 & 2.094330 & 2.803665 \\ \mathrm{H} & 6.704424 & 2.008361 & -1.583530 \\ \mathrm{H} & 8.153592 & 2.006925 & -0.542890 \\ \mathrm{H} & 6.430675 & -1.477261 & -1.354238 \\ \mathrm{H} & 5.862779 & -0.021907 & -2.214649 \\ \mathrm{H} & 5.039031 & -0.715926 & 0.695836 \\ \mathrm{H} & 3.542803 & 0.220673 & -1.834658 \\ \mathrm{H} & 1.172617 & -0.005106 & -1.737923 \\ \mathrm{H} & -0.960431 & 0.062981 & -0.466262 \\ \mathrm{H} & -0.924970 & 0.057883 & 2.028197 \\ \mathrm{H} & 1.256326 & -0.035607 & 3.228277 \\ \mathrm{H} & 3.377909 & -0.096370 & 1.963772\end{array}$

\begin{tabular}{cccc}
\multicolumn{4}{c}{ VIII } \\
74 & & \\
scf done: -3347.519802 \\
C & -1.735682 & -0.494038 & 1.025992 \\
C & -0.905308 & -0.054882 & 2.049633 \\
C & 0.443865 & 0.243665 & 1.796501 \\
C & 0.932478 & 0.089653 & 0.491820 \\
C & 0.100898 & -0.355688 & -0.532488 \\
C & -1.235631 & -0.645311 & -0.268980 \\
C & 1.354254 & 0.710808 & 2.851534 \\
C & 1.134204 & 0.547339 & 4.200653 \\
C & 2.193839 & 0.589770 & 5.260916 \\
O & 1.664086 & 0.858207 & 6.524655 \\
C & 0.994971 & 2.092148 & 6.639024 \\
C & -0.049623 & 2.285885 & 5.583389 \\
Pd & 0.687392 & 2.775393 & 3.728164 \\
S & 1.303445 & 3.352983 & 1.268329 \\
C & -0.419916 & 3.499501 & 0.703501 \\
C & -1.358435 & 2.132899 & 5.777894 \\
S & 0.267162 & 4.951925 & 4.793439 \\
C & -1.496873 & 5.311881 & 4.560125 \\
Pd & 1.422318 & 6.689599 & 3.638959 \\
P & 3.044581 & 6.506645 & 5.215509 \\
C & 3.859902 & 4.877166 & 5.254574 \\
O & -0.320405 & 7.170953 & 2.499470 \\
C & -0.473587 & 6.925833 & 1.283385 \\
O & 0.313736 & 6.325985 & 0.520215 \\
Pd & 2.191962 & 5.540674 & 1.145696 \\
S & 2.913803 & 7.710759 & 1.992265 \\
C & 1.970029 & 9.005426 & 1.115908 \\
P & 4.277289 & 4.634876 & 1.119255 \\
C & 4.687454 & 4.334996 & -0.629411 \\
C & 5.662502 & 5.653754 & 1.723196 \\
C & 4.536094 & 3.006554 & 1.898951 \\
C & 4.402066 & 7.713162 & 5.108151 \\
C & 2.392914 & 6.737266 & 6.898363 \\
& & &
\end{tabular}




$\begin{array}{rrrr}\mathrm{H} & -2.096902 & 2.211530 & 4.969966 \\ \mathrm{H} & -0.853879 & 2.486761 & 0.725381 \\ \mathrm{H} & -0.450951 & 3.890620 & -0.322594 \\ \mathrm{H} & -0.999280 & 4.163372 & 1.362433 \\ \mathrm{H} & -1.772660 & 5.365163 & 3.498992 \\ \mathrm{H} & -1.715981 & 6.278319 & 5.035894 \\ \mathrm{H} & -2.061059 & 4.517345 & 5.070000 \\ \mathrm{H} & 1.588134 & 8.619818 & 0.160573 \\ \mathrm{H} & 2.649979 & 9.848096 & 0.927969 \\ \mathrm{H} & 1.127371 & 9.344208 & 1.733810 \\ \mathrm{H} & 6.613940 & 5.143659 & 1.499823 \\ \mathrm{H} & 5.642298 & 6.635060 & 1.226330 \\ \mathrm{H} & 5.591630 & 5.816811 & 2.809746 \\ \mathrm{H} & 5.696905 & 3.900240 & -0.717902 \\ \mathrm{H} & 3.947208 & 3.642365 & -1.058372 \\ \mathrm{H} & 4.646497 & 5.282010 & -1.188338 \\ \mathrm{H} & 5.597729 & 2.722352 & 1.818795 \\ \mathrm{H} & 4.238339 & 3.015624 & 2.958993 \\ \mathrm{H} & 3.921635 & 2.262641 & 1.368328 \\ \mathrm{H} & 5.125262 & 7.536318 & 5.920884 \\ \mathrm{H} & 4.906012 & 7.648194 & 4.132650 \\ \mathrm{H} & 3.985912 & 8.727672 & 5.204615 \\ \mathrm{H} & 4.542269 & 4.789535 & 4.395523 \\ \mathrm{H} & 4.439020 & 4.751870 & 6.183891 \\ \mathrm{H} & 3.099339 & 4.082224 & 5.179856 \\ \mathrm{H} & 1.900923 & 7.719086 & 6.972114 \\ \mathrm{H} & 1.650825 & 5.954719 & 7.115964 \\ \mathrm{H} & 3.218145 & 6.686699 & 7.627375 \\ \mathrm{H} & -1.421694 & 7.289824 & 0.823983 \\ \mathrm{H} & -1.751041 & 1.914516 & 6.781992 \\ \mathrm{H} & 1.724809 & 2.930847 & 6.618144 \\ \mathrm{H} & 0.524394 & 2.093365 & 7.637958 \\ \mathrm{H} & 2.658845 & -0.409845 & 5.335674 \\ \mathrm{H} & 3.002880 & 1.301146 & 4.980148 \\ \mathrm{H} & 0.218536 & 0.036307 & 4.519364 \\ \mathrm{H} & 2.371951 & 0.961584 & 2.522235 \\ \mathrm{H} & 1.982043 & 0.321653 & 0.283622 \\ \mathrm{H} & 0.499931 & -0.475708 & -1.543425 \\ \mathrm{H} & -1.891597 & -0.991643 & -1.072262 \\ \mathrm{H} & -2.784462 & -0.719806 & 1.237601 \\ \mathrm{H} & -1.314239 & 0.066966 & 3.058629\end{array}$

\section{TS (VIII-IX)}

74

scf done: -3347.513598

$\begin{array}{lrrr}\mathrm{C} & 1.219386 & -0.030867 & -0.810186 \\ \mathrm{C} & -0.005263 & -0.061998 & -0.125571 \\ \mathrm{C} & -0.043286 & -0.079029 & 1.265622 \\ \mathrm{C} & 1.141555 & -0.058421 & 1.998618 \\ \mathrm{C} & 2.367115 & -0.028936 & 1.330438 \\ \mathrm{C} & 2.406492 & -0.017034 & -0.058717 \\ \mathrm{C} & 1.224607 & 0.000901 & -2.278913 \\ \mathrm{C} & 2.319944 & -0.451571 & -3.059559 \\ \mathrm{C} & 2.093479 & -1.033979 & -4.429944 \\ \mathrm{O} & 3.242864 & -0.926845 & -5.214170 \\ \mathrm{C} & 3.748834 & 0.384831 & -5.170749 \\ \mathrm{C} & 3.718617 & 0.889922 & -3.761261 \\ \mathrm{C} & 4.803429 & 1.135008 & -3.019037\end{array}$




\begin{tabular}{|c|c|c|c|}
\hline $\mathrm{Pd}$ & 796 & 624 & \\
\hline S & -0.069430 & 2.808519 & -1.945261 \\
\hline $\mathrm{C}$ & 0.765712 & 3.472672 & -0.470793 \\
\hline S & 3.012032 & 3.735419 & -4.403503 \\
\hline $\mathrm{C}$ & 4.098935 & 4.521313 & -3.176213 \\
\hline $\mathrm{Pd}$ & 1.359137 & 5.348502 & -4.930741 \\
\hline $\mathrm{O}$ & 1.931733 & 6.577451 & -3.288866 \\
\hline $\mathrm{C}$ & 1.306295 & 6.639798 & -2.208459 \\
\hline $\mathrm{O}$ & 0.265767 & 6.024541 & -1.890092 \\
\hline $\mathrm{Pd}$ & -0.805064 & 4.695729 & -3.174975 \\
\hline $\mathbf{r}$ & -2.442466 & 3.393808 & -4.066664 \\
\hline $\mathrm{C}$ & -2.144141 & 1.601096 & -4.230711 \\
\hline $\mathrm{P}$ & 1.140199 & 4.328938 & 5196 \\
\hline $\mathrm{C}$ & 2.717246 & 4.227648 & -7.846833 \\
\hline S & -0.874489 & 2766 & 6358 \\
\hline $\mathrm{C}$ & -0.741278 & 8.007866 & -4.238546 \\
\hline $\mathrm{C}$ & 0.530 & 2.61 & -6.868664 \\
\hline $\mathrm{C}$ & 0.023745 & 905 & 1500 \\
\hline $\mathrm{C}$ & -3.862576 & 0072 & -2.5 \\
\hline $\mathrm{C}$ & -3.1 & 834 & 676 \\
\hline $\mathrm{H}$ & 4.742747 & 1.418348 & -1.9 \\
\hline $\mathrm{H}$ & 1.112862 & 175 & \\
\hline $\mathrm{H}$ & 0.0 & 36 & 11 \\
\hline $\mathrm{H}$ & 1.620032 & 4.108312 & -0.740622 \\
\hline $\mathrm{H}$ & 3.55 & 565 & -2.259267 \\
\hline $\mathrm{H}$ & 4.547950 & 5.425203 & 404 \\
\hline $\mathrm{H}$ & 4.89 & 770 & -2.9 \\
\hline $\mathrm{H}$ & -1.1 & 695 & -3 \\
\hline $\mathrm{H}$ & -1.355904 & 8.692678 & -4.839428 \\
\hline $\mathrm{H}$ & 0.304123 & 8.345216 & -4.246122 \\
\hline $\mathrm{H}$ & -4.02 & 623 & -5.8 \\
\hline $\mathrm{H}$ & -3.425183 & 4.943721 & -5.6 \\
\hline $\mathrm{H}$ & -2.4 & 617 & 312 \\
\hline $\mathrm{H}$ & -4.687184 & 2.848209 & 9757 \\
\hline $\mathrm{H}$ & -3.54 & 3.130461 & -1.9 \\
\hline $\mathrm{H}$ & -4.20 & 739 & 4760 \\
\hline $\mathrm{H}$ & -3.018307 & 1.126943 & -4.706154 \\
\hline $\mathrm{H}$ & -1.245306 & 1.398539 & -4.8 \\
\hline $\mathrm{H}$ & -1.999491 & 1.167008 & 9173 \\
\hline $\mathrm{H}$ & 0.017712 & 4.607702 & -9.077740 \\
\hline $\mathrm{H}$ & -0.99 & 808 & 3574 \\
\hline $\mathrm{H}$ & 0.377038 & 6.184513 & -8.291383 \\
\hline $\mathrm{H}$ & -0.534968 & 2.626118 & -6.5 \\
\hline $\mathrm{H}$ & 0.6 & 2.112 & 4788 \\
\hline $\mathrm{H}$ & 1.081791 & 2.050421 & -6.096460 \\
\hline $\mathrm{H}$ & 3.135892 & 5.238811 & -7.9 \\
\hline $\mathrm{H}$ & 3.433140 & 3.619153 & -7.2 \\
\hline $\mathrm{H}$ & 2.551873 & 3.782120 & -8.8 \\
\hline $\mathrm{H}$ & 1.721050 & 7.333192 & -1.440389 \\
\hline $\mathrm{H}$ & 5.809321 & 1.069175 & -3.456056 \\
\hline $\mathrm{H}$ & 3.158599 & 1.056315 & -5.8 \\
\hline $\mathrm{H}$ & 4.781422 & 0.357116 & -5.557438 \\
\hline $\mathrm{H}$ & 1.860741 & -2.108363 & -4.336951 \\
\hline $\mathrm{H}$ & 1.215700 & -0.541445 & -4.905713 \\
\hline $\mathrm{H}$ & 3.135738 & -0.932443 & -2.509890 \\
\hline $\mathrm{H}$ & 0.230454 & -0.052224 & -2.748233 \\
\hline $\mathrm{H}$ & -0.936333 & -0.076650 & -0.701648 \\
\hline $\mathrm{H}$ & -1.006476 & -0.110393 & 1.782445 \\
\hline $\mathrm{H}$ & 1.112199 & -0.070208 & 3.091338 \\
\hline $\mathrm{H}$ & 3.300357 & -0.016887 & 1.900098 \\
\hline
\end{tabular}


IX

74

scf done: -3347.554382

$\begin{array}{llll}\text { C } & -0.046953 & -0.104206 & 0.336869\end{array}$

$\begin{array}{llll}\text { C } & -0.041242 & -0.199841 & 1.728394\end{array}$

$\begin{array}{llll}\text { C } & 1.155144 & -0.115226 & 2.432915\end{array}$

$\begin{array}{llll}\text { C } & 2.377655 & 0.080215 & 1.766145\end{array}$

$\begin{array}{llll}\text { C } & 2.353620 & 0.158670 & 0.361544\end{array}$

$\begin{array}{llll}\text { C } & 1.158138 & 0.064797 & -0.343624\end{array}$

$\begin{array}{llll}\text { C } & 3.658365 & 0.202601 & 2.483157\end{array}$

$\begin{array}{llll}\text { Pd } & 3.867186 & 2.122338 & 3.394464\end{array}$

$\begin{array}{llll}\mathrm{S} & 4.138800 & 4.134346 & 5.001534\end{array}$

$\begin{array}{llll}\mathrm{Pd} & 5.253720 & 5.878468 & 3.846870\end{array}$

$\begin{array}{llll}\text { S } & 6.575660 & 6.863587 & 2.045446\end{array}$

$\begin{array}{llll}\mathrm{C} & 5.744030 & 8.384738 & 1.473086\end{array}$

$\begin{array}{llll}\text { C } & 3.902279 & -0.631110 & 3.723027\end{array}$

$\begin{array}{llll}\text { C } & 5.281451 & -1.305634 & 3.793462\end{array}$

$\begin{array}{llll}\text { O } & 5.711275 & -1.159724 & 5.124445\end{array}$

$\begin{array}{llll}\text { C } & 5.358466 & 0.138996 & 5.514232\end{array}$

$\begin{array}{llll}\text { C } & 4.017950 & 0.366674 & 4.862008\end{array}$

$\begin{array}{llll}\text { C } & 3.028760 & 1.203856 & 5.292262\end{array}$

$\begin{array}{llll}\text { S } & 4.047653 & 2.974032 & 1.103672\end{array}$

$\begin{array}{llll}\mathrm{Pd} & 5.345342 & 4.963647 & 1.145000\end{array}$

$\begin{array}{llll}\mathrm{P} & 7.199111 & 3.680931 & 0.850614\end{array}$

$\begin{array}{llll}\text { C } & 7.224580 & 2.048304 & 1.662843\end{array}$

$\begin{array}{llll}\text { C } & 2.325781 & 3.550651 & 0.930428\end{array}$

$\begin{array}{llll}\text { C } & 2.418648 & 4.707155 & 5.182069\end{array}$

$\begin{array}{llll}\mathrm{P} & 7.024346 & 5.511111 & 5.208880\end{array}$

$\begin{array}{llll}\text { C } & 6.590346 & 5.919616 & 6.927742\end{array}$

$\begin{array}{llll}\text { O } & 3.390352 & 6.635593 & 3.075506\end{array}$

$\begin{array}{llll}\text { C } & 3.046366 & 6.695401 & 1.877122\end{array}$

$\begin{array}{lllll}\text { O } & 3.623185 & 6.179961 & 0.893264\end{array}$

$\begin{array}{llll}\text { C } & 7.640081 & 3.798469 & 5.292674\end{array}$

$\begin{array}{llll}\text { C } & 8.510298 & 6.509505 & 4.882179\end{array}$

$\begin{array}{llll}\text { C } & 7.356303 & 3.291822 & -0.920725\end{array}$

$\begin{array}{llll}\text { C } & 8.300754 & 4.421917 & 1.299319\end{array}$

$\mathrm{H} \quad 1.999846 \quad 1.127785 \quad 4.920606$

$\begin{array}{llll}\mathrm{H} & 1.683700 & 2.655749 & 0.893456\end{array}$

$\begin{array}{llll}\mathrm{H} & 2.224158 & 4.120677 & -0.003071\end{array}$

$\begin{array}{llll}\mathrm{H} & 2.032217 & 4.185022 & 1.779415\end{array}$

$\begin{array}{llll}\mathrm{H} & 1.966783 & 4.924301 & 4.204951\end{array}$

$\begin{array}{llll}\mathrm{H} & 2.393619 & 5.617819 & 5.797150\end{array}$

$\begin{array}{llll}\mathrm{H} & 1.846059 & 3.916588 & 5.690588\end{array}$

$\begin{array}{llll}\mathrm{H} & 5.108251 & 8.167762 & 0.604394\end{array}$

$\begin{array}{llll}\mathrm{H} & 6.522582 & 9.107629 & 1.191954\end{array}$

$\begin{array}{llll}\mathrm{H} & 5.127560 & 8.808218 & 2.278124\end{array}$

$\begin{array}{llll}\mathrm{H} & 9.619076 & 3.746348 & 1.002454\end{array}$

$\begin{array}{llll}\mathrm{H} & 8.919265 & 5.393302 & 0.797016\end{array}$

$\begin{array}{llll}\mathrm{H} & 8.850308 & 4.590069 & 2.386202\end{array}$

$\begin{array}{llll}\mathrm{H} & 8.251226 & 2.673582 & -1.101740\end{array}$

H $\quad 6.458012 \quad 2.746394 \quad-1.248546$

$\begin{array}{llll}\mathrm{H} & 7.430365 & 4.223824 & -1.500907\end{array}$

$\mathrm{H} \quad 8.197576 \quad 1.558719 \quad 1.493395$

$\begin{array}{llll}\mathrm{H} & 7.041978 & 2.152020 & 2.744761\end{array}$

$\begin{array}{llll}\mathrm{H} & 6.427065 & 1.418651 & 1.237905\end{array}$

$\begin{array}{llll}\mathrm{H} & 9.287621 & 6.271507 & 5.626403\end{array}$ 


$\begin{array}{crrr}\mathrm{H} & 8.893989 & 6.319827 & 3.868958 \\ \mathrm{H} & 8.254496 & 7.577648 & 4.951772 \\ \mathrm{H} & 8.110339 & 3.517607 & 4.337081 \\ \mathrm{H} & 8.389128 & 3.705838 & 6.096159 \\ \mathrm{H} & 6.802119 & 3.113230 & 5.498397 \\ \mathrm{H} & 6.242885 & 6.962352 & 6.982233 \\ \mathrm{H} & 5.777674 & 5.257977 & 7.261749 \\ \mathrm{H} & 7.470232 & 5.792143 & 7.579794 \\ \mathrm{H} & 2.120787 & 7.274725 & 1.653327 \\ \mathrm{H} & 3.181056 & 1.802218 & 6.196737 \\ \mathrm{H} & 6.104972 & 0.885559 & 5.160228 \\ \mathrm{H} & 5.314688 & 0.201741 & 6.613170 \\ \mathrm{H} & 5.267348 & -2.374998 & 3.540479 \\ \mathrm{H} & 5.986296 & -0.794338 & 3.101071 \\ \mathrm{H} & 3.100706 & -1.368168 & 3.914490 \\ \mathrm{H} & 4.512921 & 0.179501 & 1.788523 \\ \mathrm{H} & 3.296518 & 0.292281 & -0.178215 \\ \mathrm{H} & 1.167103 & 0.121057 & -1.435780 \\ \mathrm{H} & -0.987712 & -0.173488 & -0.215937 \\ \mathrm{H} & -0.979627 & -0.347125 & 2.270526 \\ \mathrm{H} & 1.135521 & -0.202050 & 3.524251\end{array}$

$\mathrm{X}$

74

scf done: -3347.557760

$\begin{array}{llll}\text { C } & 1.460021 & -1.247513 & 1.852391\end{array}$

$\begin{array}{llll}\text { C } & 0.553592 & -0.715875 & 2.790784\end{array}$

$\begin{array}{llll}\text { C } & 1.047360 & -0.373995 & 4.062448\end{array}$

$\begin{array}{llll}\text { C } & 2.391595 & -0.552163 & 4.375548\end{array}$

$\begin{array}{llll}\text { C } & 3.271573 & -1.088138 & 3.436237\end{array}$

$\begin{array}{llll}\text { C } & 2.798333 & -1.438937 & 2.170983\end{array}$

$\begin{array}{llll}\text { C } & -0.854237 & -0.529170 & 2.387725\end{array}$

$\begin{array}{llll}\text { Pd } & -1.662313 & -2.432572 & 2.386195\end{array}$

$\begin{array}{llll}\mathrm{S} & -1.158903 & -3.310806 & 4.596767\end{array}$

$\begin{array}{llll}\text { Pd } & -2.366236 & -5.076963 & 3.541352\end{array}$

$\begin{array}{llll}\mathrm{P} & -4.189049 & -4.612442 & 4.819424\end{array}$

$\begin{array}{llll}\text { C } & -5.570646 & -3.876407 & 3.893390\end{array}$

$\begin{array}{llll}\text { C } & -1.733317 & 0.389416 & 3.210348\end{array}$

$\begin{array}{llll}\text { C } & -3.188332 & 0.364482 & 2.805655\end{array}$

$\begin{array}{llll}\text { C } & -3.331300 & 1.360941 & 1.685805\end{array}$

$\begin{array}{llll}\mathrm{O} & -2.114404 & 2.073736 & 1.643687\end{array}$

$\begin{array}{llll}\text { C } & -1.421484 & 1.853116 & 2.849757\end{array}$

$\begin{array}{llll}\text { C } & -4.168164 & -0.314210 & 3.401319\end{array}$

$\begin{array}{llll}\text { S } & -2.467044 & -2.202760 & 0.124213\end{array}$

$\begin{array}{llll}\text { Pd } & -3.107447 & -4.441146 & 0.633116\end{array}$

$\begin{array}{llll}\text { S } & -3.796589 & -6.325674 & 1.989389\end{array}$

$\begin{array}{llll}\text { C } & -2.814680 & -7.772503 & 1.457256\end{array}$

$\begin{array}{llll}\text { C } & -1.023949 & -2.355289 & -0.975854\end{array}$

$\begin{array}{llll}\text { C } & 0.592732 & -3.807902 & 4.506750\end{array}$

$\begin{array}{llll}\mathrm{O} & -0.593332 & -5.775625 & 2.614104\end{array}$

$\begin{array}{llll}\text { C } & -0.333137 & -5.630307 & 1.399576\end{array}$

$\begin{array}{llll}\mathrm{O} & -1.046909 & -5.109794 & 0.516743\end{array}$

$\begin{array}{llll}\text { P } & -5.183516 & -3.874091 & -0.102787\end{array}$

$\begin{array}{llll}\text { C } & -5.064808 & -3.671301 & -1.909129\end{array}$

$\begin{array}{llll}\text { C } & -6.491210 & -5.123722 & 0.103174\end{array}$

$\begin{array}{llll}\text { C } & -5.939019 & -2.320730 & 0.472095\end{array}$

$\begin{array}{llll}\text { C } & -4.862110 & -6.149483 & 5.522331\end{array}$

$\begin{array}{llll}\text { C } & -3.989617 & -3.515945 & 6.256157\end{array}$ 


$\begin{array}{rrrr}\mathrm{H} & -3.952598 & -0.983843 & 4.242084 \\ \mathrm{H} & -0.477083 & -1.399387 & -0.951743 \\ \mathrm{H} & -1.375204 & -2.530966 & -2.003764 \\ \mathrm{H} & -0.372290 & -3.181250 & -0.657573 \\ \mathrm{H} & 0.956144 & -3.836565 & 3.471351 \\ \mathrm{H} & 0.719770 & -4.795162 & 4.972181 \\ \mathrm{H} & 1.163559 & -3.058750 & 5.075101 \\ \mathrm{H} & -2.542085 & -7.669874 & 0.397921 \\ \mathrm{H} & -3.435455 & -8.669629 & 1.589661 \\ \mathrm{H} & -1.902864 & -7.862934 & 2.061852 \\ \mathrm{H} & -7.404618 & -4.779047 & -0.408712 \\ \mathrm{H} & -6.161543 & -6.075855 & -0.338542 \\ \mathrm{H} & -6.716274 & -5.296850 & 1.165389 \\ \mathrm{H} & -6.065372 & -3.488204 & -2.335381 \\ \mathrm{H} & -4.404587 & -2.820900 & -2.136775 \\ \mathrm{H} & -4.639173 & -4.581435 & -2.358430 \\ \mathrm{H} & -6.835617 & -2.113972 & -0.134845 \\ \mathrm{H} & -6.233988 & -2.402971 & 1.528100 \\ \mathrm{H} & -5.224452 & -1.490790 & 0.360314 \\ \mathrm{H} & -5.759017 & -5.925494 & 6.122416 \\ \mathrm{H} & -5.122575 & -6.840825 & 4.707192 \\ \mathrm{H} & -4.105816 & -6.626006 & 6.164115 \\ \mathrm{H} & -5.873911 & -4.568373 & 3.092643 \\ \mathrm{H} & -6.426071 & -3.695981 & 4.564673 \\ \mathrm{H} & -5.234892 & -2.924930 & 3.449893 \\ \mathrm{H} & -3.234419 & -3.928738 & 6.941005 \\ \mathrm{H} & -3.654985 & -2.515576 & 5.942086 \\ \mathrm{H} & -4.956918 & -3.430146 & 6.777903 \\ \mathrm{H} & 0.656793 & -6.019734 & 1.065260 \\ \mathrm{H} & -5.213992 & -0.208762 & 3.090511 \\ \mathrm{H} & -3.490521 & 0.865464 & 0.707795 \\ \mathrm{H} & -4.193899 & 2.034679 & 1.859959 \\ \mathrm{H} & -1.780223 & 2.526671 & 3.655822 \\ \mathrm{H} & -0.350198 & 2.050092 & 2.683363 \\ \mathrm{H} & -1.629357 & 0.182053 & 4.288194 \\ \mathrm{H} & -0.914820 & -0.258332 & 1.319722 \\ \mathrm{H} & 1.092879 & -1.507461 & 0.853166 \\ \mathrm{H} & 3.482623 & -1.851155 & 1.424158 \\ \mathrm{H} & 4.327272 & -1.224381 & 3.685995 \\ \mathrm{H} & 2.758095 & -0.264383 & 5.365195 \\ \mathrm{H} & 0.377814 & 0.046697 & 4.818793\end{array}$

\section{TS (X-XI)}

74

scf done: -3347.523158

$\begin{array}{llll}\text { C } & 0.032461 & 0.049061 & 0.023858\end{array}$

$\begin{array}{llll}\text { C } & 0.013878 & -0.009383 & 1.428078\end{array}$

$\begin{array}{llll}\text { C } & 1.243714 & -0.003690 & 2.106656\end{array}$

$\begin{array}{llll}\text { C } & 2.446417 & 0.082512 & 1.412886\end{array}$

$\begin{array}{llll}\text { C } & 2.447308 & 0.153481 & 0.021413\end{array}$

$\begin{array}{llll}\text { C } & 1.235456 & 0.130391 & -0.669158\end{array}$

$\begin{array}{llll}\text { C } & -1.215088 & -0.104528 & 2.231997\end{array}$

Pd $-2.471200 \quad-1.782056 \quad 1.580035$

$\begin{array}{llll}\mathrm{S} & -0.542482 & -3.212658 & 2.422082\end{array}$

C $\quad 0.392911 \quad-3.537222 \quad 0.895038$

$\begin{array}{llll}\text { C } & -2.471089 & 0.459749 & 1.940860\end{array}$

$\begin{array}{llll}\text { C } & -2.824344 & 1.400901 & 0.804619\end{array}$

$\begin{array}{llll}\text { O } & -4.207845 & 1.590576 & 0.917837\end{array}$ 


\begin{tabular}{|c|c|c|c|}
\hline $\mathrm{C}$ & -4.526740 & 1.632083 & 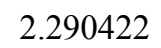 \\
\hline $\mathrm{C}$ & -3.464182 & & \\
\hline $\mathrm{C}$ & -3.409682 & & 906 \\
\hline S & -4.191273 & -3.161927 & 0.472938 \\
\hline $\mathrm{C}$ & -3.340968 & -3.537383 & -1.090457 \\
\hline . & -4.129873 & -5.149580 & 1.754413 \\
\hline 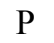 & -5.719759 & -4.226637 & 3.102898 \\
\hline $\mathrm{C}$ & -5.733925 & -2.411655 & .283333 \\
\hline $\mathrm{Pd}$ & -1.559210 & -5.273467 & 4474 \\
\hline$P$ & -1.487956 & -4.564783 & \\
\hline $\mathrm{C}$ & 0.239305 & -4.4 & 0106 \\
\hline $\mathrm{S}$ & -3.421642 & -6.8 & 498 \\
\hline $\mathrm{C}$ & -3.0 & -8.3 & \\
\hline $\mathrm{O}$ & -1.06 & -6.1 & 023 \\
\hline $\mathrm{C}$ & -1.8 & -6.4 & \\
\hline $\mathrm{O}$ & -3.0 & -6.1 & 029 \\
\hline $\mathrm{C}$ & -7.3 & -4.6 & \\
\hline $\mathrm{C}$ & -5.8 & -4.8 & \\
\hline $\mathrm{C}$ & -2.2 & -2.5 & \\
\hline $\mathrm{C}$ & & & \\
\hline $\mathrm{H}$ & -3.5 & -0 & \\
\hline $\mathrm{H}$ & -1.4 & -6.9 & -0. \\
\hline $\mathrm{H}$ & -3.3 & -2.6 & \\
\hline $\mathrm{H}$ & -3.8 & -4.3 & -1 . \\
\hline $\mathrm{H}$ & -2.3 & -3.8 & \\
\hline $\mathrm{H}$ & -0.2 & -3. & \\
\hline $\mathrm{H}$ & 1.1 & -4.3 & \\
\hline $\mathrm{H}$ & 0.8 & -2.5 & \\
\hline $\mathrm{H}$ & -3.4 & -8.2 & \\
\hline $\mathrm{H}$ & -3.6 & -9.1 & 3. \\
\hline $\mathrm{H}$ & -2.0 & -8.5 & \\
\hline $\mathrm{H}$ & -6.7 & -4.7 & \\
\hline $\mathrm{H}$ & -5.9 & -5.9 & \\
\hline $\mathrm{H}$ & -4. & -4 & \\
\hline $\mathrm{H}$ & -8.1 & -4.2 & 46 \\
\hline $\mathrm{H}$ & -7.4 & -4.1 & \\
\hline $\mathrm{H}$ & -7.4 & -5.7 & 82 \\
\hline $\mathrm{H}$ & -6.5 & -2.1 & \\
\hline $\mathrm{H}$ & -4.7 & -2. & \\
\hline $\mathrm{H}$ & -5.8 & -1.9 & \\
\hline $\mathrm{H}$ & -2.1 & -5.2 & \\
\hline $\mathrm{H}$ & -3.3 & -5.8 & \\
\hline $\mathrm{H}$ & -1.73 & -6.6 & \\
\hline $\mathrm{H}$ & -3.2 & -2.9 & 04 \\
\hline $\mathrm{H}$ & -2.0 & -2.6 & \\
\hline $\mathrm{H}$ & -1.72 & -2.1 & \\
\hline $\mathrm{H}$ & 0.7 & -5.3 & 761 \\
\hline $\mathrm{H}$ & 0.760834 & -3.6 & 5.0 \\
\hline $\mathrm{H}$ & 0.2 & -4.0 & 24 \\
\hline $\mathrm{H}$ & -1.023 & -0.2 & 531 \\
\hline $\mathrm{H}$ & -2.586 & 0.006 & \\
\hline $\mathrm{H}$ & -4.188 & 0.9 & 4.979736 \\
\hline $\mathrm{H}$ & -5.543950 & 1.230246 & 2.434095 \\
\hline $\mathrm{H}$ & -4.523599 & 2.675396 & 2.667887 \\
\hline $\mathrm{H}$ & -2.262833 & 2.349380 & 0.966454 \\
\hline $\mathrm{H}$ & -2.599488 & 1.041227 & -0.207106 \\
\hline $\mathrm{H}$ & 1.249275 & -0.064367 & 3.199881 \\
\hline $\mathrm{H}$ & 3.390636 & 0.093242 & 1.964195 \\
\hline $\mathrm{H}$ & 3.39112 & 0.220163 & -0.52634 \\
\hline-3 & 1.226786 & 0.170557 & -1.76165 \\
\hline
\end{tabular}


H $\quad-0.900772 \quad-0.003867 \quad-0.543995$

\section{XI \\ 74}

scf done: -3347.536201

$\begin{array}{llll}\text { Pd } & -1.295887 & 6.958854 & 2.823222\end{array}$

$\begin{array}{llll}\mathrm{Pd} & 0.923261 & 5.787190 & 1.131653\end{array}$

$\begin{array}{llll}\text { Pd } & -1.578083 & 4.007029 & 1.669532\end{array}$

$\begin{array}{llll}\mathrm{H} & -0.549805 & 4.967606 & 2.378803\end{array}$

$\begin{array}{llll}\text { S } & 0.901020 & 7.881862 & 2.372758\end{array}$

$\begin{array}{llll}\mathrm{S} & 0.461227 & 3.568401 & 0.361233\end{array}$

$\begin{array}{llll}\mathrm{S} & -3.034993 & 5.335086 & 3.153902\end{array}$

$\begin{array}{llll}\mathrm{P} & -0.997613 & 6.937871 & 5.078479\end{array}$

$\begin{array}{llll}\mathrm{P} & 2.609448 & 4.848514 & 2.351818\end{array}$

$\begin{array}{llll}\mathrm{O} & -0.304312 & 6.648214 & -0.426386\end{array}$

$\begin{array}{llll}\text { C } & -1.414303 & 7.198005 & -0.251697\end{array}$

$\begin{array}{llll}\text { O } & -2.008633 & 7.416282 & 0.825444\end{array}$

$\begin{array}{llll}\text { C } & 0.037724 & 3.770273 & -1.396283\end{array}$

$\begin{array}{llll}\text { C } & -4.418475 & 5.940127 & 2.137677\end{array}$

$\begin{array}{llll}\text { C } & -3.415155 & 2.753084 & 0.560085\end{array}$

$\begin{array}{llll}\text { C } & -3.345948 & 2.991017 & -0.886476\end{array}$

$\begin{array}{llll}\text { C } & -3.609245 & 4.279849 & -1.380188\end{array}$

$\begin{array}{llll}\mathrm{C} & -2.726083 & 1.815805 & 1.275031\end{array}$

$\begin{array}{llll}\text { C } & -1.657994 & 0.862841 & 0.800998\end{array}$

$\begin{array}{llll}\mathrm{O} & -0.908307 & 0.571439 & 1.953063\end{array}$

$\begin{array}{llll}\text { C } & -1.808019 & 0.509223 & 3.035757\end{array}$

$\begin{array}{llll}\text { C } & -2.931327 & 1.459588 & 2.692312\end{array}$

$\begin{array}{llll}\text { C } & -3.930079 & 1.831589 & 3.500371\end{array}$

$\begin{array}{llll}\text { C } & -3.549690 & 4.548064 & -2.742497\end{array}$

$\begin{array}{llll}\text { C } & -3.246990 & 3.526275 & -3.643495\end{array}$

$\begin{array}{llll}\text { C } & -3.013746 & 2.236165 & -3.170972\end{array}$

$\begin{array}{llll}\text { C } & -3.061173 & 1.969189 & -1.805205\end{array}$

$\begin{array}{llll}\text { C } & 0.689783 & 9.217420 & 1.146859\end{array}$

$\begin{array}{llll}\text { C } & 3.339062 & 5.880635 & 3.663161\end{array}$

$\begin{array}{llll}\text { C } & 2.181390 & 3.304021 & 3.219322\end{array}$

$\begin{array}{llll}\text { C } & 4.020840 & 4.407587 & 1.292887\end{array}$

$\begin{array}{llll}\text { C } & 0.281521 & 8.051509 & 5.740592\end{array}$

$\begin{array}{llll}\text { C } & -2.500080 & 7.426196 & 5.981626\end{array}$

$\begin{array}{llll}\text { C } & -0.556365 & 5.301071 & 5.744076\end{array}$

$\begin{array}{llll}\mathrm{H} & -1.937518 & 7.541827 & -1.176727\end{array}$

$\begin{array}{llll}\mathrm{H} & -0.533489 & 2.885912 & -1.717535\end{array}$

$\mathrm{H} \quad 0.971286 \quad 3.830268 \quad-1.973804$

$\begin{array}{llll}\mathrm{H} & -0.558415 & 4.677259 & -1.557815\end{array}$

$\begin{array}{llll}\mathrm{H} & -4.048017 & 6.372922 & 1.198112\end{array}$

$\begin{array}{llll}\mathrm{H} & -4.963683 & 6.709369 & 2.703427\end{array}$

$\begin{array}{llll}\mathrm{H} & -5.105364 & 5.103355 & 1.934847\end{array}$

$\begin{array}{llll}\mathrm{H} & 0.748621 & 8.816728 & 0.126607\end{array}$

$\begin{array}{llll}\mathrm{H} & 1.498071 & 9.945296 & 1.305472\end{array}$

$\begin{array}{llll}\mathrm{H} & -0.280894 & 9.711282 & 1.293667\end{array}$

$\begin{array}{llll}\mathrm{H} & 4.151382 & 5.320535 & 4.154892\end{array}$

$\begin{array}{llll}\mathrm{H} & 3.738788 & 6.814137 & 3.240250\end{array}$

$\begin{array}{llll}\mathrm{H} & 2.575408 & 6.138874 & 4.413803\end{array}$

$\begin{array}{llll}\mathrm{H} & 4.829451 & 3.972853 & 1.903548\end{array}$

$\begin{array}{llll}\mathrm{H} & 3.693853 & 3.676999 & 0.538364\end{array}$

$\begin{array}{llll}\mathrm{H} & 4.392445 & 5.305957 & 0.777557\end{array}$

$\begin{array}{llll}\mathrm{H} & 3.072690 & 2.925932 & 3.745753\end{array}$

$\begin{array}{llll}\mathrm{H} & 1.377091 & 3.483744 & 3.949795\end{array}$

$\begin{array}{llll}\mathrm{H} & 1.832183 & 2.545360 & 2.501946\end{array}$ 


$\begin{array}{rrrr}\mathrm{H} & 0.310875 & 7.963026 & 6.839091 \\ \mathrm{H} & 1.269087 & 7.807439 & 5.321996 \\ \mathrm{H} & 0.044305 & 9.090081 & 5.464365 \\ \mathrm{H} & 0.454389 & 5.030152 & 5.399796 \\ \mathrm{H} & -0.562800 & 5.324370 & 6.845915 \\ \mathrm{H} & -1.269901 & 4.540509 & 5.388150 \\ \mathrm{H} & -2.823830 & 8.423657 & 5.647540 \\ \mathrm{H} & -3.303099 & 6.704613 & 5.772649 \\ \mathrm{H} & -2.293276 & 7.452711 & 7.064138 \\ \mathrm{H} & -4.191130 & 3.319809 & 1.090831 \\ \mathrm{H} & -4.737119 & 2.487855 & 3.159574 \\ \mathrm{H} & -3.980853 & 1.482009 & 4.535926 \\ \mathrm{H} & -1.277151 & 0.778966 & 3.963911 \\ \mathrm{H} & -2.204089 & -0.520814 & 3.161795 \\ \mathrm{H} & -2.130868 & -0.060025 & 0.393247 \\ \mathrm{H} & -0.994788 & 1.277353 & 0.024685 \\ \mathrm{H} & -3.856474 & 5.079302 & -0.671751 \\ \mathrm{H} & -3.749089 & 5.559425 & -3.108089 \\ \mathrm{H} & -3.209233 & 3.733081 & -4.716386 \\ \mathrm{H} & -2.803071 & 1.425566 & -3.874047 \\ \mathrm{H} & -2.912680 & 0.943907 & -1.454298\end{array}$

\section{$\mathrm{A}-\mathrm{PPh}_{3}$}

120

scf done: -4801.591906

C 20.45769710 .12813220 .062857

C 21.06866810 .35549418 .827450

C 21.68235811 .58931218 .574133

C 21.68998812 .57583819 .551442

C 21.07919312 .34272320 .782315

C 20.46407111 .12193921 .037161

P 21.0801919 .04625917 .561243

C 20.8679419 .96356815 .994061

C 19.65904010 .64684615 .807089

C 19.41747611 .33024614 .623333

C 20.37700511 .33616713 .612083

C 21.58261010 .66947513 .795603

C 21.8320929 .98591814 .984195

Pd 19.578217 7.281913 17.940878

S 18.0701987 .65412216 .183970

C 18.9925067 .16203314 .681856

Pd 18.8016364 .91676219 .529417

S 16.8408443 .93646518 .672321

C 17.3329242 .43433317 .750035

P 19.1812763 .41477121 .293753

C 19.2309471 .71262020 .641711

C 18.3867130 .70406021 .110411

C $18.434942-0.56619520 .539544$

C $19.328589-0.83820119 .509537$

C 20.1712220 .16575519 .034559

C 20.1124941 .43824919 .587759

C 17.9151013 .42631822 .603014

C 16.6796904 .03727822 .375644

C 15.7053884 .03091623 .369453

C 15.9602403 .41581524 .590247

C 17.1911152 .80502624 .823366

C 18.1684212 .81263923 .836835

C 20.7484953 .62697622 .211008 
C 21.7583242 .66278722 .233338 C 22.9469522 .91223622 .916842 C 23.1305474 .11784923 .583499 C 22.1193365 .07742023 .577594 C 20.9355434 .83588722 .894247 S 20.5585136 .42481119 .901667 C 22.0606465 .50253519 .409569 Pd 17.213088 5.693340 17.164272 P 15.4487515 .31371215 .662281 C 14.1395156 .57991115 .673802 C 12.9056976 .32661315 .060109 C 11.9191887 .30394015 .052477 C 12.1523298 .53481815 .663254 C 13.3731228 .78964816 .278365 C 14.3669087 .81532316 .284750 C 22.7823398 .39421617 .511498 C 23.7909168 .86288018 .355733 C 25.0611658 .29200218 .307469 C 25.3331487 .26201917 .413788 C 24.3291766 .78707916 .571152 C 23.0566887.340289 16.629915 C 16.1008055 .26400513 .960138 C 15.6321316 .10823112 .951560 C 16.2030036 .05996311 .681309 C 17.2329895 .16628411 .409326 C 17.7079444 .32366112 .413302 C 17.1547384 .38242713 .685791 C 14.5314673 .74651315 .874495 C 13.8482043 .55949617 .083397 C 13.1648372 .37571617 .324937 C 13.1589361 .36448816 .365383 C 13.8256171 .54805315 .159779 C 14.5091412 .73666914 .910322 H 26.3286016 .82390817 .373600 H 15.1988753 .41454625 .368069 H 24.0609074 .31095024 .114344 H 19.368753 -1.833660 19.071439 H 17.6710885 .12608810 .413869 H 12.6280760 .43413816 .558490 H 11.3745249 .29620115 .661967 H 20.18387911 .86705312 .681752 H 21.08046513 .12054721 .543680 H 12.7204295 .36123214 .587290 H 10.9624697 .10450814 .573416 H 13.5529319 .74875216 .760274 H 15.3254748 .00998116 .765726 H 14.8206486 .80585913 .155265 H 15.8338606 .72221710 .900465 H 18.5180733 .62715412 .205146 H 17.5442073 .74172614 .480632 H 13.8560544 .34586817 .839263 H 12.6372962 .23905218 .266976 H 13.8120010 .76641714 .402463 H 15.0183042 .87381613 .957348 H 16.5233892 .18618317 .051154 H 17.4525711 .61080718 .463429 H 18.2695822 .58665017 .204517 H 22.15519111 .77455617 .608755 H 22.169058 13.532551 19.352001 H 19.98213710 .94215921 .996257 
H 19.9767109 .16957420 .257526 H 23.5872129 .67435019 .053374 H 25.8420038 .66113118 .969738 H 24.5373265 .97694715 .874646 H 22.2618526 .95082415 .989207 H 18.90319610 .63898916 .593483 H 18.47543911 .85780014 .486705 H 22.33991010 .68311613 .013952 H 22.7850709 .47675815 .121309 H 19.6913547 .97158814 .433682 H 18.2791187 .04233113 .858332 H 19.5380636 .22540114 .834202 H 19.1338002 .33981024 .022085 H 17.3905632 .32597225 .780085 H 14.7462874 .51283723 .189663 H 16.4850164 .51824821 .417078 H 17.6891030 .90778321 .921904 H $17.772678-1.34702320 .908703$ H $20.867704-0.04241718 .224412$ H 20.7532012 .23307319 .198270 H 20.1491825 .59176622 .886396 H 22.2560226 .01946624 .105117 H 23.7285762 .15490722 .930462 H 21.6211541 .70980521 .724197 H 22.3087474 .80357220 .219049 H 22.8841926 .21592119 .290028 H 21.9083554 .95711118 .472851

\section{$\mathrm{I}_{-} \mathrm{PPh}_{3}$}

125

scf done: -4991.267062

C 16.7413074 .12453222 .323609

C 17.9484123 .46594022 .570056

C 18.1749962 .88029723 .822889

C 17.1983802 .94930724 .807554

C 15.9998103 .61615224 .557213

C 15.7718184 .20519923 .318402 P 19.2272623 .38285421 .278816

Pd 18.8970544 .85910819 .484554 Pd 19.673055 7.213360 17.885172 P 21.1411639 .01863117 .531890 C 22.8506388 .38675717 .504287 C 23.8417208 .86588518 .363180 C 25.1194128 .31077418 .330935 C 25.415999 7.285482 17.439589 C 24.4288846 .79841816 .584017 C 23.1489187 .33590416 .626358 H 26.4173256 .86010117 .411926 H 15.2437993 .68253425 .337415 C 20.7918683 .59476322 .198819 C 21.7981182 .62701522 .226720 C 22.9875042 .87624722 .909160 C 23.1753814 .08516523 .568464 C 22.1677305 .04862723 .556479 C 20.9832374 .80734622 .874524 H 24.1059184 .27774324 .099118 C 19.2541811 .67121320 .656033 C 18.3942520 .68453421 .141828

C $18.427213-0.59729020 .596433$ 
C $19.320646-0.90145619 .575165$ C 20.1779100 .08150119 .082335 C 20.1346401 .36562719 .609584 H 19.348553 -1.905862 19.157087 S 20.6542446 .36934719 .856950 C 22.1522195 .43676519 .370232 Pd 17.313554 5.633027 17.131745 P 15.5011665 .29702515 .687750 C 16.1103955 .30595213 .970871 C 15.6703436 .22772413 .019118 C 16.2169876 .22044411 .737373 C 17.1973815 .29437211 .398026 C 17.6502854 .38006912 .347859 C 17.1202284 .39488113 .631515 H 17.6155115 .28703510 .393303 S 16.9319323 .87427318 .632198 C 17.4135462 .37681117 .698192 S 18.1572527 .58525716 .118522 C 19.1018027 .07625914 .636035 C 14.5837753 .72854615 .883189 C 13.9092163 .53103517 .095530 C 13.2247152 .34628517 .328688 C 13.2075141 .34645216 .357382 C 13.8642861 .54192315 .148186 C 14.5506142 .73059814 .907158 H 12.6741900 .41614816 .543666 C 14.2093236 .56605215 .833861 C 13.0265626 .45174315 .090236 C 12.0277757 .40603315 .226204 C 12.1926208 .46465916 .118711 C 13.3550108 .57028816 .873690 C 14.3662927 .62361116 .732354 H 11.4029509 .20521716 .231900 C 20.9268049 .92847315 .962655 C 19.71070610 .59665915 .766685 C 19.46729011 .26962614 .577340 C 20.43133411 .27936813 .570382 C 21.64357610 .62731213 .763088 C 21.8955069 .95427314 .957042 H 20.23661411 .80229912 .635921 C 21.07982710 .31853218 .803248 C 21.68891511 .55822218 .568284 C 21.65675112 .54467319 .544912 C 21.00872712 .30605820 .755558 C 20.39608711 .08020520 .991543 C 20.43117010 .08596820 .018487 H 20.97639013 .08438021 .515591 O 16.7990208 .19352019 .374110 C 16.5022397 .31811620 .346815 O 15.4661957 .32417120 .946858 H 12.8878745 .61133414 .408195 H 11.1122977 .31984714 .644029 H 13.4782069 .38336117 .585947 H 15.2709747 .70045217 .336282 H 14.9001546 .95377213 .276585 H 15.8668066 .94040811 .000095 H 18.4249933 .66065612 .089065 H 17.4941503 .69526514 .383415 H 13.9212044 .30994217 .859328 H 12.7032332 .20160018 .272828 
H 13.8396240 .77018314 .381076 H 15.0501772 .87776513 .950725 H 16.5952582 .13444917 .007832 H 17.5385851 .55061118 .407446 H 18.3444722 .53146617 .143581 H 22.188416 11.747844 17.617260 H 22.13226513 .50603819 .360216 H 19.88308510 .89702821 .933504 H 19.9536729 .12257420 .200906 H 23.6193409 .67418019 .058734 H 25.8866808 .68890519 .003842 H 24.6558915 .99106115 .890232 H 22.3685456 .93679415 .973521 H 18.95105010 .58790016 .549599 H 18.52013011 .78586514 .433686 H 22.40426510 .64494412 .984864 H 22.8536479 .45694315 .101774 H 19.8127627 .87845614 .398491 H 18.3996226 .96311313 .802018 H 19.6333376 .13341614 .799909 H 19.1190392 .37257824 .025333 H 17.3752982 .49058825 .778598 H 14.8465444 .74193023 .119911 H 16.5560834 .57548721 .349591 H 17.6973030 .91431221 .946900 H 17.753037 -1.361482 20.978478 H $20.872884-0.15219618 .277904$ H 20.7867212 .14473519 .206331 H 20.1983075 .56490622 .865158 H 22.3072205 .99286524 .079275 H 23.7657562 .11562922 .928560 H 21.6571301 .67089221 .724574 H 22.3942934 .74378420 .186604 H 22.9781756 .14615819 .244759 H 21.9951924 .88386918 .438690 H 17.6351547 .90836218 .940930 H 17.3223706 .58339020 .531688

\section{But-2-yne}

10 scf done: -155.733285

$\begin{array}{rrrr}\mathrm{C} & 0.798283 & 1.942929 & 0.245134 \\ \mathrm{C} & 0.084363 & 0.866742 & 0.910518 \\ \mathrm{C} & -0.510444 & -0.031490 & 1.466903 \\ \mathrm{C} & -1.225039 & -1.106801 & 2.132999 \\ \mathrm{H} & -2.142273 & -0.740731 & 2.622102 \\ \mathrm{H} & -1.523784 & -1.894858 & 1.422998 \\ \mathrm{H} & -0.606130 & -1.583041 & 2.910529 \\ \mathrm{H} & 0.278477 & 2.907475 & 0.363490 \\ \mathrm{H} & 1.814956 & 2.066340 & 0.652133 \\ \mathrm{H} & 0.898039 & 1.754963 & -0.836100\end{array}$

\section{Dimer B}

38

scf done: -2053.346053

$\begin{array}{llll}\text { C } & 10.084475 & 2.080133 & 14.739283\end{array}$ 


$\begin{array}{cccc}\text { P } & 9.595279 & 3.817271 & 14.405966 \\ \mathrm{C} & 9.958029 & 4.623370 & 16.014768 \\ \mathrm{Pd} & 10.323157 & 4.710046 & 12.421216 \\ \mathrm{~S} & 10.110404 & 2.865396 & 10.870808 \\ \mathrm{C} & 11.656694 & 1.937815 & 11.176320 \\ \mathrm{C} & 7.766446 & 3.686835 & 14.442958 \\ \mathrm{~S} & 11.002581 & 6.783624 & 11.355006 \\ \mathrm{C} & 12.814106 & 6.958611 & 11.526120 \\ \mathrm{Pd} & 10.816289 & 4.943988 & 9.803299 \\ \mathrm{P} & 11.416944 & 5.864888 & 7.788811 \\ \mathrm{C} & 13.223140 & 5.836699 & 7.459922 \\ \mathrm{C} & 11.036425 & 7.650267 & 7.600026 \\ \mathrm{C} & 10.740497 & 5.178358 & 6.226921 \\ \mathrm{H} & 13.232083 & 7.418072 & 10.614917 \\ \mathrm{H} & 13.024503 & 7.625164 & 12.376868 \\ \mathrm{H} & 13.305584 & 5.989721 & 11.700942 \\ \mathrm{H} & 11.775059 & 1.187763 & 10.378839 \\ \mathrm{H} & 12.536575 & 2.598227 & 11.177576 \\ \mathrm{H} & 11.599817 & 1.409989 & 12.141283 \\ \mathrm{H} & 11.394084 & 8.039253 & 6.631192 \\ \mathrm{H} & 9.948675 & 7.803231 & 7.676387 \\ \mathrm{H} & 11.510225 & 8.210805 & 8.421417 \\ \mathrm{H} & 11.123985 & 5.727462 & 5.349327 \\ \mathrm{H} & 11.014652 & 4.115528 & 6.143255 \\ \mathrm{H} & 9.641917 & 5.245541 & 6.244828 \\ \mathrm{H} & 13.465339 & 6.352974 & 6.515017 \\ \mathrm{H} & 13.752434 & 6.332463 & 8.289158 \\ \mathrm{H} & 13.574337 & 4.794741 & 7.405331 \\ \mathrm{H} & 9.482114 & 4.081316 & 16.850494 \\ \mathrm{H} & 11.046909 & 4.652031 & 16.174523 \\ \mathrm{H} & 9.589148 & 5.660343 & 15.994668 \\ \mathrm{H} & 7.415008 & 3.203644 & 15.370843 \\ \mathrm{H} & 7.321158 & 4.690268 & 14.361617 \\ \mathrm{H} & 7.437852 & 3.095252 & 13.573598 \\ \mathrm{H} & 9.626851 & 1.705746 & 15.671061 \\ \mathrm{H} & 9.753251 & 1.455854 & 13.893845 \\ \mathrm{H} & 11.180433 & 2.005170 & 14.818214 \\ & & & \end{array}$

Monomer B

34

scf done: -1371.773551

$\begin{array}{llll}\text { C } & -0.579592 & 1.385987 & 1.347998\end{array}$

$\begin{array}{llll}\mathrm{P} & -1.523973 & -0.042534 & 1.962067\end{array}$

$\begin{array}{llll}\mathrm{Pd} & -3.758477 & 0.267138 & 1.554054\end{array}$

$\begin{array}{llll}\mathrm{O} & -3.436625 & 2.303053 & 0.715769\end{array}$

$\begin{array}{llll}\text { C } & -3.988611 & 2.812595 & -0.236870\end{array}$

$\begin{array}{llll}\mathrm{O} & -3.661513 & 4.012588 & -0.634885\end{array}$

$\begin{array}{llll}\text { C } & -0.745726 & -1.489153 & 1.189709\end{array}$

$\begin{array}{llll}\text { C } & -1.130049 & -0.149389 & 3.731377\end{array}$

$\begin{array}{llll}\text { S } & -3.939833 & -1.864314 & 2.419129\end{array}$

$\begin{array}{llll}\text { C } & -4.894638 & -1.523667 & 3.925576\end{array}$

$\begin{array}{llll}\text { C } & -6.045654 & 0.154848 & 0.867450\end{array}$

$\begin{array}{llll}\text { C } & -6.264455 & -0.780071 & -0.225944\end{array}$

$\begin{array}{llll}\text { C } & -6.016497 & 0.949744 & 1.803150\end{array}$

$\begin{array}{llll}\text { C } & -6.143609 & 1.910806 & 2.889492\end{array}$

$\begin{array}{llll}\mathrm{H} & -4.354244 & -0.848665 & 4.609162\end{array}$

$\begin{array}{llll}\mathrm{H} & -5.025612 & -2.491208 & 4.435909\end{array}$

H $\quad-5.890173 \quad-1.111552 \quad 3.699374$ 


$\begin{array}{lrrr}\mathrm{H} & -5.663261 & -0.523146 & -1.113056 \\ \mathrm{H} & -5.990744 & -1.800934 & 0.084240 \\ \mathrm{H} & -7.325669 & -0.778773 & -0.520118 \\ \mathrm{H} & -5.810485 & 1.481201 & 3.848179 \\ \mathrm{H} & -5.546184 & 2.817193 & 2.701776 \\ \mathrm{H} & -7.195842 & 2.215211 & 3.005047 \\ \mathrm{H} & -4.789136 & 2.300649 & -0.814129 \\ \mathrm{H} & -4.182510 & 4.294313 & -1.404023 \\ \mathrm{H} & -1.548559 & 0.719614 & 4.261574 \\ \mathrm{H} & -1.568086 & -1.070930 & 4.142048 \\ \mathrm{H} & -0.036456 & -0.164813 & 3.867404 \\ \mathrm{H} & -0.980883 & 2.314234 & 1.780122 \\ \mathrm{H} & 0.482824 & 1.274185 & 1.619112 \\ \mathrm{H} & -0.673152 & 1.448224 & 0.253617 \\ \mathrm{H} & -1.218875 & -2.408401 & 1.563973 \\ \mathrm{H} & -0.864050 & -1.439467 & 0.097135 \\ \mathrm{H} & 0.327969 & -1.500155 & 1.438262\end{array}$

Dimer C

43

scf done: -2491.099005

$\begin{array}{llll}\text { C } & 9.300181 & 2.099876 & 14.105465\end{array}$

$\begin{array}{llll}\text { P } & 9.527407 & 3.820473 & 14.675847\end{array}$

$\begin{array}{llll}\mathrm{Pd} & 10.370142 & 5.235280 & 13.077737\end{array}$

S $\quad 11.136163 \quad 6.913355 \quad 11.424160$

Pd $11.287750 \quad 5.306848 \quad 9.686530$

$\begin{array}{llll}\mathrm{P} & 11.359825 & 3.767530 & 7.914957\end{array}$

$\begin{array}{llll}\text { C } & 13.044854 & 3.521855 & 7.262100\end{array}$

$\begin{array}{llll}\text { C } & 7.876427 & 4.297522 & 15.271974\end{array}$

$\begin{array}{llll}\text { C } & 10.546486 & 3.656175 & 16.173184\end{array}$

$\begin{array}{llll}\mathrm{S} & 10.781511 & 3.676556 & 11.261300\end{array}$

C $12.453432 \quad 3.092438 \quad 11.694143$

$\begin{array}{llll}\text { S } & 10.201132 & 6.864900 & 14.756020\end{array}$

$\begin{array}{llll}\text { C } & 8.813052 & 7.854181 & 14.118289\end{array}$

$\begin{array}{llll}\text { C } & 12.857287 & 7.144800 & 11.979582\end{array}$

$\begin{array}{llll}\text { C } & 10.392080 & 4.352743 & 6.484709\end{array}$

C $\quad 10.740911 \quad 2.083057 \quad 8.216779$

$\begin{array}{llll}\mathrm{H} & 13.391335 & 7.803199 & 11.280227\end{array}$

$\begin{array}{llll}\mathrm{H} & 12.824970 & 7.608059 & 12.977058\end{array}$

$\mathrm{H} \quad 13.385366 \quad 6.181002 \quad 12.052131$

H $\quad 12.903563 \quad 2.562874 \quad 10.840676$

$\begin{array}{llll}\mathrm{H} & 13.103984 & 3.920915 & 12.008768\end{array}$

H $\quad 12.347822 \quad 2.388539 \quad 12.534770$

$\mathrm{H} \quad 8.673631 \quad 8.700254 \quad 14.809664$

H $\quad 9.020976 \quad 8.253582 \quad 13.113616$

$\begin{array}{llll}\mathrm{H} & 7.875816 & 7.274208 & 14.094784\end{array}$

$\mathrm{H} \quad 13.021883 \quad 2.843460 \quad 6.393386$

$\begin{array}{llll}\mathrm{H} & 13.472703 & 4.486583 & 6.950549\end{array}$

H $\quad 13.691133 \quad 3.087999 \quad 8.040684$

H $\quad 10.484463 \quad 3.640479 \quad 5.648179$

$\begin{array}{llll}\mathrm{H} & 9.331869 & 4.443901 & 6.764779\end{array}$

$\mathrm{H} \quad 10.755486 \quad 5.338856 \quad 6.158235$

$\begin{array}{llll}\mathrm{H} & 10.799268 & 1.491008 & 7.289039\end{array}$

H $\quad 11.341663 \quad 1.589637 \quad 8.996016$

H $\quad 9.696083 \quad 2.124760 \quad 8.559285$

H $\quad 10.066468 \quad 2.958199 \quad 16.878520$

H $\quad 11.543596 \quad 3.274531 \quad 15.905583$

H $\quad 10.655591 \quad 4.642982 \quad 16.646996$ 


$\begin{array}{cccc}\mathrm{H} & 7.504755 & 3.552490 & 15.994353 \\ \mathrm{H} & 7.951343 & 5.282915 & 15.755249 \\ \mathrm{H} & 7.177623 & 4.364475 & 14.423873 \\ \mathrm{H} & 8.871569 & 1.485608 & 14.914205 \\ \mathrm{H} & 8.621934 & 2.079040 & 13.238833 \\ \mathrm{H} & 10.266534 & 1.666164 & 13.805932\end{array}$

\section{Monomer C}

29

scf done: -934.022716

$\begin{array}{llll}\text { C } & -1.205693 & -1.186406 & 3.306230\end{array}$

$\begin{array}{llll}\mathrm{P} & -1.719502 & 0.209691 & 2.243935\end{array}$

$\begin{array}{llll}\text { C } & -2.448557 & 1.402559 & 3.408444\end{array}$

$\begin{array}{llll}\text { Pd } & -3.103073 & -0.334529 & 0.519867\end{array}$

$\begin{array}{llll}\text { C } & -4.241003 & -1.119447 & -1.363343\end{array}$

$\begin{array}{llll}\text { C } & -3.379623 & -1.251937 & -2.528126\end{array}$

$\begin{array}{llll}\text { C } & -0.126974 & 0.955910 & 1.764653\end{array}$

$\begin{array}{llll}\text { O } & -2.449615 & 1.131087 & -0.920838\end{array}$

$\begin{array}{llll}\text { C } & -2.300862 & 2.341096 & -0.560384\end{array}$

$\begin{array}{llll}\mathrm{O} & -2.380085 & 2.809494 & 0.571770\end{array}$

$\begin{array}{llll}\text { C } & -5.089434 & -1.018930 & -0.478940\end{array}$

$\begin{array}{llll}\text { C } & -6.263036 & -1.007611 & 0.386510\end{array}$

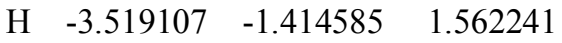

$\mathrm{H} \quad-2.823410-0.313122 \quad-2.676567$

$\begin{array}{llll}\mathrm{H} & -2.649951 & -2.066667 & -2.397822\end{array}$

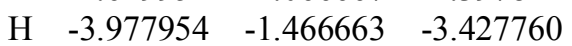

$\begin{array}{llll}\mathrm{H} & -6.396639 & -0.030493 & 0.875379\end{array}$

$\begin{array}{llll}\mathrm{H} & -7.170026 & -1.222367 & -0.200944\end{array}$

$\begin{array}{llll}\mathrm{H} & -6.177133 & -1.767371 & 1.179074\end{array}$

$\begin{array}{llll}\mathrm{H} & -2.058795 & 3.028632 & -1.418485\end{array}$

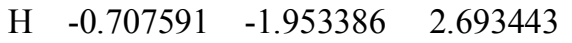

$\begin{array}{llll}\mathrm{H} & -0.511591 & -0.842349 & 4.091076\end{array}$

$\begin{array}{llll}\mathrm{H} & -2.092157 & -1.641626 & 3.773390\end{array}$

$\begin{array}{llll}\mathrm{H} & -2.679744 & 2.309219 & 2.829983\end{array}$

$\begin{array}{llll}\mathrm{H} & -3.374439 & 0.987892 & 3.834648\end{array}$

$\begin{array}{llll}\mathrm{H} & -1.736847 & 1.629532 & 4.219538\end{array}$

$\begin{array}{llll}\mathrm{H} & 0.506329 & 1.088017 & 2.657890\end{array}$

$\begin{array}{llll}\mathrm{H} & 0.392217 & 0.306339 & 1.043870\end{array}$

$\begin{array}{llll}\mathrm{H} & -0.331854 & 1.930909 & 1.300097\end{array}$

\section{Dimer D}

53

scf done: -2646.876526
$\begin{array}{llll}\text { C } & 9.339852 & 2.058311 & 14.125012\end{array}$
$\begin{array}{llll}\text { P } & 9.633118 & 3.760825 & 14.721808\end{array}$
$\begin{array}{llll}C & 10.959741 & 3.576126 & 15.956117\end{array}$
$\begin{array}{llll}\text { Pd } & 10.094299 & 5.296861 & 13.074790\end{array}$
$\begin{array}{llll}\text { S } & 10.074753 & 6.879260 & 14.812473\end{array}$
$\begin{array}{llll}\text { C } & 8.500074 & 7.722481 & 14.462158\end{array}$
$\begin{array}{llll}\text { C } & 8.133533 & 4.113403 & 15.692613\end{array}$
$\begin{array}{llll}\text { S } & 10.424685 & 7.004570 & 11.359557\end{array}$
$\begin{array}{llll}\text { C } & 12.030147 & 7.728878 & 11.824286\end{array}$
$\begin{array}{llll}\text { S } & 10.387368 & 3.764280 & 11.195413\end{array}$
$\begin{array}{llll}\text { C } & 12.085507 & 3.216985 & 11.605670\end{array}$
$\begin{array}{llll}\text { Pd } & 10.969069 & 5.440499 & 9.615642\end{array}$
$\begin{array}{llll}\text { P } & 11.216558 & 3.833580 & 7.894625\end{array}$ 


$\begin{array}{cccc}\mathrm{C} & 10.574474 & 2.165335 & 8.242974 \\ \mathrm{C} & 12.922347 & 3.541909 & 7.311689 \\ \mathrm{C} & 10.328936 & 4.313410 & 6.374045 \\ \mathrm{H} & 12.348341 & 8.450416 & 11.056653 \\ \mathrm{H} & 11.888465 & 8.244914 & 12.785791 \\ \mathrm{H} & 12.796693 & 6.948596 & 11.958477 \\ \mathrm{H} & 12.510804 & 2.632054 & 10.774915 \\ \mathrm{H} & 12.737687 & 4.076221 & 11.822728 \\ \mathrm{H} & 12.038099 & 2.576144 & 12.500432 \\ \mathrm{H} & 8.401890 & 8.554098 & 15.177818 \\ \mathrm{H} & 8.476431 & 8.134639 & 13.441264 \\ \mathrm{H} & 7.638326 & 7.048748 & 14.600293 \\ \mathrm{H} & 12.931536 & 2.715592 & 6.582087 \\ \mathrm{H} & 13.308640 & 4.447325 & 6.818080 \\ \mathrm{H} & 13.582331 & 3.284975 & 8.154999 \\ \mathrm{H} & 10.491253 & 3.555351 & 5.590357 \\ \mathrm{H} & 9.249618 & 4.388794 & 6.577972 \\ \mathrm{H} & 10.695130 & 5.287331 & 6.013336 \\ \mathrm{H} & 10.714935 & 1.523676 & 7.358137 \\ \mathrm{H} & 11.094857 & 1.717751 & 9.102923 \\ \mathrm{H} & 9.503344 & 2.226567 & 8.487032 \\ \mathrm{H} & 10.674439 & 2.835122 & 16.720523 \\ \mathrm{H} & 11.885731 & 3.246349 & 15.459483 \\ \mathrm{H} & 11.140010 & 4.550610 & 16.434646 \\ \mathrm{H} & 7.966719 & 3.317657 & 16.436861 \\ \mathrm{H} & 8.259718 & 5.081339 & 16.200117 \\ \mathrm{H} & 7.262992 & 4.170777 & 15.021015 \\ \mathrm{H} & 9.136910 & 1.387889 & 14.976506 \\ \mathrm{H} & 8.472895 & 2.051019 & 13.446434 \\ \mathrm{H} & 10.213659 & 1.679423 & 13.573039 \\ \mathrm{C} & 12.210331 & 6.983732 & 8.428604 \\ \mathrm{C} & 11.035397 & 7.244169 & 8.160864 \\ \mathrm{C} & 9.731066 & 7.756551 & 7.771539 \\ \mathrm{C} & 13.648394 & 6.907393 & 8.650549 \\ \mathrm{H} & 14.163145 & 7.658344 & 8.030538 \\ \mathrm{H} & 13.894686 & 7.112717 & 9.704529 \\ \mathrm{H} & 14.060869 & 5.918927 & 8.396702 \\ \mathrm{H} & 9.096848 & 6.978665 & 7.319081 \\ \mathrm{H} & 9.200904 & 8.156873 & 8.650418 \\ \mathrm{H} & 9.849924 & 8.569844 & 7.038418\end{array}$

\section{Monomer D}

19

scf done: -778.258017

$\begin{array}{cccc}\mathrm{C} & -1.013507 & -1.024320 & 3.594933 \\ \mathrm{P} & -1.741872 & 0.152766 & 2.412837 \\ \mathrm{C} & -0.440353 & 0.416329 & 1.172028 \\ \mathrm{C} & -1.862661 & 1.723391 & 3.312621 \\ \mathrm{Pd} & -3.650314 & -0.365000 & 1.445762 \\ \mathrm{O} & -3.780854 & 0.577840 & -0.435981 \\ \mathrm{C} & -3.331487 & 1.759781 & -0.593804 \\ \mathrm{O} & -2.703820 & 2.440465 & 0.211792 \\ \mathrm{H} & -3.674500 & -1.090083 & 2.829336 \\ \mathrm{H} & -3.554031 & 2.184104 & -1.611109 \\ \mathrm{H} & -0.899034 & -2.009827 & 3.119096 \\ \mathrm{H} & -0.026579 & -0.662209 & 3.927392 \\ \mathrm{H} & -1.679003 & -1.132952 & 4.464330 \\ \mathrm{H} & -2.200298 & 2.477961 & 2.586629\end{array}$




$\begin{array}{crrc}\mathrm{H} & -2.595060 & 1.636472 & 4.128412 \\ \mathrm{H} & -0.876294 & 1.997518 & 3.722857 \\ \mathrm{H} & 0.498471 & 0.692622 & 1.681282 \\ \mathrm{H} & -0.284681 & -0.501103 & 0.585388 \\ \mathrm{H} & -0.771018 & 1.232824 & 0.511670\end{array}$


Comprehensive table in A. U. XYZ

\begin{tabular}{|c|c|c|c|}
\hline Def2-SVP gas & $\mathrm{H}$ (Hartrees) & $\mathrm{S}\left(\mathrm{cal} / \mathrm{K}^{*} \mathrm{~mol}\right)$ & Imaginary freq $(1 / \mathrm{cm})$ \\
\hline $\mathrm{HCOOH}$ & -189.485318 & 59.445 & \\
\hline $\mathrm{PMe}_{3}$ & -460.665717 & 77.298 & \\
\hline Enyne 1 & -538.612238 & 110.101 & \\
\hline Diene 3 & -538.671063 & 104.733 & \\
\hline A & -3079.450937 & 242.41 & \\
\hline I & -3268.952014 & 252.22 & \\
\hline TS (I-IV) & -3268.922247 & 245.348 & -1122.2886 \\
\hline IV & -3268.927456 & 249.938 & \\
\hline $\mathbf{v}$ & -3268.928131 & 254.433 & \\
\hline VI & -3346.860462 & 267.864 & \\
\hline VII & -3346.859814 & 266.219 & \\
\hline TS (VII-VIII) & -3346.845818 & 264.784 & -589.627 \\
\hline VIII & -3346.894389 & 264.158 & \\
\hline TS (VIII-IX) & -3346.888106 & 257.015 & -284.5494 \\
\hline IX & -3346.9261 & 256.753 & \\
\hline$x$ & -3346.930069 & 261.608 & \\
\hline TS (X-XI) & -3346.901243 & 259.952 & -305.9609 \\
\hline $\mathbf{X I}$ & -3346.915025 & 262.235 & \\
\hline \multicolumn{4}{|l|}{ Lacvp* gas } \\
\hline $\mathrm{HCOOH}$ & -189.619118 & 59.444 & \\
\hline $\mathrm{PMe}_{3}$ & -460.836703 & 77.130 & \\
\hline Enyne 1 & -538.990343 & 110.074 & \\
\hline Diene 3 & -3344.700641 & 286.521 & \\
\hline A & -3076.873327 & 236.699 & \\
\hline I & -3266.510690 & 251.966 & \\
\hline II & -3344.668093 & 276.279 & \\
\hline TS (II-III) & -3344.605827 & 267.474 & -691.9568 \\
\hline III & -3344.629136 & 271.735 & \\
\hline TS (I-IV) & -3266.477878 & 249.269 & -898.0264 \\
\hline IV & -3266.478994 & 251.647 & \\
\hline $\mathbf{v}$ & -3266.481479 & 242.352 & \\
\hline VII & -3344.612853 & 277.837 & \\
\hline TS (VII-VIII) & -3344.606167 & 265.268 & -627.498 \\
\hline VIII & -3344.659081 & 260.11 & \\
\hline TS (VIII-IX) & -3344.653541 & 257.926 & -302.3687 \\
\hline IX & -3344.693056 & 260.912 & \\
\hline$x$ & -3344.696934 & 263.003 & \\
\hline TS (X-XI) & -3344.668010 & 265.126 & -667.171 \\
\hline $\mathbf{X I}$ & -3344.679811 & 262.583 & \\
\hline
\end{tabular}




\section{Lacup* toluene}

HCOOH
PMe 3
Enyne 1
Diene 3
A
I
TS (I-IV)
IV
V
VI
VII
TS (VII-VIII)
VIII
TS (VIII-IX)
IX
X
TS (X-XI)
XI

$\begin{array}{lcc}-189.62448 & 59.39 & \\ -460.837991 & 77.106 & \\ -538.993518 & 109.343 & \\ -539.053099 & 104.556 & \\ -3076.904891 & 234.524 & \\ -3266.546544 & 244.641 & -1025.4245 \\ -3266.510874 & 240.958 & \\ -3266.513946 & 243.268 & \\ -3266.515372 & 243.696 & \\ -3344.660494 & 271.184 & \\ -3344.657858 & 266.311 & -600.5839 \\ -3344.641033 & 266.236 & -302.3837 \\ -3344.693951 & 259.466 & \\ -3344.688446 & 253.489 & -346.1157 \\ -3344.727678 & 256.199 & \\ -3344.731576 & 257.153 & \\ -3344.703073 & 267.375 & \\ -3344.713065 & 261.441 & \end{array}$

Def2-SVP toluene

$\begin{array}{lcc}\text { HCOOH } & -189.490525 & 59.389 \\ \text { PMe }_{3} & -460.666843 & 77.285 \\ \text { Enyne 1 } & -538.615394 & 109.968 \\ \text { Diene 3 } & -538.673614 & 104.672 \\ \text { A } & -3079.482353 & 236.494 \\ \text { A-PPh } & -4800.64388 & 370.241 \\ \text { I } & -3268.988824 & 248.9 \\ \text { I-PPh } 3 & -4990.284354 & 390.371 \\ \text { But-2-yne } & -155.650087 & 67.895 \\ \text { Dimer B } & -2053.042624 & 182.334 \\ \text { Monomer B } & -1371.499508 & 159.995 \\ \text { Dimer C } & -2490.754259 & 192.115 \\ \text { Monomer C } & -933.792938 & 142.464 \\ \text { Dimer D } & -2646.444779 & 214.298 \\ \text { Monomer D } & -778.113432 & 109.985 \\ \text { II } & -3346.931341 & 270.95 \\ \text { TS (II-III) } & -3346.873489 & 263.415 \\ \text { III } & -3346.934629 & 263.273 \\ \text { TS (I-IV) } & -3268.955183 & 240.237 \\ \text { IV } & -3268.963006 & 248.867 \\ \text { V } & -3268.962483 & 241.629\end{array}$




$\begin{array}{lccc}\text { VI } & -3346.893645 & 266.044 & \\ \text { VII } & -3346.89355 & 265.885 & \\ \text { TS (VIII-VIII) } & -3346.880319 & 263.798 & -562.7631 \\ \text { VIII } & -3346.92913 & 261.827 & \\ \text { TS (VIII-IX) } & -3346.922626 & 254.259 & -287.7207 \\ \text { IX } & -3346.96112 & 256.333 & \\ \text { X } & -3346.965618 & 262.068 & -310.4793 \\ \text { TS (X-XI) } & -3346.935869 & 259.358 & \\ \text { XI } & -3346.948574 & 263.761 & \end{array}$

